Aus dem Zentrum der Psychiatrie

des Klinikums der Johann Wolfgang Goethe-Universität

Frankfurt am Main

Klinik für Psychiatrie und Psychotherapie

des Kindes und Jugendalters

Direktor Prof. Dr. med. F. Poustka

\title{
Kognitive und emotionale Entwicklung von Kindern mit pränataler Opiatexposition in Abhängigkeit der postnatalen Betreuungsbedingungen
}

\author{
Dissertation \\ zur Erlangung des Doktorgrades der theoretischen Medizin \\ des Fachbereiches Medizin der \\ Johann Wolfgang Goethe-Universität Frankfurt am Main
}

\author{
Vorgelegt von \\ Silke Schiemann \\ aus Wien, Österreich
}

Frankfurt am Main, 2006 
Dekan: $\quad$ Prof. Dr. J. Pfeilschifter

Referent: Prof. Dr. F. Poustka

Koreferent: Prof. Dr. K. Bauer

Tag der mündlichen Prüfung: 26.4.2007 
Für Ben, Anna und Kai 
1 EINLEITUNG 4

2 FORSCHUNGSANSÄTZE

3 DIE LEBENSSITUATION VON KINDERN SÜCHTIGER ELTERN RISIKOFAKTOREN

3.1 Medizinische Risikofaktoren

3.1.1 Spezifische Effekte der Kokain- bzw. Opiatexposition

3.1.2 Das Entzugssyndrom

3.2 Psychosoziale Risikofaktoren

4 DROGENABHÄNGIGE UND ELTERNSCHAFT

4.1 Einstellungen und Werthaltungen der Eltern

4.2 Qualität der Eltern-Kind-Interaktion

4.4 Fremdplatzierungen - Unterbrechung der Beziehung zu den Eltern 17

4.4.1 Risiken für Fremdunterbringung

4.4.2 Substitution und Fremdplatzierung

5.1 Kognitive Entwicklung $\quad 20$

5.1.1 Effekte von Kokain $\quad 21$

$\begin{array}{ll}5.1 .2 \text { Effekte der Opiate } & 24\end{array}$

5.2 Sozial-emotionale Entwicklung $\quad 26$

5.3 Entwicklung unter dem Aspekt unterschiedlicher Erziehungsbedingungen 29

5.4 Zusammenfassung bisheriger Forschungsergebnisse 33

6 HINTERGRUND UND ZIEL DER AKTUELLEN UNTERSUCHUNG 35

$\begin{array}{ll}7 \text { HYPOTHESEN UND FRAGEN } & 37\end{array}$

8 METHODE $\quad 39$

8.1 Stichprobenrekrutierung $\quad 39$

8.1.1 Die Stichprobe der aktuellen Untersuchung 39

8.1.2 Schwierigkeiten bei der Rekrutierung 39

8.2 Untersuchungsteilnehmer 41

8.2.1 Beschreibung der Ausgangsstichprobe $\quad 41$

8.2.1.1 Substanzeinnahme 41

8.2.1.2 Substitution $\quad 42$

8.2.1.3 HIV-Status 42 
8.2.1.4 Neonatologische Daten der Kinder

8.2.1.5 Medizinische Komplikationen der Kinder in der Kontrollgruppe 43

8.2.2 Betreuungssituation

8.2.2.1 Betreuungsstatus ursprünglich und aktuell 43

8.2.2.2 Betreuungswechsel

8.2.3 Gruppeneinteilung nach dem Betreuungsstatus (EG1, EG2, EG3, KG1)

8.2.4 EG1, EG2, EG3, KG1 - Charakteristika der leiblichen Mütter 50

8.2.4.1 Alter bei Entbindung 51

8.2.4.2 Parität

8.2.4.3 Sozio-ökonomischer Status 51

8.2.4.4 Substitution bei Geburt 52

8.2.4.5 Nationalität der Mütter 52

8.2.4.6 Zuwendungsverhalten und Kooperation zum Zeitpunkt der Geburt 53

8.2.5 EG1, EG2, EG3, KG1 - Charakteristika der aktuellen Bezugspersonen 54

8.2.5.1 Schulbildung, Lebensunterhalt und Beziehungsstatus $\quad 54$

8.2.5.2 Aktuelle körperliche Verfassung der Bezugspersonen $\quad 55$

8.2.5.3 Aktueller Alkohol- und Drogenkonsum 56

8.2.6 EG1, EG2, EG3, KG1 - Charakteristika der Kinder 57

8.2.6.1 Geschlecht, Altersverteilung und Schulbesuch $\quad 57$

8.2.6.2 HIV-Status der Kinder 58

8.3 Untersuchungsinstrumente $\quad 59$

8.3.1 Der Datenerfassungsbogen $\quad 59$

8.3.2 Interviews und Testverfahren bei Kindern $\quad 59$

8.3.3 Interviews und Testverfahren bei Müttern/Bezugspersonen $\quad 61$

$\begin{array}{ll}8.4 \text { Untersuchungsdurchführung } & 61\end{array}$

$\begin{array}{ll}8.5 \text { Untersuchungsdesign } & 62\end{array}$

8.5.1 Kriterien 63

8.5.2 Einflussfaktoren $\quad 63$

$\begin{array}{ll}\text { 8.5.3 Versuchsplan } & 65\end{array}$

8.5.4 Statistische Verfahren 66

\section{ERGEBNISSE $\quad 67$}

$\begin{array}{ll}9.1 \text { Prädiktoren/Einflussfaktoren } & 67\end{array}$

9.1.1. Somatische Variablen der Kinder $\quad 67$

9.1.2 Psychosoziale Faktoren 67

9.1.2.1 Zusammenhang von Gruppenzugehörigkeit und psychosozialer Belastung (Hypothese I)

9.1.3 Einflussfaktoren der Mütter/Bezugspersonen $\quad 70$

9.1.3.1 Psychopathologie der Mütter/Bezugspersonen $\quad 70$

9.1.3.2 Schulbildung der Bezugspersonen 71

9.1.4 Suchtspezifische Einflussfaktoren $\quad 72$

9.2 Korrelationsstatistische Auswertung 72

9.2.1 Korrelationskoeffizienten für EG1, EG2, EG3

9.2.1.1 Zusammenhang NAS und kognitive Entwicklung (Hypothese II)

9.2.2 Korrelationskoeffizienten für KG1 76

9.2.3 Korrelationen für mütterliche Psychopathologie und Schulbildung 77

9.2.4 Auswertung für die Hypothesenüberprüfung III und IV 77

9.3 Kognitive Entwicklung $\quad 80$

9.3.1 Gruppeneffekte $\quad 80$

9.3.2 Verteilung innerhalb der Gruppen 82

9.4 Psychopathologie $\quad 84$

9.4.1 Gruppeneffekte $\quad 84$ 
9.4.2 Verteilung der klinisch auffälligen Werte $\quad 85$

9.4.3 ICD 10 Diagnosen $\quad 86$

9.4.4 Missbrauch psychotroper Substanzen $\quad 87$

$\begin{array}{lr}\text { 9.5 Globales psychosoziales Funktionsniveau } & \mathbf{8 8}\end{array}$

$\begin{array}{ll}\text { 9.5.1 Gruppeneffekte } & 88\end{array}$

9.5.2 Verteilung der GAF-Werte $\quad 88$

$\begin{array}{lr}9.6 \text { Regressionsanalyse } & 90\end{array}$

10 DISKUSSION

$\begin{array}{lr}10.1 \text { Betreuungssituation } & 93\end{array}$

10.2 Biologische Vulnerabilität und psychosoziale Belastung 94

\begin{tabular}{l} 
10.3 Die Entwicklung der Kinder \\
\hline
\end{tabular}

11 ZUSAMMENFASSUNG $\quad 104$

12 SUMMARY 107

13 LITERATURVERZEICHNIS $\quad 109$

14 ANHANG $\quad 120$

Anhang A - Gruppeneinteilung nach dem Betreuungsstatus $\quad 121$

Anhang B - Achse VI: Stufen der Ausprägung 123

Anhang C - Danksagung 124

$\begin{array}{ll}\text { Anhang D - Curriculum vitae } & 125\end{array}$

Anhang E - Ehrenwörtliche Erklärung $\quad 126$ 


\section{Einleitung}

Während die Gefahren des Substanzkonsums im Jugendalter und die „lauernden“ Versuchungen auf dem Schulhof (z.B. Focus 2004, Titelthema „Sucht an der Schule“) derzeit wieder große Aufmerksamkeit in den Medien genießen, stellen die Kinder drogenabhängiger Eltern eine in der Forschung wenig beachtete Gruppe dar. Im Drogen- und Suchtbericht 2005 des deutschen Bundesministeriums für Gesundheit werden aktuelle Zahlen von schätzungsweise 120.000 bis 150.000 Opiatabhängigen und ca. 440.000 Kokainkonsumenten in Deutschland angegeben. Ein großer Teil des Berichtes widmet sich zu Recht der Primärund Sekundärprävention bei Kindern und Jugendlichen, Informationen über die Situation der Kinder substanzabhängiger Eltern sucht man allerdings vergeblich. Dabei wird geschätzt, dass ca. 1/3 der opiatabhängigen Eltern selbst Kinder haben, und zwar durchschnittlich 1,5. Bei den substituierten Drogenabhängigen geht man davon aus, dass ca. 50\% Eltern sind (Klein, 2003). Trotz dieser keineswegs geringen Zahlen sind auch in der internationalen Forschung Studien über die Lebenssituation und Entwicklung dieser Kinder rar, vor allem was die emotionale und soziale Entwicklung anbelangt, und die Ergebnisse sind wenig einheitlich. Kindern von Eltern mit anderen Abhängigkeiten kommt auch mehr Aufmerksamkeit zu als Kindern opiatabhängiger Eltern, und während die Kinder Alkoholabhängiger in den USA spätestens seit den sechziger Jahren in der Forschung Beachtung fanden, erreichte die in den späten siebziger Jahren einsetzende Forschung bezüglich der Kinder aus drogenbelasteten Familien, und hier vor allem in Bezug auf kokainsüchtige Eltern, nie den gleichen Umfang. In Deutschland rückte diese Thematik erst in den letzten Jahren mehr ins Blickfeld.

Wie Hogan (1998) in einem Übersichtsartikel aufführt, gibt es aber mehrere Argumente dafür, die Kinder drogenabhängiger Mütter als separate Gruppe zu betrachten: Im Vergleich zu Alkoholabhängigen sind Drogenabhängige einem größeren Armutsrisiko ausgesetzt und die damit verbundenen Gesundheitsrisiken unterscheiden sich deutlich von den mit Alkoholkonsum assoziierten körperlichen Problemen. Anders als beim Alkohol ist der Gebrauch von Opium oder Kokain an sich illegal und das Risiko für weitere kriminelle Handlungen (Beschaffungskriminalität) und darauf folgende Inhaftierungen der Eltern ist erhöht. Die Sozialkontakte sind oft auf die „Szene“ beschränkt und die Werthaltungen der Eltern in Bezug auf die Gesellschaft sind oft geprägt von Misstrauen und Feindseligkeit. Depressive und antisoziale Symptome treten bei Drogenabhängigen verstärkt auf. Die 
Reaktion der Gesellschaft auf Drogenabhängige ist fast immer pauschal negativ und die Fähigkeit zu einer verantwortungsbewussten Elternschaft wird den Betroffenen meist nicht zugestanden (Klein, 2003).

Die aktuelle und bisherige Forschung geht davon aus, dass der elterliche Drogenkonsum die kindliche psychosoziale Entwicklung in mindestens drei Bereichen nachhaltig negativ beeinflusst: Erstens, die Kinder entbehren die notwendige körperliche Versorgung und Zuwendung; zweitens, die soziale, emotionale und kognitive Entwicklung ist beeinträchtigt; und drittens, die Kinder sind durch den elterlichen Einfluss einem größeren Risiko ausgesetzt, selbst eine Substanzabhängigkeit zu entwickeln (Hogan, 1998). Nach einem kurzen Überblick über die verschiedenen Forschungsansätze beschäftigt sich die vorliegende Arbeit vor allem mit der in Punkt 2 genannten sozialen, emotionalen und kognitiven Entwicklung der Kinder von drogenabhängigen und hier speziell opiatabhängigen Eltern. Dabei geht es, wie im nächsten Kapitel ausgeführt, in der aktuellen Forschung nicht nur um die Frage, ob tatsächlich Beeinträchtigungen vorhanden sind, sondern vielmehr um die Fragen nach den Ursachen einer potentiellen Beeinträchtigung, nach dem Zusammenspiel von biologischen und psychosozialen Einflüssen und der Wirkung von Risiko- und Schutzfaktoren. Auch wenn es in dieser Arbeit speziell um die Entwicklung von Kindern opiatabhängiger Mütter geht, nimmt im theoretischen Teil die Darstellung von Ergebnissen zur Entwicklung der Kinder kokainabhängiger Mütter einen mindestens ebenso großen Teil ein. Dies liegt vor allem daran, dass die internationale Forschung sich, wie oben beschrieben, auf diese Gruppe konzentriert hat. Speziell die diesem Forschungsfeld immanenten methodischen Probleme, die mit der Frage verknüpft sind, wie sich der Beitrag der Umweltbedingungen vom Beitrag der somatischen Vulnerabilität trennen lässt, treffen auf Studien zur kindlichen Entwicklung Opiatabhängiger oder Kokainabhängiger gleichermaßen zu. Andererseits ist die getrennte Betrachtung von Kokain- und Opiatkonsum per se ein theoretisches Hilfskonstrukt. Das oft gleichzeitige Bestehen von sowohl Opiat- als auch Kokainkonsum im realen Leben spiegelt sich auch in vielen, vor allem älteren Studien wider, in denen in den Gruppen drogenexponierter Kinder verschiedene Substanzabhängigkeiten nicht voneinander getrennt werden bzw. gleichzeitig bestehen. 


\section{Forschungsansätze}

Die meisten früheren Forschungsarbeiten zur Langzeitwirkung mütterlichen Drogenkonsums auf die kindliche Entwicklung sind dem sogenannten „main effect model of disease“ bzw. dem „teratology model“ zuzuordnen. In diesem Modell wird versucht einen isolierten spezifischen Effekt der in-utero-Drogenexposition auf die kindliche Entwicklung zu entdecken, ohne dabei potentielle konfundierende Variablen zu berücksichtigen. Wie Moe und Slinning (2002) in ihrem Überblick schreiben, sind viele Studien, in denen Langzeitauswirkungen des mütterlichen Drogenkonsums auf die Kinder gefunden wurden, aus diesem und auch aus anderen Gründen in ihrer Aussagekraft geschmälert. Neben der unzulänglichen Kontrolle konfundierender Variablen kritisieren sie vor allem das Fehlen von adäquaten Kontrollgruppen sowie die Verwendung von Einzelfallstudien. Weiterhin konzentriert sich die Forschung vor allem auf Säuglinge und Kleinkinder und es besteht ein eklatanter Mangel an Studien über die Entwicklung von Kindern zwischen 4 und 11 Jahren. Über die psychosoziale Entwicklung älterer Jugendlicher aus drogenbelasteten Familien ist hingegen durch Studien über Prädiktoren jugendlichen Substanzmissbrauchs (z.B. Charvoz, Bodenmann \& Hermann, 2002) wieder deutlich mehr bekannt. Vor allem die frühen Forschungsarbeiten konzentrieren sich fast ausschließlich auf die medizinischen Auswirkungen eines intrauterinen Drogenkonsums und die psychische und emotionale Entwicklung dieser Kinder wurde in der Forschung lange Zeit vernachlässigt. Untersuchungen zu den Auswirkungen der psychosozialen Risiken, die das Aufwachsen bei einer drogenkonsumierenden Mutter bzw. Eltern mit sich bringen, finden sich erst in jüngerer Zeit und die Ergebnisse sind durchaus widersprüchlich (Englert \& Ziegler, 2001).

Aus diesem Grund wird ein Wechsel vom „teratology“ bzw. „main effect model“ zu einem „transactional“ bzw. „multiple risk model“ gefordert (Alessandri, Bendersky \& Lewis, 1998; Moe \& Slinning, 2002; Howard, Beckwith, Espinosa \& Tyler, 1995; Jacobson \& Jacobson, 2001), welches die beiden Forschungsperspektiven - der biologische Einfluss der pränatalen Drogenexposition auf der einen Seite versus der Auswirkung der mit elterlichen Drogenkonsums verbundenen psychosozialen Risiken auf der anderen Seite - miteinander verbindet. Die pränatale Drogenexposition wird dann als ein Risikofaktor innerhalb der Entwicklung gesehen, welcher durch andere Risiko- und Schutzfaktoren weiter beeinflussbar ist. Bei Kindern drogenabhängiger Eltern besteht nach diesem Modell eine biologische 
Vulnerabilität, die sich z.B. äußert in Schwierigkeiten bei der Emotionsregulation und der Fähigkeit, Aufmerksamkeit zu fokussieren und aufrecht zu erhalten (Kaltenbach, 1996; Mayes, 1996). Diese biologische Vulnerabilität macht die Kinder anfälliger für die negativen Auswirkungen einer wenig optimalen Umwelt und die oft mit elterlichem Drogenkonsum assoziierten vielfältigen Risiken. Umgekehrt geht man davon aus, dass dieser biologische Risikofaktor durch eine fördernde Umwelt und kompetente Erziehung auch kompensiert werden kann (Moe \& Slinning, 2002; Mayes, Cicchetti, Acharyya \& Zhang, 2003). Zusammenhänge zwischen pränataler Drogenexposition und der kindlichen Entwicklung werden nicht mehr im Sinne von kausalen Wirkzusammenhängen alleine interpretiert und direkt der Drogeneinwirkung in-utero zugeschrieben, sondern es werden auch andere Variablen und Wirkmechanismen berücksichtigt. Und auch die Drogeneinwirkung an sich ist komplex. So muss unterschieden werden zwischen linearen Zusammenhängen - eine stärkere Exposition bedingt auch eine stärkere Beeinträchtigung - und Schwelleneffekten, bei denen die Substanz erst ab einer bestimmten Dosis oder einer bestimmten Einnahmedauer teratogen wirkt (Mayes et al., 2003).

Die Liste der zu beachtenden Mediator- und Moderatorvariablen ist lang, wobei vor allem verstärkt auch das kindliche Temperament beachtet wird. Weitere zu berücksichtigende Variablen sind auf Seiten des Kindes z.B. das Geschlecht, medizinische Komplikationen, wie etwa ein niedriges Geburtsgewicht, Betreuungswechsel und Platzierung bei Pflege- und Adoptiveltern. Auf Seiten der Eltern gehören dazu die elterliche Psychopathologie, sozioökonomische Risikofaktoren, wie etwa das Bildungsniveau der Eltern oder Arbeitslosigkeit, zusätzlicher Konsum der Eltern von Alkohol, Nikotin bzw. der Missbrauch mehrerer verschiedener Drogen gleichzeitig sowie Substitution (Jacobson \& Jacobson, 2001; Moe \& Slinning, 2002; Richardson, 1998).

Die Vielzahl an möglicherweise wirksamen Faktoren und der Mangel an Erkenntnissen über deren Zusammenwirken macht klar, warum bisherige Forschungsergebnisse oft $\mathrm{zu}$ widersprüchlichen Ergebnissen kommen und warum es kein einheitliches Profil der „Drogenkinder“"geben kann. 


\section{Die Lebenssituation von Kindern süchtiger Eltern -}

\section{Risikofaktoren}

Kinder drogenabhängiger Eltern sind vielfältigen Risikofaktoren ausgesetzt, wobei manche davon als spezifisch für die Situation dieser Kinder gelten und eine direkte Folge des mütterlichen Drogenkonsums sind, wie z.B. die Entwicklung eines neonatalen Abstinenzsyndroms, andere wiederum sind unspezifisch, treten aber bei Kindern suchtkranker Eltern gehäuft auf, wie z.B. wechselnde Stiefväter oder wechselnde Fremdunterbringungen mit einem Verlust der Hauptbezugsperson. Die Risikofaktoren können in medizinische/biologische und psychosoziale Risikofaktoren unterteilt werden.

\subsection{Medizinische Risikofaktoren}

Aufgrund des oft beeinträchtigten Gesundheitszustandes der drogenabhängigen Mütter sowie der oft nicht oder nicht regelmäßig durchgeführten Vorsorgeuntersuchungen (z.B. Bandstra et al., 2002) ist die Rate an Schwangerschafts- und Geburtskomplikationen sowohl beim Heroinals auch beim Kokainkonsum erhöht.

\subsubsection{Spezifische Effekte der Kokain- bzw. Opiatexposition}

In Bezug auf die Auswirkungen des Kokainkonsums wird in der Literatur ein erhöhtes Risiko für Fehl- und Totgeburten, intrauteriner Wachstumsverzögerung und Frühgeburtlichkeit berichtet. Die Kinder haben oft ein niedriges Geburtsgewicht, eine reduzierte Körpergröße und weisen einen kleineren Kopfumfang (Mikrozephalie) auf. Der Einfluss der Kokainexposition auf das Geburtsgewicht zeigt sich allerdings umso stärker, je höher das Gestationsalter ist (Brown, Bakeman, Coles, Demi und Sexson, 1994) und in einer multizentrischen Studie an 11235 Kindern wurde der Unterschied im Geburtsgewicht zwischen exponierten und nicht exponierten Kindern (Kokain und/oder Opiate) erst ab einer Schwangerschaftsdauer von 33 Wochen signifikant (Bada et al., 1996). Während die Kinder bei Gewicht und Körpergröße durchaus wieder aufholen, bleibt der Kopfumfang im Vergleich zu Gleichaltrigen oft dauerhaft geringer (Nulman et al., 1994; Griffith, Azuma \& Chasnoff, 1994). Kokain besitzt eine teratogene Wirkung und im Sinne von Gewebe-destruierenden 
Effekten kann es auch zu Hirninfarkten und Hirnblutungen bei Neugeborenen als Folge des Kokainkonsums kommen (Schulze, Internet).

Im Gegensatz zu Kokain wird bei Heroin keine teratogene Wirkung angenommen (Ornoy \& Arnon, 1993; Topitz, Ortner, Fischer, 2001), Heroinmissbrauch ist aber ebenso wie der Missbrauch von Kokain mit Frühgeburtlichkeit, einem niedrigeren Geburtsgewicht und einem kleineren Kopfumfang assoziiert. Auch wenn die Inzidenz von Fehlbildungen nicht erhöht ist, so wird doch eine unmittelbare Wirkung der Opiate im fetalen Gewebe angenommen. In Tierversuchen fand sich im fetalen Gehirngewebe eine reduzierte Nukleinsäure- und Proteinsynthese mit herabgesetzter Dichte an kortikalen Neuronen und eine Abnahme neuronaler Prozesse nach Heroingabe (Fanaroff \& Martin, 2002).

Generell besteht bei Drogenkonsum für die Neugeborenen ein größeres Infektionsrisiko von Hepatitis B und C sowie HIV (Finnegan \& Ehrlich, 1990) und die Wahrscheinlichkeit für das Auftreten des plötzlichen Kindstod (SIDS) ist deutlich erhöht (Kandall, Gaines, Habel, Davidson \& Jessop, 1993), wobei die Auftretenswahrscheinlichkeit bei Opiatkonsum größer ist als bei Kokainkonsum (Finnegan \& Kandall, 1997). In einer neueren Studie wiesen $75 \%$ der drogenexponierten Kinder medizinische Komplikationen auf im Vergleich zu 27\% der nicht exponierten Kinder (Finnegan, 2000). Viele dieser medizinischen Probleme, wie z.B. Hypoglykämie oder Septikämie, sind aber sekundärer Natur und durch Faktoren wie Frühgeburtlichkeit und niedriges Geburtsgewicht bedingt. Mehrere Befunde zeigen, dass die beschriebenen medizinischen Faktoren einen Risikofaktor für die weitere Entwicklung darstellen. So finden sich z.B. Zusammenhänge zwischen niedrigem Geburtsgewicht und niedrigen Scores im Bayley Mental Development Index (z.B. Messinger et al., 2004; Howard et al., 1995). Im Tierexperiment an Ratten wurde nachgewiesen, dass pränataler Opiatkonsum zu Veränderungen im Gehirn und im Verhalten führt und, dass diese Veränderungen sich nicht spezifisch auf eine Hirnregion oder einen bestimmten Neurotransmitter beziehen (Vathy, 2002).

\subsubsection{Das Entzugssyndrom}

Bedingt durch den mütterlichen Drogenkonsum tritt bei ca. $70 \%$ aller opiatexponierten Neugeborenen innerhalb der ersten Lebenstage das Neonatale Abstinenzsyndrom (NAS) als Zeichen des kindlichen Entzugs auf (Schneider et al., 1996). Bei methadonsubsituierten Müttern tritt der Entzug meist erst 24-72 Stunden nach der Geburt auf, dafür aber meistens auch heftiger als bei Heroinkonsum, was mit der größeren Halbwertszeit zusammenhängt. 
Methadonsubstituierte Kinder haben aber ein geringeres Risiko zu früh und mit geringem Gewicht geboren zu werden: In der an der Universität Frankfurt erhobenen Stichprobe, auf der auch die vorliegende Untersuchung basiert, wurden 54,3\% der Kinder substituierter Mütter normalgewichtig und termingerecht geboren im Gegensatz zu 25,8\% der Kinder nichtsubstituierter, heroinabhängiger Mütter (Ziegler, Poustka, v. Loewenich, Englert, 2000).

Die Entzugserscheinungen beim Opiatentzug wurden von Finnegan (1985) beschrieben und sind gekennzeichnet durch zentralnervöse Symptome (z.B. Tremor, erhöhter Muskeltonus, schrilles Schreien, Krampfanfälle), metabolische/respiratorische Störungen (z.B. Fieber, Schwitzen) und gastrointestinale Störungen (z.B. Erbrechen, Diarrhoe). Abhängig von der Reifung des kindlichen Stoffwechsels können die Symptome in einem Zeitraum von 6 Tagen bis zu 8 Wochen beobachtet werden. Die Ausprägung des NAS korreliert dabei mit der Menge und der Dauer des mütterlichen Opiatkonsums vor der Geburt. Auch andere Drogen, wie Barbiturate, Amphetamine, Kokain oder Benzodiazepine können zentralnervöse Symptome hervorrufen, allerdings treten die Symptome nicht so klar umschrieben auf, und es existiert auch kein Bewertungsschema wie beim opiatinduzierten NAS (Huestis \& Choo, 2002). Möglicherweise handelt es sich hierbei auch weniger um Symptome einer Entzugssymptomatik sondern eher um direkte toxische Wirkungen von z.B. Kokain (Schulze, Internet).

Laut Berger et al. (2002) stellt die Dauer des neonatalen Entzugssyndroms einen zentralen Risikoparameter für die spätere Entwicklung dar.

\subsection{Psychosoziale Risikofaktoren}

Das Risiko der Arbeitslosigkeit ist bei den drogenabhängigen Müttern erhöht (Bandstra et al., 2002) und manche Autoren sprechen von Quoten bis zu 80\% (Perez-Bescos et al., 1993). Aufgrund der dadurch bedingten finanziellen Knappheit leben viele Drogenabhängige von Sozialhilfe, vor allem drogenabhängige Frauen mit Kindern. $\mathrm{Zu}$ den spezifischen Risikofaktoren drogenabhängiger Frauen gehören auch Inhaftierungen, Obdachlosigkeit und Prostitution. Die Partnerbeziehung gestaltet sich meistens instabil, es gibt häufige Trennungserfahrungen und viele Mütter sind alleinerziehend (Klein, 2001). Die verbreitete soziale Isolierung führt $\mathrm{zu}$ emotionalen Problemen und auch dazu, dass Mütter in Stresssituationen oft keine soziale Unterstützung erfahren. Häufig finden sich bei drogenabhängigen Müttern auch psychiatrische Auffälligkeiten. Dazu gehören vor allem depressive Verstimmungen, aber auch antisoziale Persönlichkeitszüge (Nair et al., 1997; 
Kandel, 1984; Kandel, Murphy \& Karus, 1985). Nair et al. (2003) fanden in ihrer Untersuchung zu Risikofaktoren substanzabhängiger Mütter, dass im Zeitraum von 18 Monaten nach der Geburt des Kindes 70\% der Mütter über depressive Symptome klagten, 20 $\%$ waren inhaftiert und $13 \%$ waren obdachlos. Nur $13 \%$ lebten in einer kontinuierlichen Beziehung.

Diese schwierigen psychosozialen Bedingungen, fehlende Unterstützung und eigene psychische Probleme führen auch zu einer Überforderung in Bezug auf die Kindererziehung. Oft leidet darunter die Qualität des mütterlichen Erziehungsverhaltens und in Folge auch die Mutter-Kind-Beziehung. Speziell mit diesen Aspekten elterlichen Erziehungsverhaltens beschäftigt sich das nächste Kapitel. 


\section{Drogenabhängige und Elternschaft}

Innerhalb der Gesellschaft und auch in der Forschung wird implizit davon ausgegangen, dass substanzabhängige Eltern ihre Kinder nur unzulänglich versorgen. Diese Annahme ist sicher nicht unberechtigt, die Anzahl der Studien, die auf die Qualität der Erziehung und Versorgung von Kindern drogenabhängiger Eltern fokussieren, ist aber nur begrenzt. Die untersuchten Indikatoren reichen dabei von eindeutigem elterlichen Fehlverhalten, wie etwa körperlicher Misshandlung und Vernachlässigung, hin zu weniger eindeutigen und subtileren Faktoren, wie sie in bestimmten Aspekten der Eltern-Kind-Interaktion zu Tage treten (Hans, 2002). Deutlich besser untersucht ist das Verhalten alkoholabhängiger Eltern. Einige der Charakteristika des Erlebens von Kindern alkoholabhängiger Eltern (z.B. Klein 2002) lassen sich wahrscheinlich auf die Situation von Kindern drogenabhängiger Eltern übertragen. Dazu gehört: Das Erleben dieser Kinder ist gekennzeichnet durch größere Disharmonie zwischen den Eltern und größere Stimmungsschwankungen und Unberechenbarkeiten im Verhalten gegenüber den Kindern. Sie sind häufiger Loyalitätskonflikten zwischen den Eltern ausgesetzt, es fehlen ein strukturierter Alltag sowie Verlässlichkeiten im familiären Ablauf. Das Risiko für Missbrauch und Vernachlässigung ist erhöht. Vaillant und Milofsky (1982) führen als Gemeinsamkeit im Erziehungsverhalten drogensüchtiger und alkoholkranker Eltern an, dass oft extreme Disziplinierungsstile vorherrschen (Außergewöhnliche Strenge oder sehr nachlässiges Verhalten) sowie oft unrealistisch hohe Erwartungen an die Kinder, wie z.B. mit Hilfe des Kindes den Ausstieg aus der Drogenszene zu schaffen. Wie Kandel in einer Studie (1990) beschreibt, sinkt die Fähigkeit zu adäquatem Elternverhalten mit dem Ausmaß der elterlichen Drogenproblematik. Die Stärke der Drogenproblematik und das Vorhandensein von schwerem Alkoholmissbrauch waren assoziiert mit einem sehr strafenden Erziehungsstil, der durch einen Mangel an Wärme, Nähe und positiver Interaktion mit dem Kind gekennzeichnet war, ebenso wie größere Unstimmigkeiten mit dem Partner über die Erziehung des Kindes.

Zu Recht wird aber darauf hingewiesen (Beeghly, Frank, Rose-Jacobs, Cabral und Tronick, 2003; Velez et al., 2004), dass vom elterlichen Drogenkonsum nicht per se auf ein wenig optimales Erziehungsverhalten oder eine schlechte Mutter-Kind-Interaktion geschlossen werden darf. Vielmehr wird das Erziehungsverhalten drogenabhängiger Eltern durch die oben beschriebenen Faktoren wie Armut, chaotische Lebensumstände, geringe Schulbildung der 
Eltern, psychische Probleme der Eltern, oder auch durch die in-utero-Exposition bedingte Irritabilität des Kindes an sich negativ beeinflusst und das Auftreten eines wenig adäquaten Erziehungsverhaltens wird begünstigt. Viele drogenabhängige Frauen haben in ihrer Kindheit selbst Traumen wie Missbrauch und Vernachlässigung erfahren und sind aus diesem Grund in ihrem Erziehungsverhalten ineffektiv und ambivalent (Davis, 1990). Je höher die zusätzlichen medizinischen und psychosozialen Risiken, desto größer ist auch die Wahrscheinlichkeit, dass Eltern ihre Kinder nicht ausreichend gut versorgen können, und desto größer ist auch der subjektiv wahrgenommene Stress der Eltern (Nair et al., 2003).

\subsection{Einstellungen und Werthaltungen der Eltern}

Drogenabhängige Mütter sehen sich dem Dilemma gegenüber, einerseits ihre Kinder optimal versorgen $\mathrm{zu}$ wollen, dies andererseits aber unter meist schwierigen psychosozialen Bedingungen tun zu müssen. Alleine der Drogenbeschaffungsdruck führt notwendigerweise dazu, dass für Erziehungstätigkeit und positive Zuwendung weniger Zeit bleibt. Drogenabhängige Mütter sind deshalb häufiger als andere Mütter der Meinung, als Eltern ungeeignet $\mathrm{zu}$ sein und sorgen sich mehr um die Zukunft ihrer Kinder, insbesondere hinsichtlich eines Abrutschens in Drogenabhängigkeit und Dissozialität (Colten, 1980). Die zukünftige Drogenabhängigkeit ihrer Kinder sehen diese Mütter aufgrund der in-uteroExposition oft als unabänderliche Tatsache (Velez et al., 2004). Fehlinformationen wie diese tragen zu Schuldgefühlen und Hoffnungslosigkeit bei den Müttern und zum „labeling“ der Kinder bei, dabei wollen die Mütter wie die meisten „,normalen“ Eltern auch ihre Kinder optimal und in einem adäquaten Umfeld versorgen. Richter und Bammer (2000) zeigten in ihrer qualitativen Studie an 22 heroinabhängigen Müttern, mit welchen Strategien diese versuchen, die durch ihren eigenen Substanzkonsum entstandenen negativen Effekte und Risiken ihres Lebensstils auf ihre Kinder zu reduzieren. Sieben unterschiedliche Strategien, im Folgenden von am „meisten erstrebenswert“ bis am „wenigsten erstrebenswert“ hierarchisch angeordnet, wurden identifiziert: 1. Vollständige Beendigung des Substanzkonsums; 2. Behandlung, vor allem Methadonsubstitution; 3. das Ausmaß der konsumierten Substanz bleibt klein und stabil; 4. Abschirmen der Kinder von mit Drogen in Verbindung stehenden Aktivitäten; 5. Bemühung, die häusliche Umgebung stabil und sicher $\mathrm{zu}$ halten; 6. Vermeidung von Inhaftierung und 7. Platzierung der Kinder bei einer verantwortungsbewussten Betreuungsperson bei gleichzeitiger bestmöglicher 
Aufrecherhaltung der eigenen elterlichen Rolle. Besonders die letztgenannte Strategie versteht sich als letzter Ausweg, wenn die körperlichen und psychischen Auswirkungen der Sucht eine angemessene Ausübung der Elternrolle unmöglich machen.

\subsection{Qualität der Eltern-Kind-Interaktion}

Trotz der in 4.1 beschriebenen Wünsche und Bestrebungen der Eltern, eine möglichst optimale Erziehung zu gewährleisten, weist eine Reihe von Studien darauf hin, dass die Eltern-Kind-Interaktion zwischen drogenabhängigen Müttern und ihren Kindern problematisch und durch unzureichendes und inadäquates Erziehungsverhalten gekennzeichnet ist (Fitzgerald, Kaltenbach \& Finnegan, 1990; Bernstein, Jeremy \& Marcus, 1986).

Als Ursachen dafür sind einerseits Regulationsschwierigkeiten und eine erhöhte Irritabilität des Kindes zu nennen. Bei Kindern mit pränataler Kokainexposition wurde in mehreren Studien in Bezug auf stressreiche Situationen entweder eine geringe emotionale Ansprechbarkeit und affektive Expressivität des Kindes (z.B. Alessandri, Sullivan, Imaizumi \& Lewis, 1993, Mayes et al., 1997; Mayes et al, 1998; Ukeye et al., 2001) oder auch eine Neigung zu erhöhter emotionaler Dysregulation (Bendersky \& Lewis, 1998; Mayes et al.,1996) festgestellt; beides Reaktionsstile, die eine Herausforderung an das Erziehungsverhalten darstellen und die Wahrscheinlichkeit für Schwierigkeiten in der ElternKind-Beziehung erhöhen. Andererseits darf aber auch hier nicht von simplen UrsacheWirkungs-Zusammenhängen ausgegangen werden, sondern neben den biologischen Risiken können die psychosozialen Gegebenheiten aggravierend oder kompensierend wirken, wie folgende Untersuchungen verdeutlichen: Jeremy und Bernstein (1984) zeigten, dass opiatabhängige Mütter (in diesem Fall methadonsubsitutiert) zwar eine weniger gute MutterKind-Interaktion aufwiesen als die Vergleichsgruppe, allerdings erwies sich der Einfluss der Methadonsubstitution nicht als direkter Wirkfaktor, sondern die Qualität der Eltern-KindInteraktion stand in starkem Zusammenhang mit Faktoren wie der mütterlichen Intelligenz, der Schulbildung, der Schwere der Psychopathologie und dem Beziehungsstatus zum Vater des Kindes. Das Ausmaß der pränatalen Kokainexposition zeigte in der Untersuchung von Beeghly et al. (2003) keinen direkten Zusammenhang mit einer höheren Rate von unsicherem Bindungsverhalten einjähriger Kinder in der fremden Situation nach Ainsworth. Bei hohem pränatalen Kokainkonsum in Kombination mit geringer Unterstützung öffentlicher Einrichtungen zeigten sich aber vermehrt subtilere Veränderungen im Bindungsverhalten der 
Kinder (weniger Weinen, größeres Vermeidungsverhalten), ohne dass dies insgesamt zu einer Zuordnung zur Kategorie unsicherer Bindung führte.

Einen weiteren Aspekt führen Velez et al. (2004) auf, wonach ein Wissensmangel und falsche Annahmen über die adäquate Erziehung der Kinder großen Einfluss auf die Eltern-KindInteraktion hat. So zeigte sich, dass in einer Gruppe schwangerer, vor allem opiatabhängiger Frauen die Einstellung vorherrschte, dass ein weinender Säugling durch Hochnehmen und Halten verwöhnt werde und es daher besser sei, die Babys schreien zu lassen. Die Aufklärung darüber, dass das Hochnehmen, besonders in den ersten Monaten, eine richtige Reaktion ist, wirkt sich auf die Mutter-Kind-Beziehung förderlich aus und kann dabei helfen, den Teufelskreis einer negativen Mutter-Kind-Interaktion und einer daraus oft resultierenden unsicheren Bindung zu unterbrechen. Eine unsichere Bindung gilt als Risikofaktor für spätere emotionale Verhaltensauffälligkeiten (Belsky \& Pasco Fearon, 2002; Kochanska, 2001; Urban et al., 1992), im Sinne des Teufelkreises kann es in der Folge zu einer Überforderung der Mutter, zu Schuldgefühlen über das Versagen in der Erziehung, bis zu Vernachlässigung und Misshandlung des Kindes kommen.

\subsection{Kindesmisshandlung und -vernachlässigung}

Das gemeinsame Auftreten von Substanzabhängigkeit und einem erhöhtem Risiko für Kindesmisshandlung ist ein durchgängiges Ergebnis verschiedener Untersuchungen. In ihrer retrospektiven Studie zum Zusammenhang von Drogenmissbrauch und Kindesmisshandlung konnten Kienberger Jaudes et al. (1995) zeigen, dass die Kinder von während der Schwangerschaft drogenmissbrauchenden Müttern (Opiate, Kokain, Marihuana) einem 2-3 mal höheren Risiko für Missbrauch und Vernachlässigung ausgesetzt waren als Kinder aus der allgemeinen Bevölkerung aus der selben Nachbarschaft. Bei allen Kindern, die in einem Zeitraum von fünf Jahren in einem städtischen Krankenhaus geboren worden waren und bei denen eine Drogenexposition in-utero festgestellt worden war, wurde einige Jahre später überprüft, ob es zu einer Anzeige wegen Kindesmisshandlung gekommen war. Bei 155 der 513 Kinder war dies der Fall, bei 102 davon wurden die Vorwürfe auch bestätigt. Die mit Abstand häufigste Form der Kindesmisshandlung (72,6\%) stellte dabei die Vernachlässigung dar, gefolgt von körperlichem Missbrauch (15,7\%), anhaltender Drogenexposition (5,9\%) sowie sexuellem Missbrauch (2,9\%) und Tod durch Vernachlässigung (2,9\%). In 88,2\% der Fälle waren die Eltern für die Misshandlung verantwortlich. Variablen wie Alter der Mutter, 
Beziehungsstatus oder Anzahl der Kinder insgesamt hatten keinen Einfluss; das Misshandlungsrisiko war aber bei Müttern mit höherem Schulabschluss (high school oder College) signifikant erhöht. Eine mögliche Erklärung für diesen ungewöhnlichen Befund könnte nach Ansicht der Autoren sein, dass bei substanzabhängigen Müttern mit guter Schulbildung auch die Frustration über das Nichterreichen des eigenen Potentials größer ist.

Nair et al. (2003) ermittelten bei drogenabhängigen Frauen (Kokain oder Heroin) mit einem hohen Risikostatus ein höheres Potential für Missbrauch und Vernachlässigung als bei Müttern mit geringem Risikostatus. Das Risikoniveau wurde kumuliert aus folgenden Faktoren: Mütterliche Depression, familiäre und außerfamiliäre Gewalt, Familiengröße, Inhaftierungen, soziale Isolation, negative Ereignisse, psychiatrische Probleme, Obdachlosigkeit, Schwere des Drogenmissbrauchs.

In einer Studie an 639 Kindern, die aufgrund von Misshandlung aus ihrer Familie herausgenommen wurden, ließ sich bei $79 \%$ der betreuenden Personen Drogenmissbrauch nachweisen (Besinger, Garland, Litrownik \& Landsverk, 1999). Murphy et al. (1991) fanden in ihrer Untersuchung an 206 missbrauchten oder vernachlässigten Kindern bei 43\% davon bei mindestens einem Elternteil Drogen- oder Alkoholmissbrauch. In einer großen repräsentativen Stichprobe untersuchten Walsh, MacMillan \& Jamieson (2003) die Assoziation zwischen elterlichem Alkohol- oder Drogenmissbrauch und körperlichem oder sexuellem Missbrauch an den Kindern. Basierend auf den retrospektiven Angaben der befragten Personen (ab einem Alter von 15 Jahren) ermittelten die Autoren ein mehr als doppelt so hohes Risiko für körperlichen oder sexuellen Missbrauch bei Vorhandensein elterlichen Alkohol- oder Drogenmissbrauchs. Das geringste Risiko lag bei väterlichem Alkohol- /Drogenkonsum vor, das höchste bei Alkohol-/Drogenkonsum beider Elternteile.

Egami et al. (1996) hingegen fanden, dass Substanzmissbrauch mit Kindesvernachlässigung, nicht aber mit Kindesmissbrauch assoziiert war. Soziodemographische Einflüsse und psychiatrische Variablen waren dabei kontrolliert. Auch Chaffin, Kelleher \& Hollenbert (1996) fanden in ihrer Studie, dass substanzmissbrauchende Eltern ein 2,9- bzw. 3,24fach erhöhtes Risiko für Missbrauch und Vernachlässigung gegenüber Eltern ohne Substanzabhängigkeit aufwiesen. Der Faktor Substanzmissbrauch (Drogen- oder Alkohol) erwies sich in dieser Studie als weitaus stärkerer Prädiktor als soziale und demographische Variablen wie Alter, Geschlecht, Schulbildung oder sozioökonomischer Status. 
Die letztgenannte Studie dürfte ein eher untypisches Bild zeichnen, denn bei elterlichem Substanzmissbrauch besteht zwar ein erhöhtes Risiko für Kindesvernachlässigung und Kindesmisshandlung; dieses erhöhte Risiko wird aber weniger als direkte Ursache der Drogenabhängigkeit interpretiert, sondern eher als ein durch Armut und generell schwierige psychosoziale Umstände bedingtes Risiko.

\subsection{Fremdplatzierungen - Unterbrechung der Beziehung zu den}

\section{Eltern}

Für Kinder drogenabhängiger Eltern besteht ein erhöhtes Risiko einer Fremdplatzierung. Ihre Eltern sind wegen ihrer Abhängigkeit von Krankheit und frühem Tod bedroht, werden öfters inhaftiert und müssen aufgrund ihrer Überforderung im Erziehungsverhalten und psychosozialen Mängeln die Erziehung ihrer Kinder abgeben bzw. geben sie freiwillig ab. Entschließen sich drogenabhängige Mütter zu einer Behandlung, sehen sie sich oft einem Dilemma gegenüber. Während sie auf der einen Seite darauf hoffen, dass der Beginn einer Behandlung auch dazu beitragen kann, die eigenen Kinder behalten zu können, fürchten diese Frauen auf der anderen Seite, dass durch einen solchen Schritt erst die Gefahr entsteht, das Sorgerecht für die eigenen Kinder zu verlieren (Powis, Gossop, Bury, Payne \& Griffiths, 2000)

Einige Zahlen verdeutlichen die hohe Rate der Fremdunterbringung innerhalb der ersten Lebensjahre. So fanden Nair et al. (2003), dass im Alter von 18 Monaten 16\% einer Gruppe von Kindern drogensüchtiger Mütter fremdplatziert waren. Bandstra et al. (2002) berichten, dass im Alter von 3 Jahren 73\%, im Alter von 5 Jahren 67,6 \% und im Alter von 7 Jahren nur noch 58,9\% von der leiblichen Mutter betreut wurden. In einer deutschen Untersuchung (Bunikowski et al., 1998) verringerte sich die Rate der bei der leiblichen (opiatabhängigen) Mutter lebenden Kinder von 64,7\% zum Zeitpunkt der Entlassung aus dem Krankenhaus auf 35,3\% im Alter von einem Jahr. Berger und Elstner (2002) fanden für den Raum Wien eine Fremdbetreuungsrate von $32,6 \%$ unmittelbar nach der Geburt; am Ende des Beobachtungszeitraums von 6 Jahren war diese Rate auf 48,8\% gestiegen.

Viele Kinder drogenabhängiger Mütter werden von den Großeltern, und hier besonders von den Großmüttern versorgt. Dies hat den Vorteil, dass die Kinder nicht in eine fremde Umgebung zu ihnen unbekannten Leuten kommen, wie es bei professionellen Pflegefamilien 
der Fall ist. Auf der anderen Seite sind die Großmütter im Normalfall deutlich älter, haben mit größeren gesundheitlichen Problemen zu kämpfen und verfügen oft nur über geringe finanzielle und soziale Ressourcen (Berrick, Barth \& Needell, 1994; Dubowitz et al., 1994, Richter \& Bammer, 2000). Erfolgt die Vermittlung in Pflegefamilien, dann geschieht dies bei Kindern drogenabhängiger Mütter oft früher und für einen längeren Zeitraum als bei anderen Kindern. Speziell mit der Situation adoptierter Kinder mit pränataler Drogenexposition beschäftigt sich Johnson (2002) in ihrem Übersichtsartikel zur kindlichen Entwicklung adoptierter Kinder.

\subsubsection{Risiken für Fremdunterbringung}

Untersuchungen zu Risikofaktoren, welche die Wahrscheinlichkeit eines Sorgerechtverlustes bzw. einer Fremdunterbringung erhöht, erbringen unterschiedliche Ergebnisse: So fanden Tyler, Howard, Espinosa und Doakes (1997), dass Mütter, die das Sorgerecht für ihre Kinder verloren hatten, sich hinsichtlich Alter, Schulbildung, Anzahl der Kinder, Ausmaß des Drogenkonsums und psychischer Gesundheit nicht von den Müttern unterschieden, die ihre Kinder weiter betreuten. Ebenso unterschieden sich die fremduntergebrachten Säuglinge, z.B. hinsichtlich Gestationsalter und Geburtsgewicht, nicht von den bei der Mutter verbliebenen Säuglingen.

Zu ganz anderen Ergebnissen kamen Nair et al. (1997) und sie konnten spezielle Konstellationen von Risikofaktoren für die Diskontinuität in der Beziehung zu den Bezugspersonen eruieren: Die Wahrscheinlichkeit für eine Fremdunterbringung innerhalb der ersten 18 Monate war größer, wenn die Mütter ein jüngeres Lebensalter aufwiesen, wenn sie bereits andere Kinder in Pflegeverhältnissen hatten, während der Schwangerschaft Heroin konsumiert hatten und über mehr depressive Symptome berichteten. Ziegler (1998) zeigte in seiner Untersuchung, die auch die Basis dieser Arbeit bildet, dass die Mütter von schon zum Zeitpunkt der Geburt fremdplatzierten Kindern zu einem überwiegenden und statistisch signifikant größeren Anteil als die Gruppe der Müttern nicht-fremdplatzierter Kinder in instabilen Verhältnissen lebte, d.h. die Mutter lebte allein oder mit wechselnden Partnern, verfügte über keinen festen Wohnsitz und finanzierte ihren Lebensunterhalt überwiegend durch Schattenwirtschaft bzw. Prostitution. Fast die Hälfte der Mütter fremdplatzierter Kinder gehörte der unteren Unterschicht an. Das Fürsorgeverhalten der Mütter war in $75 \%$ der Fälle mangelhaft bzw. kümmerte sich die Mutter überhaupt nicht um ihr Kind, während das 
Fürsorgeverhalten der Mütter nicht-fremdplatzierter Kinder in fast drei Viertel aller Fälle gut bis sehr gut war.

\subsubsection{Substitution und Fremdplatzierung}

Wenig bis gar nicht erforscht ist in diesem Zusammenhang auch die Auswirkung der Substitution. Die Substitution ist durch die kontrollierte und regelmäßige Abgabe und den Wegfall der Beschaffungskriminalität oft der erste Schritt in Richtung Normalität. Die psychosozialen Verhältnisse sind nicht zuletzt dadurch besser und durch die Substitution werden immer weniger Kinder fremdplatziert. Ziegler (1998) ermittelte, dass bei den substituierten Müttern 76,5\% der Kinder nach der Geburt zu den Müttern nach Hause entlassen werden, während es bei den nicht-substituierten Müttern nur 54,2\% waren. Dies ist einerseits natürlich als Erfolg zu werten, andererseits fehlen Untersuchungen über langfristige Weiterentwicklungen. Die Inanspruchnahme von Vorsorgeuntersuchungen und die Akzeptanz für soziale Unterstützung ist bei methadonsubstituierten Frauen zwar größer (Soeptami, 1994), die Methadonsubstitution darf aber nicht als Allheilmittel missverstanden werden und es bleibt offen, ob die dadurch erreichte psychosoziale Stabilisierung die fortbestehenden Risiken, etwa bedingt durch Beikonsum anderer Drogen (Arnold, Feldmeier-Thon, Fritsch \& Simmedinger, 1995), kompensieren können. Es ist möglich, dass Kinder substituierter Eltern zwar zunächst eher bei ihren Eltern verbleiben, dass es aber zu einem späteren Zeitpunkt doch noch zu einer Fremdplatzierung kommt und durch den späten Bezugspersonenwechsel das Risiko für Verhaltensauffälligkeiten, z.B. durch die längere Exposition gegenüber malignen elterlichen Verhaltensweisen, bei den Kindern im Endeffekt größer ist, als wenn der Betreuungswechsel früh stattgefunden hätte (Soeptami, 1994; Fanshel, 1975). 


\section{Entwicklung der Kinder substanzabhängiger Eltern}

Resultate zur Entwicklung der Kinder sind wenig einheitlich und während manche Studien geringe bis mittelgradige Entwicklungsrückstände bei Kindern drogenabhängiger Eltern im Vergleich zu Kindern einer Kontrollgruppe fanden, sind laut der Ergebnisse anderer Untersuchungen, keine Unterschiede festzustellen. Besonders wenn es um die kognitive Entwicklung geht, sind vor allem jüngere Kinder Gegenstand der Forschung, während die Aussagen über Kinder im Schulalter nur spärlich vorhanden sind. Das sehr unterschiedliche Lebensalter der Kinder, in dem mögliche Effekte der Drogenexposition untersucht werden, ist möglicherweise ein Teil der Ursache für die oft gegensätzlichen Ergebnisse in Bezug auf die Existenz von Entwicklungsdefiziten, denn es gibt Hinweise darauf, dass Defizite sich umso stärker manifestieren, je älter die Kinder sind und je mehr sich komplexere intellektuelle Fähigkeiten entwickeln (Griffith, Azuma \& Chasnoff, 1994; Berger \& Elstner, 2002). Auch der Grad der Drogenexposition spielt eine Rolle. Niedrige Drogen-Expositionsniveaus verursachen keine morphologischen Veränderungen, führen aber wahrscheinlich zu Effekten im zentralen Nervensystem, die sich erst mit zunehmendem Alter der Kinder niederschlagen (Richardson, 1998, Mayes, 2003). Im Falle einer weiterbestehenden Drogenabhängigkeit der Eltern spielt auch die Drogenexposition durch passive Inhalation und dadurch bedingte Beeinträchtigungen in der neuronalen Entwicklung eine Rolle (Mayes et al., 2003). Auf der anderen Seite sind Entwicklungsdefizite bei älteren Kindern auch bedingt durch schon länger wirkende psychosoziale Risikofaktoren (Hans \& Jeremy, 2001) und ein größerer Leistungsabfall ist mit Sicherheit kein spezifischer Effekt der Drogenexposition sondern ein allgemeines Phänomen einer Hoch-Risiko-Population - unter anderem begründet durch schlechte Förderung, geringe Stimulation und allgemein wirkende Stressfaktoren. Letztendlich ist mit Sicherheit die in der bisherigen Forschung sehr unterschiedlich gehandhabte Berücksichtigung von konfundierenden Variablen für die teilweise widersprüchlichen Ergebnisse mitverantwortlich.

\subsection{Kognitive Entwicklung}

Der Großteil aktueller Studien beschränkt sich auf die Entwicklung von Kindern von kokainsüchtigen Müttern. Wie praktikabel dieses Vorgehen bzw. die Konzentration auf eine spezifische Droge ist, und damit auch die Repräsentativität der Ergebnisse, wird in der 
Literatur durchaus kritisch diskutiert (z.B. Moe \& Slinning, 2002), vor allem da fast immer von einem Beikonsum anderer illegaler Drogen sowie von Alkohol- und Nikotinkonsum ausgegangen werden kann.

\subsubsection{Effekte von Kokain}

Schon in frühen Studien, in denen konfundierende Variablen nur wenig Berücksichtigung fanden und in denen oft nicht unterschieden wurde, ob es sich um eine pränatale oder eine postnatale Drogenexposition bzw. um beides handelte, zeichnete sich schon die dieses Forschungsfeld prägende Widersprüchlichkeit der Ergebnisse ab. So fand Fanshel (1975) im Vergleich von Kindern drogenkonsumierender Eltern bei Pflegefamilien mit anderen Pflegekindern keine Hinweise auf Defizite in der kognitiven Entwicklung, während Nichtern (1973) bei Kindern drogenabhängiger Eltern in Pflege durchaus Verzögerungen in der Sprachentwicklung sowie Lernprobleme in der Schule feststellte.

In Studien jüngeren Datums zeigt sich durchgehend der Versuch, genauere Aussagen über die Ursache-Wirkzusammenhänge möglicher Defizite treffen zu können und einige Studien haben sich speziell mit den unterschiedlichen Effekten unterschiedlicher Dosierungen bzw. unterschiedlicher Drogen beschäftigt: Alessandri et al. (1998) variierten das Ausmaß der pränatalen Drogenexposition und verglichen Kinder mit einer hohen in-utero-KokainExposition mit Kindern die dieser Substanz pränatal nur schwach ausgesetzt waren im Alter von 8 und 18 Monaten. Zum ersten Untersuchungszeitpunkt zeigten sich keine Unterschiede zu einer Kontrollgruppe, zum Zeitpunkt der zweiten Untersuchung war die kognitive Leistungsfähigkeit der stark exponierten Kinder gesunken (gemessen mit dem Bayley Mental Developmental Index, MDI) und im Vergleich zur Kontrollgruppe signifikant niedriger. Keine Unterschiede bezüglich der intellektuellen Entwicklung konnten hingegen Hurt et al. (1997) finden. Der Vergleich von Kindern mit starker und schwacher in-utero-KokainExposition erbrachte keine Unterschiede im Mental Developmental Index und auch die Gesamtgruppe der drogenexponierten Kinder erzielte im Vergleich zur Kontrollgruppe vergleichbare Ergebnisse im Wechsler Preschool and Primary Scale of Intelligence-Revised. Bei dieser Studie bemerken die Autoren selbst allerdings kritisch, dass die in allen Gruppen durchgängig schwachen und gegenüber dem erwarteten Mittelwert reduzierten Ergebnisse der Intelligenztests durch den sehr schwachen sozioökonomischen Status der getesteten Kinder 
begründet und dieser Effekt so stark sein könnte, dass auf den teratogenen Einfluss der Drogen zurückzuführende Auswirkungen dadurch verwischt worden sein könnten.

Den Effekten unterschiedlicher Drogen haben sich z.B. Griffith et al. (1994) gewidmet. Sie untersuchten Kinder kokainabhängiger Mütter deren Beikonsum keine Opiate enthielt (Gruppe 1), Kinder substanzmissbrauchender Mütter (eine Kombination von Marihuana, Alkohol und Opiaten) die kein Kokain konsumierten (Gruppe 2) sowie eine Kontrollgruppe ohne Drogenkonsum (Gruppe 3). Im Stanford-Binet Intelligenztest zeigten sich in der Gesamtintelligenz keine Unterschiede zur Kontrollgruppe, die Kinder der Gruppe 1 erzielten aber signifikant niedrigere Werte beim verbalen Schlussfolgern (Verbal Reasoning), während die Gruppe 2 vor allem in Bezug auf abstraktes/visuelles Schlussfolgern (Abstract/Visual Reasoning) schlechter abschnitt als die Kontrollgruppe. Die drogenkonsumierenden Mütter in dieser Studie wiesen zwar einen niedrigen sozioökonomischen Status auf, waren aber schon in der 15. Schwangerschaftswoche rekrutiert worden, und erhielten intensive gynäkologische und psychotherapeutische Unterstützung. Sie stellten damit innerhalb der Drogenpopulation eine Sondergruppe dar, was die Ergebnisse nur schlecht verallgemeinerbar macht. Das unterschiedliche Abschneiden von Gruppe 1 und 2 kann aber im Sinne drogenspezifischer Effekte interpretiert werden. Mayes et al. (2003) verglichen drei Gruppen miteinander, die alle einen sehr niedrigen sozioökonomischen Status aufwiesen und aus ärmlichen und sozial instabilen Verhältnissen kamen. Gemessen mit dem Bayley Mental Development Index zeigte die Gruppe der in-utero-Kokain-exponierten Kinder eine niedrigere Leistung als eine Kontrollgruppe und eine Gruppe von Kindern, die anderen Drogen wie Alkohol, Tabak oder Marihuana ausgesetzt waren. Ähnlich wie Alessandri et al. (1998) zeigte sich bei der Kokainexponierten Gruppe auch über verschiedene Messzeitpunkte im Alter zwischen 3 und 36 Monaten ein größerer Abfall der Leistung als bei den beiden anderen Gruppen, die allerdings ebenfalls beide eine Leistungsverminderung zeigten. Die Autoren erklären diesen Leistungsabfall nicht mit einem spezifischen Drogeneffekt sondern als einen eher allgemeinen Effekt einer Hoch-Risiko-Gruppe, bedingt durch die psychosozialen Risiken. Dementsprechend zeigte sich auch, dass Geburtsgewicht und Gestationsalter als Mediatorvariablen wirken und das Abschneiden im Mental Development Index signifikant vorhersagen können.

Die Effekte des Zeitpunkts der Dogenexposition war Gegenstand der Untersuchung von Richardson (1998). Die Kinder von drogenkonsumierenden Müttern, die an einer Klinik in der Schwangerschaftsvorsorge eingebunden waren und bereits im ersten Trimester täglich 
Kokain konsumiert hatten, hatten im Alter von drei Jahren ein signifikant niedrigeres Ergebnis im Stanford-Binet Intelligenztest als die Kinder deren Mütter im ersten Trimester keinen oder nur einen geringen Kokainkonsum aufwiesen.

In einer Reihe von weiteren Studien wurden Entwicklungsdefizite der Kinder kokainabhängiger Mütter ermittelt und Nulman et al. (1994) nehmen für sich in Anspruch, die erste Studie durchgeführt zu haben, welche einen neurotoxischen Effekt einer pränatalen Kokainexposition dokumentiert. Kinder mit pränataler Kokainexposition, die bei Adoptivmüttern aufwuchsen, schnitten im Vergleich zu Kindern einer Kontrollgruppe in einem Sprachtest (Reynell Language Test) sowohl beim verbalen Verständnis als auch bei der expressiven Sprache signifikant schlechter ab. Dem Sprachtest wird von den Autoren eine hohe Sensitivität für neurotoxikologische Schädigungen zugesprochen. Adoptivmütter und Kontrollmütter waren in Bezug auf Intelligenz und sozioökonomischen Status parallelisiert und die Adoption erfolgte kurz nach der Geburt, so dass davon ausgegangen werden kann, dass der Einfluss der Umweltbedingungen soweit wie möglich konstant gehalten werden konnte. Gestationsalter, Geburtsgewicht und Parität waren ebenfalls kontrolliert. Bandstra et al. (2002) ziehen in ihrer Studie ebenfalls den Schluss eines direkten kokain-spezifischen Effekts auf die auch in diesem Fall untersuchte sprachliche Entwicklung. Es bestand eine Assoziation zwischen dem pränatalen Kokainkonsum und dem Auftreten von sprachlichen Defiziten, auch wenn potentiell konfundierende Variablen wie Geschlecht des Kindes, pränatale Exposition durch andere Drogen, Geburtsgewicht, Gestationsalter und soziodemographische Variablen kontrolliert wurden. Die Studie von Arendt et al. (2004) gehört zu den wenigen Untersuchungen von Schulkindern und zeigt, wie wichtig es ist, auch die Umweltfaktoren zu berücksichtigen, besonders da mit zunehmendem Alter der Einfluss dieser Umweltbedingungen auf die Entwicklung der Kinder immer größer wird. Im Vergleich zu einer Kontrollgruppe erzielten die Kinder mit pränataler Drogenexposition zwar signifikant niedrigere Ergebnisse in Gesamt-IQ und Verbal-IQ der WASI (Wechsler Abbreviated Scale of Intelligence) und dem VMI (Test of Visual Motor Integration), dieser Effekt verschwand aber als der passive Wortschatz der Mutter (gemessen mit dem Peabody Picture Vocabulary Test, PPVT) sowie ein Maß für die Qualität der häuslichen Umgebung und Entwicklungsbedingungen (Middle Childhood Home Observation for Measurement of the Environment, HOME) als Risikofaktoren in die Analyse inkludiert wurden.

Auch Phelps, Wallace \& Bontrager (1997) konnten keinen direkten Einfluss der in-uteroDrogenexposition finden. Beim Vergleich einer Gruppe 3-6jähriger Kinder mit einer 
Kokain/Polydrogen-Exposition in-utero und einer Kontrollgruppe ergaben sich keine Unterschiede in Bezug auf die kognitive Entwicklung (erfasst mit zwei Untertests des Standford Binet), wenn die Effekte der möglicherweise konfundierenden Variablen Alter, Geschlecht, ethnische Zugehörigkeit und sozioökonomischer Status durch Parallelisierung der Gruppen kontrolliert wurden. Als Kritikpunkt an dieser Studie ist aber die sehr kleine Gruppengröße (jeweils 20 Kinder) zu nennen. Im Gegensatz dazu wurde im Rahmen einer multizentrischen Studie in den USA (Messinger et al, 2004) eine sehr große Zahl an Untersuchungsteilnehmern rekrutiert und im Alter von 1, 2 und 3 Jahren untersucht. Kinder mit pränataler Kokainexposition $(\mathrm{N}=474)$ erreichten im Vergleich zu einer nach ethnischer Zugehörigkeit, Geschlecht und Gestationsalter parallelisierten Kontrollgruppe $(\mathrm{N}=655)$ einen geringeren Wert im Bayley Mental Development Index, nach Kontrolle des Geburtsgewichtes und Variablen $\mathrm{zu}$ Umwelt- und Erziehungsbedingungen (z.B. sozioökonomischer Status, Betreuungswechsel) war der Effekt nicht mehr signifikant.

Nair et al. (2003) fanden hingegen bei Kindern drogenmissbrauchender Mütter (Kokain oder Heroin) keinen Einfluss der Stärke der psychosozialen Risiken (z.B. mütterliche Depression, Gewalt, soziale Isolation) auf die kindliche Entwicklung (Bayley Mental and Motor Development Scales). Diese Aussage bezieht sich allerdings nur auf einen Vergleich innerhalb der Gruppe von Kindern substanzabhängiger Mütter und der Vergleich mit einer Kontrollgruppe fehlt.

\subsubsection{Effekte der Opiate}

Speziell mit den Auswirkungen des Opiatkonsums haben sich einige wenige Studien befasst: Bauman \& Levine (1986) untersuchten Kinder im Vorschulalter, deren Mütter methadonsubstituiert waren. Gegenüber einer Kontrollgruppe schnitten diese Kinder beim Standfort-Binet Intelligenztest signifikant schlechter ab. Wilson, McCreary, Kean \& Baxter (1979) haben schon früh ein gut durchdachtes, sowohl die biologischen als auch die psychosozialen Risikofaktoren berücksichtigendes Design verwendet, wodurch ihre Studie noch immer Aktualität besitzt. Eine Gruppe von 3-6jährigen Kindern heroinabhängiger Mütter wurde mit drei anderen Gruppen verglichen, die nach Alter, Geschlecht, ethnischer Zugehörigkeit, Geburtsgewicht und sozioökonomischen Status parallelisiert waren. Kinder der ersten Gruppe hatten ebenfalls heroinabhängige Mütter, waren aber keiner pränatalen Drogenexposition ausgesetzt, die Kinder der zweiten Gruppe gehörten einer Hoch-Risiko- 
Gruppe an (medizinische Komplikationen bei Geburt) und die Kinder der dritten Gruppe waren nach dem sozioökonomischen Status parallelisiert. Bezogen auf die sprachliche (Illinois Test of Psycholinguistic Abilities) und kognitive Entwicklung (McCarthy Scales of Children's Abilities) schnitten die Kinder mit pränataler Drogenexposition am schlechtesten ab, knapp gefolgt von den Kindern der ersten Vergleichsgruppe, deren Mütter heroinabhängig waren, bei denen aber keine pränatale Exposition bestand. Die beiden anderen Vergleichsgruppen erzielten signifikant bessere Ergebnisse als die beiden Gruppen in denen der prä- und postnatale mütterliche Drogenkonsum eine Rolle spielte.

In einer deutschen Studie (Bunikowski et al., 1998) erzielten die Kinder opiatabhängiger Mütter mit einem Alter von einem Jahr in den Griffiths-Entwicklungsskalen in der Skala Motorik und der Skala Leistung sowie im Gesamt-Entwicklungsquotienten ein signifikant niedrigeres Ergebnis als die Kontrollgruppe. Die opiatexponierten Kinder zeigten auch größere neurologische Auffälligkeiten als die Kontrollgruppe. Die Kontrollgruppe war hinsichtlich des Nikotinkonsums parallelisiert, andere wichtige Einflussfaktoren wie mütterliche Charakteristika und neonatale Daten unterschieden sich aber zum Teil deutlich in den beiden Gruppen, so dass nicht klar ist, ob es sich um direkte oder indirekte Effekte der Opiatexposition handelt.

Im Gegensatz zu den oben genannten Ergebnissen konnten Kaltenbach \& Finnegan (1987) im Alter von 6 Monaten bei einer Gruppe von Kindern mit in-utero-Methadon-Exposition im Vergleich zu einer Gruppe von Kindern ohne Drogenexposition in den Bayley Mental Development Scales keinen Unterschied in der kognitiven Entwicklung finden. Die Gruppen waren nach ethnischer Zugehörigkeit, Alter der Mutter und sozioökonomischem Status parallelisiert. Eine Rolle spielen könnte hier das Alter der Kinder, da, wie weiter oben ausgeführt, sich Defizite umso mehr zeigen, je älter die Kinder zum Zeitpunkt der Untersuchung sind. De Cubas \& Field (1993) verglichen Kinder methadonsubstituierter Mütter mit Kindern einer Kontrollgruppe, wobei die Kontrollgruppe in dieser Studie nicht nur nach demographischen Variablen parallelisiert war, sondern auch die medizinischen Probleme nach der Geburt in beiden Gruppen vergleichbar waren. Zum Untersuchungszeitpunkt, in einem Alter von 6-13 Jahren, zeigten sich im Standford Binet Intelligenztest und in der KABC (Kaufman Assessment Battery for Children) keine Unterschiede zwischen den Gruppen, allerdings erreichte die Subgruppe der Kinder mit Entzugssymptomen ein signifikant niedrigeres Ergebnis im Stanford Binet als die Kinder ohne Entzugssymptome. Die Bedeutung des neonatalen Abstinenzsyndroms als Risikofaktor für die spätere Entwicklung 
haben auch Berger und Elstner (2002) hervorgehoben, van Baar \& de Graaff (1994) fanden aber im Gegensatz dazu keine Assoziation zwischen Stärke der Entzugssymptomatik (operationalisiert durch die Behandlungsdauer) und kognitiver Entwicklung.

Hans und Jeremy (2001) fanden in ihrer Untersuchung an Kindern opiatabhängiger, methadonsubstituierter Mütter im Alter von 2 Jahren, dass diese bei den Bayley Skalen zwar eine Leistung erbrachten, die noch im Normbereich lag, im Vergleich zu einer Gruppe von Kontrollkindern war sie allerdings signifikant schlechter. Bei der Analyse relevanter Kovariaten zeigte sich, dass der mütterliche Opiatkonsum weniger einen direkten Einfluss auf die Entwicklung hat, sondern, dass die niedrige Leistung in der kognitiven Entwicklung mit dem hohen psychosozialen Risiko assoziiert war und die schlechtere psychomotorische Entwicklung vor allem durch das geringere Geburtsgewicht in der Gruppe der Kinder der substituierten Mütter bedingt war. Viele der erhobenen psychosozialen Risikofaktoren wie etwa die mütterliche Schulbildung und der sozioökonomische Status waren auch in der Kontrollgruppe ähnlich niedrig, es zeigte sich aber, dass das Ausmaß der mütterlichen Psychopathologie und des inadäquaten Erziehungsverhaltens in der Gruppe der substituierten Mütter besonders hoch war. Die Entwicklungsauffälligkeiten sind dieser Studie nach also vor allem als indirekte Effekte der prä- und postnatalen Drogenexpositon zu interpretieren. $\mathrm{Zu}$ ganz ähnlichen Ergebnissen kamen Messinger et al. (2004), die in ihrer multizentrischen Studie neben einer Gruppe mit Kokainexposition auch eine Gruppe von Kindern mit pränataler Opiatexposition $(\mathrm{N}=50)$ mit einer sehr großen $(\mathrm{N}=655)$ und nach ethnischer Zugehörigkeit, Geschlecht und Gestationsalter parallelisierten Kontrollgruppe verglich. Die Kinder der Opiatgruppe erreichten im Bayley Psychomotor Development Score zwar geringere Werte als die Vergleichsgruppe nicht exponierter Kinder, bei Kontrolle relevanter Kovariaten (geringes Geburtsgewicht, psychosoziale Umstände, mütterlicher PPVT-Score) waren die gefundenen Effekte nicht mehr signifikant.

\subsection{Sozial-emotionale Entwicklung}

Kandel (1990) konnte zeigen, dass in Abhängigkeit vom Schweregrad des Substanzkonsums der Eltern zum Zeitpunkt der Untersuchung bei den Kindern größere Verhaltensauffälligkeiten vorliegen (das Ausmaß der Drogenproblematik war dabei folgendermaßen definiert: 0 = weder legale, illegale Drogen noch Medikamentenmissbrauch; $1=$ nur Gebrauch von Zigaretten oder Alkohol; 2 = Gebrauch von Marihuana aber keiner 
anderen illegalen Drogen; 3 = Gebrauch illegaler Drogen; $4=$ illegale Drogen und Medikamentenmissbrauch). Je größer der Drogenkonsum, desto eher wurden die Kinder, die zum Untersuchungszeitpunkt 6 Jahre oder älter waren, von ihren Müttern als aggressiv, zurückgezogen, wenig ausgeglichen und wenig folgsam beschrieben. Dieser Effekt der Stärke des mütterlichen Substanzkonsums blieb auch nach Kontrolle anderer Variablen, wie mütterlicher Schulbildung und Alter des Kindes, signifikant. Die Auswirkungen des mütterlichen Substanzkonsums auf das Verhalten der Kinder ist in dieser Studie als Effekt der Erziehungsbedingungen und der psychosozialen Umstände $\mathrm{zu}$ interpretieren, der Drogenkonsum der Mütter zum Zeitpunkt von Schwangerschaft und Geburt wurde nicht erhoben.

Martin et al. (1994) kamen in ihrer Studie über externalisierendes Problemverhalten in der Kindheit zu dem Schluss, dass Jungen mit einer Familiengeschichte von Substanzmissbrauch (Drogen oder Alkohol) signifikant mehr Aggressivität, Aufmerksamkeitsprobleme und Impulsivität aufweisen. Bezüglich der Hyperaktivität fanden sich keine Unterschiede zu einer Kontrollgruppe von Jungen ohne Substanzmissbrauch in der Familie. Die Zuordnung zu den Gruppen erfolgte dabei über den Substanzmissbrauch des Vaters, d.h. die gefundenen Effekte sind ebenso wie bei Kandel vor allem als Einfluss des Milieus zu interpretieren, obwohl eine biologische Vulnerabilität durch eine mögliche Substanzabhängigkeit der Mutter und eine Drogenexposition in-utero nicht ausgeschlossen werden kann.

Wilens et al. (1995) untersuchten eine Gruppe von 4-18jährigen Kindern und Jugendlichen, deren Mütter oder Väter opiatabhängig und methadonsubstituiert waren. Die Kinder der Experimentalgruppe zeigten im Vergleich zu einer Kontrollgruppe in der Child Behavior Checklist (CBCL), einem Verfahren zur Einschätzung der Psychopathologie, signifikant niedrigere Werte in den Kompetenzskalen und höhere Werte in den Gesamtskalen Internalisierend und Externalisierend. Ebenfalls erhöht waren die Skalen Dissoziales Verhalten, Schizoid/Zwanghaft und Aufmerksamkeitsprobleme, allerdings zeigte eine Gruppe von Kindern mit der Diagnose ADHD eine noch größere Auffälligkeit. Auch hier lässt sich durch das Studiendesign nicht beantworten, ob das erhöhte Psychopathologierisiko eher auf die Umweltbedingungen oder auf biologische Einflüsse zurückzuführen ist.

De Cubas \& Field (1993) ermittelten, dass Kinder methadonsubstituierter Mütter im Alter von 6-13 Jahren im Vergleich zu einer gut parallelisierten Kontrollgruppe in der CBCL in den Skalen Angst/Depression, Sozialer Rückzug, Somatische Beschwerden, Hyperaktivität, Aggression, Dissoziales Verhalten, Internalisierend und Externalisierend als auffälliger 
beschrieben wurden. Griffith, Azuma \& Chasnoff (1994) kamen bei ihrem Vergleich zwischen Kindern kokainabhängiger Mütter ohne zusätzlichen Opiatkonsum (Gruppe 1), Kindern substanzmissbrauchender Mütter ohne Kokain (Marihuana, Alkohol und Opiate, Gruppe 2) sowie einer Kontrollgruppe ohne Drogenkonsum (Gruppe 3) $\mathrm{zu}$ folgendem Ergebnis: Im Urteil der Bezugspersonen in der CBCL, wurden die Kinder der beiden Gruppen mit Drogenexposition als aggressiver eingeschätzt als die Kinder der Kontrollgruppe und im Expertenurteil wurde bei ihnen eine geringere Fähigkeit zur Aufrechterhaltung der Aufmerksamkeit während einer schwierigen Aufgabe beschrieben.

Richardson (1998) fanden deutliche Unterschiede im Temperament und Verhalten zwischen Kindern von Müttern, die während des 1. Trimesters Kokain konsumiert hatten und Kindern einer Kontrollgruppe. In einer longitudinalen Studie waren die Kinder aus der ersten Gruppe im Alter von einem Jahr auf der von den Müttern eingeschätzten Bates Infant Characteristics Scale in der Skala „fussy/difficult“ auffälliger. Die Kinder zeigten laut dem Urteil der Untersucher auch eine geringere Responsivität und reagierten weniger auf die Testmaterialen. Ihre Aufmerksamkeitsspanne wurde als kürzer eingeschätzt und die motorische Unruhe während der Testung war erhöht. Richardson interpretierte diese Ergebnisse als Beleg der teratogenen Wirkung von Kokain.

Einige der ganz wenigen Studien, in denen die Erfassung der Psychopathologie auf der Einschätzung der Lehrer beruht, stammt von Delaney-Black et al. (2000). Insgesamt wurden 471 Kinder zum Zeitpunkt des Besuchs der ersten Klasse untersucht, bei 201 von ihnen hatte pränatal eine Kokainexposition bestanden. Die Kinder mit Kokainexposition wiesen eine signifikant höhere Differenz der CBCL-Skalen Externalisierend-Internalisierend auf und die Jungen mit pränataler Kokain-Exposition hatten gegenüber den Kindern der Kontrollgruppe ein zweifach erhöhtes Risiko, in den Skalen Externalisierend und Dissoziales Verhalten einen Wert im klinisch auffälligen Bereich zu erreichen. Als ein weiteres Resultat zeigte sich, dass die Kinder der Experimentalgruppe, die einen Bezugspersonenwechsel erlebt hatten und aktuell wieder bei der Mutter lebten, in den Skalen Externalisierend und im Differenzscore Externalisierend-Internalisierend signifikant höhere Werte erreichten als die Kontrollgruppe. Als Einschränkung bezüglich der Generalisierbarkeit der Ergebnisse ist zu nennen, dass die Mütter in dieser Studie alle an einer Schwangerschaftsvorsorge teilnahmen, was sicher nicht auf die Gesamtgruppe der drogenabhängigen Mütter zutrifft und mögliche Effekte eher minimiert. 
Phelps et al. (1997) ermittelten in ihrer Untersuchung, dass die Gruppe der 3-6jährigen Kinder mit einer Kokain/Polydrogen-Exposition in-utero in einem von der Mutter ausgefüllten Fragebogen zu den sozialen Fähigkeiten (SSRS) sowie in den CBCL-Skalen Internalisierend und Externalisierend Werte erreichten, die durchschnittlich eine Standardabweichung unter dem erwarteten Mittelwert lagen. Da dies aber nicht schlechter war als die Ergebnisse der nach Alter, ethnischer Zugehörigkeit, Geschlecht und sozioökonomischem Status parallelisierte Kontrollgruppe, wurde das nicht als direkter Einfluss der in-uteroDrogenexposition gewertet, sondern den Umweltbedingungen zugeschrieben.

\subsection{Entwicklung unter dem Aspekt unterschiedlicher}

\section{Erziehungsbedingungen}

In vielen Studien, wie auch in einigen der oben genannten Untersuchungen zur sozialemotionalen Entwicklung, lassen sich Unterschiede in der Entwicklung nicht eindeutig auf biologische oder Umwelteffekte zurückführen, und auch wenn mittlerweile viele Forschungsgruppen Charakteristika der Kinder und Mütter als Moderator-Variablen berücksichtigen, gibt es weiterhin einen Mangel an Studien, die in ihrem Studiendesign neben der biologischen Vulnerabilität speziell auf die postnatalen Erziehungsbedingungen und den für Kinder drogenabhängiger Eltern so prominenten Faktor Fremdbetreuung und Bezugspersonenwechsel fokussieren.

$\mathrm{Zu}$ den Auswirkungen unterschiedlicher Varianten von Fremdbetreuung konnten Frank et al. (2002) einen Beitrag leisten. Bei Kindern bis zu zwei Jahren konnte zwar keine direkte Auswirkung der Kokainexposition auf den Bayley Mental and Psychomotor Index festgestellt werden, im Vergleich zu bei den leiblichen Müttern lebenden Kindern wurden aber bei Kindern, die fremdplatziert worden waren folgende Effekte beschrieben: Kindern in Verwandtenpflege erzielten im MDI im Alter von 6 Monaten signifikant schlechtere Ergebnisse als Kinder bei leiblichen Müttern; im Alter von 24 Monaten war die Leistung hingegen angeglichen. Anders allerdings bei den in Pflegefamilien ohne Verwandtschaftsverhältnis aufgenommenen Kindern: Im Alter von 6 Monaten war der MDIIndex ebenfalls signifikant niedriger, während er im Alter von 24 Monaten signifikant höher war. Fazit dieser Studie ist demnach eine möglicherweise protektive Wirkung nichtverwandtschaftlicher Pflegeverhältnisse auf die kognitive Entwicklung - eine von den Autoren angeführte mögliche Erklärung ist dabei die in den USA geringere Unterstützung der 
Verwandtenpflege durch den Staat, sowohl finanziell als auch durch öffentliche soziale Einrichtungen. $\mathrm{Zu}$ ähnlichen Ergebnissen waren bereits Tyler et al. (1997) gekommen: Im Alter von 6 Monaten war das Ergebnis im Bayley MDI bei Kindern in Verwandtenpflege signifikant schlechter als bei den bei der Mutter verbliebenen Kindern. Diese Ergebnisse sind aufgrund des Alters der Kinder nicht ganz leicht zu erklären, da Ergebnisse von Entwicklungstests im Alter von 6 Monaten eher die biologischen Bedingungen widerspiegeln; die Einflüsse der Erziehung und Umweltbedingungen schlagen erst später höher zu Buche. Ähnlich wie Frank et al. (2002) vermuten aber auch Tyler et al. (1997), dass das Setting der Verwandtenpflege spezifische Risiken für die Entwicklung der Kinder in sich birgt. Dazu gehört die anhaltende psychische Belastung, welche die Verwandten, meist die Großeltern, im Umgang mit ihren erwachsenen drogensüchtigen Kindern erleben sowie das deutlich höhere Alter der Großeltern.

Brown, Bakeman, Coles, Platzman \& Lynch (2004) konnten in ihrer teilweise auch explorativ angelegten Untersuchung in Bezug auf die sprachliche und kognitive als auch die sozialemotionale Entwicklung bei zweijährigen Kindern keine direkten Effekte der Kokainexposition in-utero finden. Wurde die Gruppe der Kinder drogenabhängiger Mütter allerdings unterteilt in Kinder, die bei ihren Eltern aufwachsen, und Kinder, die fremdplatziert worden waren, so zeigte sich, dass die Kinder der zweiten Gruppe in Bezug auf die kognitive Entwicklung (Bayley Mental Development Index) und die Psychopathologie bzw. die sozialemotionale Entwicklung (CBCL) besser abschnitten als die Kinder, die bei ihren Eltern verblieben waren. Innerhalb der Gruppe der fremdplatzierten Kinder ergaben sich ebenfalls Hinweise, dass die Nichtverwandtenpflege der Entwicklung der Kinder förderlicher ist als die Verwandtenpflege.

In eine ganz ähnliche Richtung gehen die Ergebnisse einer groß angelegten Studie (Ornoy, Michailevskaya, Lukashov, Bar-Hamburger \& Harel, 1996). Kinder von heroinsüchtigen Müttern wurden verglichen mit einer Gruppe von Kindern heroinabhängiger Väter, einer Gruppe emotional und physisch vernachlässigter Kinder sowie zwei Kontrollgruppen mit Kindern aus mittleren bis guten sozioökonomischen Verhältnissen und gesunden Kindern aus einem Kindergarten. Die niedrigsten IQ-Werte erzielte dabei die Gruppe der vernachlässigten Kinder. Die Kinder heroinabhängiger Väter erzielten ein (wenn auch nicht signifikant) niedrigeres Ergebnis als die Gruppe der Kinder heroinabhängiger Mütter. Wurde die letztgenannte Gruppe unterteilt in bei den Müttern verbliebenen Kinder und kurz nach der Geburt adoptierten Kinder, dann schnitten die adoptierten Kinder nicht schlechter ab als die 
Kontrollgruppen, während die bei den Müttern lebenden Kinder nun mit den Kindern der drogenabhängigen Väter vergleichbar waren. Kinder opiatabhängiger Mütter haben demnach also ein „,normales“ Entwicklungspotential, vorausgesetzt die Entwicklungsbedingungen sind optimal. Zu dem gleichen Schluss kamen van Baar \& de Graaff (1994): Pränatal drogenexponierte (Opiate und/oder Kokain) Kinder, die bei Pflegeeltern aufwuchsen, näherten sich mit der Zeit immer mehr den Intelligenztestleistungen einer Kontrollgruppe an; im Alter von fünfeinhalb Jahren waren die Unterschiede nicht mehr signifikant. In einem adäquaten Umfeld hatte somit ein „Catch-up“ im kognitiven Leistungsvermögen stattgefunden.

Moe und Slinning (2001) setzten sich in ihrer Studie an Kindern, deren Mütter Kombinationen verschiedener Drogen (Cannabis, Amphetamine), als Hauptdroge aber Heroin konsumiert hatten, das Ziel, die Entwicklung der Kinder unter adäquaten Erziehungsbedingungen zu verfolgen. Der Anteil der kurz nach der Geburt bei Adoptiv- oder Pflegeeltern untergebrachten Kinder war mit 84,6\% dementsprechend hoch. Adoptiv/Pflegeeltern und die Eltern der Kontrollgruppe waren dem sozioökonomischen Status und der Schulbildung nach parallelisiert. Im Bayley Mental Development Index erzielten die drogenexponierten Jungen, nicht aber Mädchen, im Alter von 1, 2 und 3 Jahren signifikant niedrigere Ergebnisse und dieser Gruppe x Geschlechts Effekt blieb auch nach Kontrolle des sozioökonomischen Status und des Gestationsalters signifikant. Da die Erziehungsbedingungen zu einem gewissen Grad optimiert waren, sprechen die Ergebnisse dafür, dass pränataler Drogenkonsum, zumindest bezogen auf Jungen, zu einer Vulnerabilität für die weitere Entwicklung führt. Interessanterweise kam es innerhalb der drogenexponierten Gruppe über den Zeitraum von 3 Jahren zu einem Aufholen der Entwicklungsrückstände, was, wie die Autoren meinen, wahrscheinlich durch die kompensierende Wirkung der optimierten Erziehungsbedingungen bedingt war.

Dass die Möglichkeiten der Kompensation aber nicht unbegrenzt sind, konnten Simmel, Brooks, Barth und Hinshaw (2001) in ihrer Langzeitstudie an Adoptivkindern in den USA belegen. Es fanden sich eindeutige Assoziationen zwischen externalisierendem Verhalten (erhoben im Alter von 4-18 Jahren) und Risikofaktoren, die vor der Adoption wirksam waren. Dazu gehörten die pränatale Drogen- und Alkoholexposition, Missbrauch und Vernachlässigung durch die leiblichen Eltern, ein späteres Adoptionsalter und der Aufenthalt in mehreren Heimen oder Pflegestellen vor der endgültigen Adoption. 
Wie kritisch der Zeitpunkt der Adoption ist, konnten in Bezug auf Kinder drogenabhängiger Eltern auch Berger und Elstner (2002) zeigen: Kinder mit einem Betreuungswechsel im 1. Lebensjahr zeigten eine bessere Entwicklung als Kinder mit einem Betreuungswechsel im 2. Lebensjahr. Diese Ergebnisse decken sich mit bisherigen Erkenntnissen, dass Kinder besonders in der Zeit zwischen dem 6. und 24. Lebensmonat, in einem Zeitraum, in dem stabile Bindungen aufgebaut werden, eine große Vulnerabilität für Beziehungsabbrüche und Fremdunterbringung aufweisen (Rutter, 1987; Yarrow, 1964). Die Auswirkungen erlebter Deprivation in Abhängigkeit von ihrer Dauer belegten Rutter et al. (1998) in der ERA Studie: Bei der Untersuchung rumänischer Waisenkinder, die nach dem Ende des Ceaucescu Regimes nach England adoptiert worden waren, zeigte sich zunächst eine deutliche körperliche als auch kognitive Retardierung der rumänischen Waisenkinder. Bis zum nächsten Untersuchungszeitpunkt im Alter von 4 Jahren mit den Denver Skalen und McCarthy Skalen war die Entwicklung der rumänischen Kinder, die vor einem Alter von 6 Monaten nach England kamen, mehr oder weniger vergleichbar mit der Entwicklung der Vergleichsgruppe englischer Adoptivkinder. Die Kinder, die später in einem Alter zwischen 6 und 24 Monaten adoptiert wurden, zeigten ebenfalls einen sehr deutlichen Aufholeffekt hinsichtlich ihrer Entwicklung, blieben allerdings mit durchschnittlich mehr als einer Standardabweichung signifikant hinter den englischen und vor einem Alter von 6 Monaten adoptierten rumänischen Waisenkindern zurück. Eine frühe Adoption erwies sich damit als prognostisch günstiger Faktor für die weitere Entwicklung. 


\subsection{Zusammenfassung bisheriger Forschungsergebnisse}

Es gehört zu den unumstrittenen Tatsachen, dass Kinder aus suchtbelasteten Familien im Allgemeinen und Kinder mit einer pränatalen Drogenexposition im Speziellen einer Vielzahl von medizinischen und psychosozialen Risiken ausgesetzt sind. Breiter Konsens herrscht auch darüber, dass die psychosozialen Begleiterscheinungen des elterlichen Drogenkonsums im Sinne einer Hoch-Risiko-Umwelt die kognitive und sozial-emotionale Entwicklung negativ beeinflussen. Wie die Interaktion dieser Risiken und speziell die Rolle einer biologischen Vulnerabilität auf die kindliche Entwicklung einwirken, darüber existieren hingegen unterschiedliche und teilweise einander widersprechende Ergebnisse.

Hinsichtlich der kognitiven Entwicklung ermittelten mehrere Forschergruppen sowohl in Bezug auf Kokainexposition als auch Opiatexposition erniedrigte Werte in Skalen zur kognitiven, sprachlichen oder psychomotorischen Entwicklung, teilweise verschwindet aber der Gruppeneffekt, sobald demographische, körperliche (z.B. Geburtsgewicht) oder auch Variablen des familiären Umfelds miteinbezogen werden und lassen die Beeinträchtigung der drogenexponierten Kinder als rein durch Umwelteinflüsse bzw. durch unspezifische somatische Risikovariablen (Geburtsgewicht) bedingt erscheinen. Für einen biologischen Effekt sprechen Ergebnisse, die eine eindeutige Beziehung zwischen Höhe der Dosierung bzw. speziell für die Opiatexposition zwischen Schwere der Entzugssymptomatik und Entwicklungsbeeinträchtigung ermitteln.

Zur sozial-emotionalen Entwicklung gibt es weit weniger Befunde, relativ einheitlich wird bei Kindern drogenabhängiger Eltern aber eine erhöhte Psychopathologie beschrieben, wobei besonders externalisierendes Verhalten und Aufmerksamkeitsprobleme hervorzuheben sind. In diesem Zusammenhang können Befunde (Richardson, 1998), die bei einjährigen Kindern eine geringere Responsivität, eine kürzere Aufmerksamkeitsspanne und eine erhöhte motorische Unruhe festgestellt haben, als Vorboten der später beobachteten Verhaltensauffälligkeiten interpretiert werden.

Um die Effekte der pränatalen Drogenexposition von den Effekten suboptimaler Erziehungsbedingungen $\mathrm{zu}$ trennen, wurden mehrere Adoptionsstudien durchgeführt. Ein überraschend einheitliches Ergebnis ist der positive Effekt nichtverwandtschaftlicher gegenüber verwandtschaftlicher Pflegeverhältnissen auf die kindliche Entwicklung, wobei allerdings fraglich ist, inwieweit diese Ergebnisse aus dem amerikanischen Raum auf 
deutsche Verhältnisse übertragbar sind. Generell sprechen die Forschungen zur Auswirkung unterschiedlicher Erziehungsbedingungen deutlich für das transaktionale Modell, wonach eine potentiell vorhandene biologische Vulnerabilität gänzlich oder zu einem großen Teil durch eine Optimierung der Erziehungsbedingungen kompensiert werden kann. 


\section{Hintergrund und Ziel der aktuellen Untersuchung}

Diese Arbeit ist Teil eines insgesamt sechsjährigen, von der Deutschen Forschungsgesellschaft (DFG) finanzierten Projektes zur Untersuchung der Wirkung und Wechselwirkung risikobehafteter und protektiver Faktoren auf eine mögliche spätere Suchtentwicklung bei Kindern und Jugendlichen aus einer Hochrisikogruppe („Studie an einer Hochrisikogruppe mit neonatalem Abstinenzsyndrom: Präkursoren und protektive Merkmale der Suchtentwicklung“). Die DFG-Studie ist als prospektive Längsschnittuntersuchung konzipiert und baut auf der Arbeit von Ziegler (1998) auf, der aus den Krankenakten der Abteilung für Neonatologie des Universitätsklinikums der Johann Wolfgang Goethe-Universität Frankfurt am Main alle Fälle von neonatalem Abstinenzsyndrom (NAS) aus den Geburtsjahrgängen 1988 bis 1995 retrospektiv erfasst hat. Diese Stichprobe wird im Folgenden als Ziegler-Stichprobe bezeichnet. Die Arbeit von Ziegler zeigt den Ist-Zustand der auf Kinder opiatabhängiger Mütter einwirkenden postpartalen Risikofaktoren, d.h. die Entzugssymptomatik, die sozioökonomische Situation der Familie und die Fürsorgequalitäten der Eltern, und dient als Ausgangspunkt für die Erforschung der weiteren Entwicklung dieser Hochrisikokinder.

Zusätzlich zur Hochrisikogruppe wurde aus den neonatologischen Stationen des Universitätsklinikums aus denselben Geburtsjahrgängen eine nach Alter der Mutter, Parität und sozioökonomischen Status parallelisierte Vergleichsstichprobe erhoben. Die Kinder dieser Gruppe wurden aufgrund medizinischer Komplikationen behandelt, wiesen aber keine Entzugssymptomatik bzw. Drogenexposition auf. Eine solche Vergleichsgruppe ermöglicht die Absicherung der Spezifität der Ergebnisse, denn bei einer aus gesunden Säuglingen zusammengesetzten Kontrollgruppe besteht die Gefahr, dass festgestellte Unterschiede in der Entwicklung von Kindern opiatabhängiger Mütter und den Kindern der Vergleichsgruppe auf vom Opiatkonsum unabhängigen Risikofaktoren, wie etwa Frühgeburtlichkeit oder andere medizinischer Risiken, zurückzuführen sind.

Die vorliegende Arbeit beruht auf den innerhalb dieser prospektiven Studie erhobenen Daten der ersten drei Jahre (11/2000 bis 11/2003). Während das Hauptaugenmerk des DFGForschungsprojektes auf einer potentiellen späteren Suchtentwicklung dieser Kinder liegt, ist das Hauptinteresse dieser Studie die kognitive und die sozial-emotionale Entwicklung der Kinder drogenabhängiger Mütter unter Berücksichtigung verschiedener Einflussfaktoren 
(Risiko- und Schutzfaktoren). Der Ausdruck „sozial-emotionale“ bzw. „emotionale“ Entwicklung bezieht sich in dieser Arbeit auf die Psychopathologie des Kindes sowie auf dessen psychosoziales Funktionsniveau. 


\section{Hypothesen und Fragen}

In der vorliegenden Untersuchung soll die kognitive und sozial-emotionale Entwicklung von Kindern ab einem Alter von 6 Jahren, bei denen eine pränatale Opiatexposition bestand, untersucht werden. Dabei werden gemäß des transaktionalen Modells sowohl das biologische Risiko als auch die postnatal bestehenden Umweltfaktoren berïcksichtigt. Genützt werden dabei die Vorteile des späten Nachuntersuchungszeitpunktes und die damit verbundene natürlichen Gruppierung der Kinder in drei Gruppen verschiedener Betreuungsverhältnisse: Kinder, die seit ihrer Geburt bei ihren leiblichen Eltern geblieben sind, Kinder, die innerhalb kurzer Zeit nach der Geburt in eine Fremdbetreuung vermittelt wurden, und Kinder, die erst nach längerer Zeit oder nach mehreren Betreuungswechseln in ihr derzeitiges Betreuungsverhältnis vermittelt wurden. Diese Einteilung schafft Gruppen mit unterschiedlicher Ausprägung und Qualität der psychosozialen Risiken. Während man in der Gruppe der unmittelbar nach der Geburt zu Pflege- oder Adoptiveltern vermittelten Kinder von relativ optimalen Erziehungsbedingungen ausgehen kann, stellt die Gruppe der Kinder mit spätem oder mehrmaligem Bezugspersonenwechsel sicher die Gruppe mit besonders ungünstigen psychosozialen Bedingungen dar. Die Gruppe der bei den leiblichen Eltern verbliebenen Kinder hat demgegenüber zwar keinen Wechsel der Bezugspersonen zu verkraften, sie sind aber anhaltend den für das Drogenmilieu spezifischen psychosozialen Gefährdungen ausgesetzt.

Als Hypothesen werden deshalb formuliert:

I. Kinder, die spät fremdplatziert wurden bzw. mehrere Wechsel erlebt haben, weisen die stärkste psychosoziale Belastung auf. Die geringste psychosoziale Belastung besteht bei den früh fremdplatzierten Kindern.

Spezifisch in Bezug auf die Gruppe opiatexponierter Kinder wird basierend auf der oben beschriebenen Literatur folgende Hypothese formuliert:

II. Zwischen der Schwere des Neonatalen Abstinenzsyndroms, als Indikator des pränatalen biologischen Risikos, und der kognitiven Entwicklung besteht ein Zusammenhang. 
Aufbauend auf der oben diskutierten Literatur wird davon ausgegangen, dass bei Kindern mit in-utero-Exposition eine erhöhte Vulnerabilität für Auffälligkeiten in der kognitiven und emotionalen Entwicklung vorliegt, die aber durch die postnatalen Betreuungsbedingungen und den davon abhängigen psychosozialen Bedingungen (Hypothese I) kompensiert, abgemildert oder verstärkt werden können.

Als weitere Hypothesen werden formuliert:

III. Kinder drogenkonsumierender Eltern, die erst spät bzw. auch mehrere Betreuungswechsel erlebt haben, weisen die stärkste Beeinträchtigung in Bezug auf die weitere Entwicklung auf, da bei ihnen die biologischen und die psychosozialen Risikofaktoren zusammenwirken.

IV. Kinder, die bereits kurz nach der Geburt fremdbetreut wurden, weisen trotz biologischer Vulnerabilität eine mit den Kindern der Kontrollgruppe vergleichbare kognitive und emotionale Entwicklung auf. 


\section{Methode}

\subsection{Stichprobenrekrutierung}

\subsubsection{Die Stichprobe der aktuellen Untersuchung}

Von der 151 Kinder (Gruppe der Kinder opiatabhängiger Mütter, Kinder mit neonatalem Abstinenzsyndrom (NAS) $\mathrm{N}=101$; Kontrollgruppe $\mathrm{N}=50$ ) umfassenden ZieglerGesamtstichprobe konnten $\mathrm{N}=65$ Kinder in unserer Klinik oder bei Hausbesuchen untersucht werden; drei Kinder wurden durch Hinweise des Jugendamtes (Pflege- und Adoptivkinder) zusätzlich rekrutiert. Bei 10 Kindern waren die Erziehungsberechtigen zwar nicht zur Teilnahme an der Untersuchung, wohl aber zum Ausfüllen eines Fragebogens bereit, so dass von insgesamt 78 Kindern Daten vorliegen. Von diesen 78 Kindern sind 57 zur Experimentalgruppe (EG) (aus dem Kollektiv der Gruppe der Kinder mit neonatalem Abstinenzsyndrom; NAS) zu zählen, 50 davon sind untersucht, von sieben Kindern liegen Fragebögen vor. 21 Kinder (davon 18 untersucht, von 3 liegen Fragebögen vor) stammen aus dem Kollektiv der Vergleichsgruppe (Kontrollgruppe von Ziegler) und bilden in der vorliegenden Studie die Kontrollgruppe (KG). Aus jeder der ursprünglich von Ziegler rekrutierten Gruppe konnte also ca. mit der Hälfte Kontakt aufgenommen werden.

\subsubsection{Schwierigkeiten bei der Rekrutierung}

Die letztendlich erreichte Zahl an Untersuchungsteilnehmern ist unter den gegebenen Voraussetzungen, wie unter anderem Zugehörigkeit zum Drogenmilieu, schwer auffindbare Familien und mangelndes Interesse an der Teilnahme, beachtlich, und war mit großem Aufwand verbunden. In einem hierarchischen Vorgehen wurden mehrere Schritte unternommen, um die Kinder und ihre Familien aufzufinden. Bei der Mehrzahl der Kinder stimmten die aus den Krankenakten vermerkten Adressen nicht mehr, so dass für alle Familien eine Meldeamtsuche über den Namen der Mutter und später auch über den Namen des Kindes durchgeführt wurde. Da ein großer Teil der Kinder nicht mehr bei den leiblichen Eltern lebte, wurden die Frankfurter Sozialrathäuser hinzugezogen, welche für die 
Pflegekinder innerhalb Frankfurts zuständig sind, sowie der „Besondere Dienst Jugendhilfe“, dem die Vermittlung von Pflegekindern außerhalb Frankfurts sowie von Adoptivkindern obliegt. Einige wenige Kinder konnten durch Unterstützung des Landesarbeitsamtes Hessen, Familienkasse, gefunden werden, da die Mütter Bezieher von Kindergeld sind. Besonders im Hinblick auf methadonsubstituierte Mütter wurden Erwachsenenpsychiater sowie das Gesundheitsamt um Mithilfe gebeten. Viele Informationen zur Auffindung von Müttern und Kindern kamen auch aus dem „Projekt Lichtblick“ der Integrativen Drogenhilfe e.V. sowie der HIV-Ambulanz der Universität Frankfurt/Main. In allen Fällen der Kontaktaufnahme wurden die ethischen und datenschutzrechtlichen Bestimmungen gewahrt, d.h. die vermittelnde Institution gab nicht die Adressen an unsere Klinik weiter, sondern es wurde ein Anschreiben unserer Klinik an die jeweilige Familie weitergeleitet. Um eine Familie bzw. ein Kind aufzufinden, waren im Allgemeinen mehrere Versuche über verschiedene Stellen notwendig, bzw. wurden die Familien teilweise auch mehrmals kontaktiert. Die Zahl nicht wahrgenommener Untersuchungstermine war erheblich und in einigen Fällen kam es bis zu vier Terminvereinbarungen, bevor tatsächlich ein Kontakt zustande kam. Aus Tabelle 8.1 sind die Anzahl der aus der ursprünglichen Stichprobe rekrutierten Kinder sowie die Gründe für eine Nichtteilnahme ersichtlich.

Tab. 8.1 Auflistung der Stichprobe

\begin{tabular}{|l|c|}
\hline Gesamtstichprobe von Ziegler & 151Kinder \\
\hline Davon Untersuchung in Klinik oder Hausbesuch & 65 \\
\hline Zusätzliche Rekrutierungen & 3 \\
\hline Nur Fragebögen vorhanden & $\mathbf{7 8 ~ K i n d e r n ~}$ \\
\hline Daten vorhanden von & 23 \\
\hline Familien bzw. Kinder nicht gefunden & 32 \\
\hline $\begin{array}{l}\text { Mit Sicherheit oder großer Wahrscheinlichkeit gefunden, } \\
\text { aber kein Interesse, bzw. keine Rückmeldung }\end{array}$ & 7 \\
\hline Über Institution kontaktiert, aber keine Bereitschaft zur Teilnahme & 4 \\
\hline Kind verstorben & 3 \\
\hline Kind im Ausland & 7 \\
\hline Sonstige Gründe (z.B. Heimunterbringung weit entfernt oder starke Behinderung) & \\
\hline
\end{tabular}




\subsection{Untersuchungsteilnehmer}

\subsubsection{Beschreibung der Ausgangsstichprobe}

Zunächst wird die Ausgangsstichprobe (Stichprobe von Ziegler) anhand ihrer medizinischen Daten genauer dargestellt. Wie weiter oben beschrieben handelte es sich bei dieser Ausgangsstichprobe um ein unausgelesenes Kollektiv, in dem alle Kinder, die in den Jahren 1988-1995 in der Universitätsklinik Frankfurt am Main geboren wurden und bei denen eine pränatale Opiatexposition festgestellt wurde, beinhaltet waren. Um sicherzustellen, dass die aktuelle Stichprobe in ihren medizinischen Daten der ursprünglichen entspricht und damit repräsentativ für das Drogenmilieu einer deutschen Großstadt ist, wurden, sofern dies möglich war, die Daten der Ausgangsstichprobe und die Daten der aktuellen Stichprobe miteinander verglichen. Alle angegebenen Daten beruhen auf der Dokumentation von Ziegler (1998).

\subsubsection{Substanzeinnahme}

Als Einschlusskriterium für die Experimentalgruppe von Ziegler (NAS) galt die Opiatabhängigkeit zu einem Zeitpunkt der Schwangerschaft. Opiatabhängigkeit der Mütter konnte dabei sowohl Heroinabhängigkeit und/oder Methadonsubstitution bedeuten. Da rein opiatabhängige Mütter in der Praxis selten zu finden sind, war der Beikonsum anderer Drogen kein Hindernis für den Einschluss in die Stichprobe. Eine genaue Erfassung der zusätzlich konsumierten Substanzen gestaltete sich sehr schwierig. In der von Ziegler erfassten Stichprobe wurde nur in 10-15\% der Fälle der Beikonsum anderer Substanzen in den Akten dokumentiert; ein Urinscreening wurde nur bei 29 der opiatabhängigen Mütter $(28,7 \%)$ und 34 der Neugeborenen (33,7\%) durchgeführt. Der Nachweis von Opiaten (außer Methadon) hatte dabei mit 34,5\% (beim Urinscreening der Mütter) bzw. 44,1\% (beim Urinscreening der Neugeborenen) den größten Anteil. An zweiter Stelle standen Cannabinoide (24,1\% bzw. 17,6\%), gefolgt von Benzodiazepinen (13,8\% bzw. 14,7\%), Kokain (10,3\% bzw. 8,8\%) und Barbituraten (3,4\% bzw. 5,9\%).

Die Kontrollgruppe gilt als drogenfreie Gruppe, ein übermäßiger Substanzkonsum (z.B. Alkohol, Nikotin, Schmerzmittel) kann aber nicht ausgeschlossen werden, wurde aber nur vereinzelt dokumentiert. 


\subsubsection{Substitution}

Genauere Angaben lassen sich in Bezug auf die Substitution machen: In der ZieglerExperimentalstichprobe fanden sich 56 Kinder (55,4\%), deren Mütter substituiert wurden; in der aktuellen Experimentalgruppe (EG) sind es entsprechend von insgesamt 57 Kindern 29 (50,9\%), deren Mütter während oder auch schon vor der Schwangerschaft eine Substitution erhielten. Das am häufigsten verwendete Substitutionsmittel war Methadon, nur in zwei Fällen wurde Remedacen verabreicht.

\subsubsection{HIV-Status}

In der Ziegler-Experimentalstichprobe waren 24 (23,8\%) der opiatabhängigen Mütter zum Zeitpunkt der Geburt HIV-positiv; die Kinder von 14 dieser Mütter sind in der aktuellen EG enthalten, der Anteil entspricht 24,6\%. Die Kontrollgruppe (KG) der aktuellen Stichprobe beinhaltet $9(42,9 \%)$ Kinder von zum Zeitpunkt der Geburt HIV-positiven Müttern, in der Ziegler-Kontrollgruppe waren 20 (40\%) der Mütter HIV-positiv. Sowohl für EG als auch für KG entsprechen die Prozentsätze der aktuellen der ursprünglichen Stichprobe.

\subsubsection{Neonatologische Daten der Kinder}

Tabelle 8.2 zeigt die Verteilung von Geburtstermin, Geburtsgewicht (kategorisiert) und Geschlecht der Experimentalgruppe und der Kontrollgruppe sowohl für die ursprüngliche als auch für die aktuelle Stichprobe.

Tab. 8.2 Geburtstermin, Geburtsgewicht und Geschlecht der Kinder

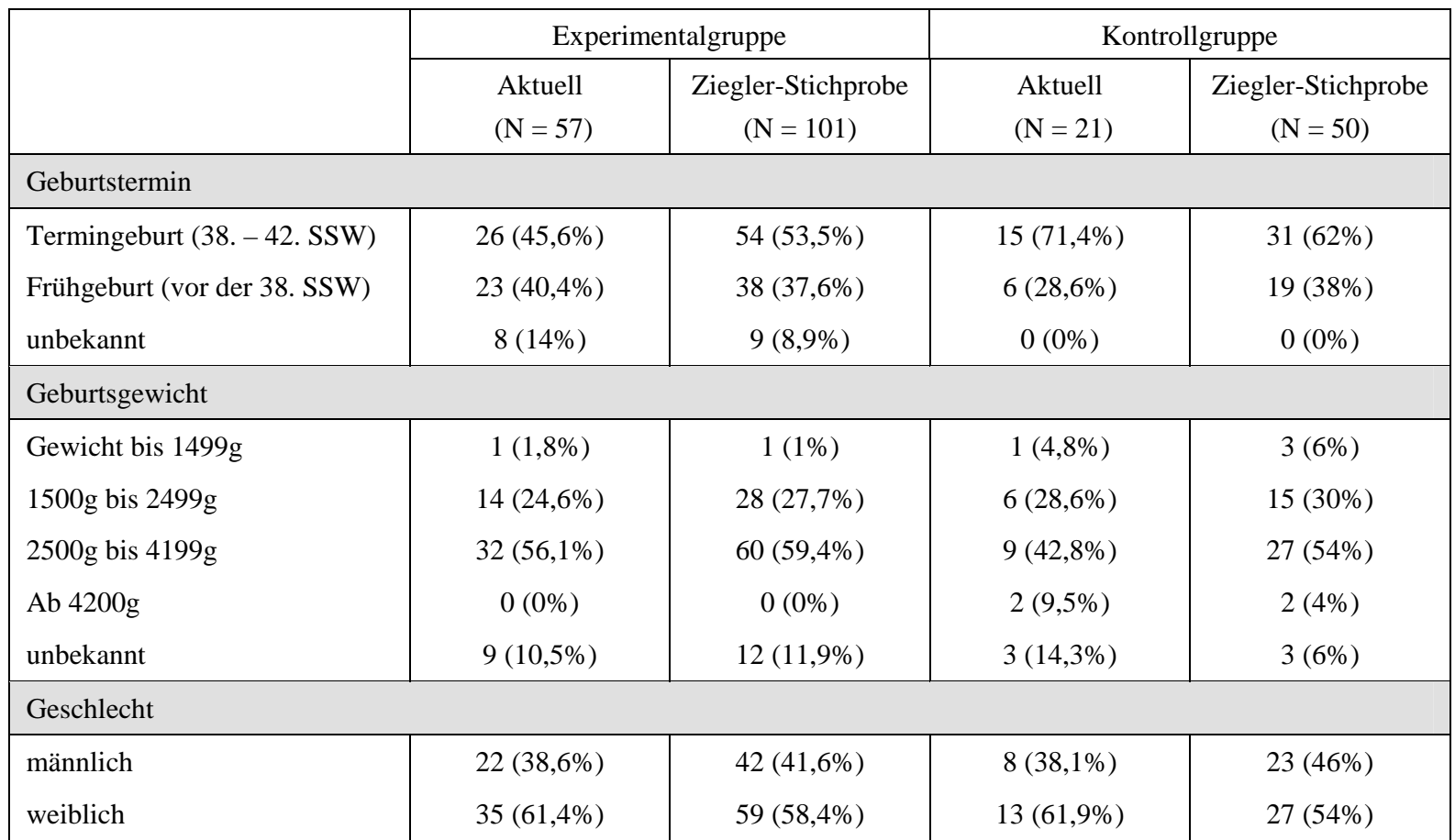


In der Experimentalgruppe der aktuellen Untersuchung (EG) ist der Anteil der Termin- und Frühgeburten annähernd gleich groß, während in der Kontrollgruppe die Termingeburten eindeutig überwiegen. In beiden Gruppen bilden die Kinder mit normalem Geburtsgewicht von $2500 \mathrm{~g}$ bis $4199 \mathrm{~g}$ die zahlenmäßig größte Gruppe; zwischen der aktuellen Stichprobe und der Ziegler-Stichprobe gab es hier nur wenige Verschiebungen. Der Anteil der Kinder mit niedrigem Geburtsgewicht beträgt durchgängig ca. ein Viertel.

Sowohl in der von Ziegler rekrutierten Experimental- und Kontrollgruppe als auch in den aktuellen Gruppen überwiegt der Anteil der Mädchen. Es fällt aber auf, dass sich die Quote sowohl in der EG als auch in der KG weiter zu Gunsten der Mädchen verschoben hat

\subsubsection{Medizinische Komplikationen der Kinder in der Kontrollgruppe}

Außer Frühgeburtlichkeit und niedrigem Geburtsgewicht waren das Vorliegen medizinischer Komplikationen eine Indikation für den Einschluss in die $\mathrm{KG}$ von Ziegler. In der ursprünglichen Stichprobe wiesen 7 Kinder eine kongenitale Anomalie auf, 14 Neugeborene litten an einer respiratorischen Anpassungsstörung, 9 Kinder mussten antibiotisch behandelt werden, 6 wiesen schwere gastrointestinale Störungen auf und in 3 Fällen wurden intrakranielle Blutungen festgestellt. In der aktuellen Stichprobe der Kontrollgruppe fanden sich von diesen Kindern vier mit einer kongenitalen Anomalie (Mikrozephalie, Pylorusstenose, Sichelfuß, Alkoholembryopathie), drei Kinder mit einer respiratorischen Anpassungsstörung, zwei Kinder mit einer antibiotisch zu behandelnden Infektion, zwei Kindern mit einer gastrointestinalen Störung und 1 Kind mit intrakraniellen Blutungen. In der ursprünglichen KG gab es 32 Nennungen medizinischer Komplikationen bei insgesamt 50 Kindern (64\%), das entspricht ungefähr dem Prozentsatz in der aktuellen KG von 57\% (12 Nennungen bei insgesamt 21 Kindern). Die Anzahl respiratorischer Anpassungsstörungen ist in der aktuellen Kontrollgruppe gegenüber der Ziegler-Kontrollgruppe unterrepräsentiert.

\subsubsection{Betreuungssituation}

\subsubsection{Betreuungsstatus ursprünglich und aktuell}

Die aktuelle Betreuungssituation in der EG hat sich gegenüber der ursprünglichen oft verändert. In Abbildung 8.1 ist der Betreuungsstatus nach der Entlassung aus dem Krankenhaus und zum aktuellen Zeitpunkt dargestellt. Die Entlassung aus dem Krankenhaus erfolgte in der EG auf Grund der Entzugssymptomatik und den damit verbundenen Komplikationen meist zwischen 6 und 8 Wochen, durchschnittlich nach 7 Wochen. In der KG 
war die Aufenthaltsdauer erheblich kürzer und die Kinder wurden im Durchschnitt nach 12 Tagen stationärer Behandlung entlassen.

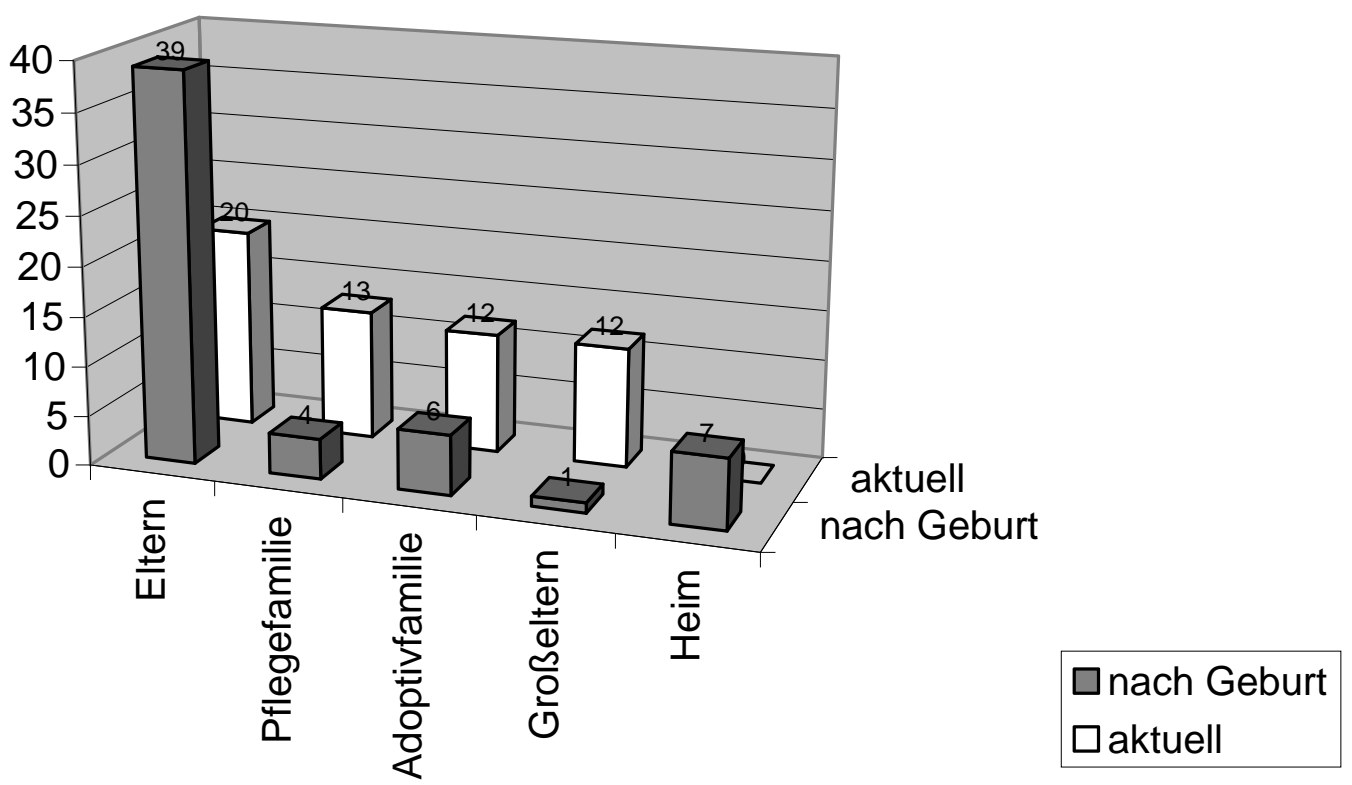

Abb. 8.1 Betreuungsverhältnisse in der EG $(\mathrm{N}=57)$

Von den 57 untersuchten Kindern in der EG wurden nach der Geburt ca. zwei Drittel (68\%) zu den leiblichen Eltern bzw. zur Mutter entlassen. In der Ausgangsstichprobe betrug dieser Anteil (71\%). Die restlichen Kinder wurden in Kinderheimen, Pflege- und Adoptivfamilien und in einem Fall bei den Großeltern untergebracht. 


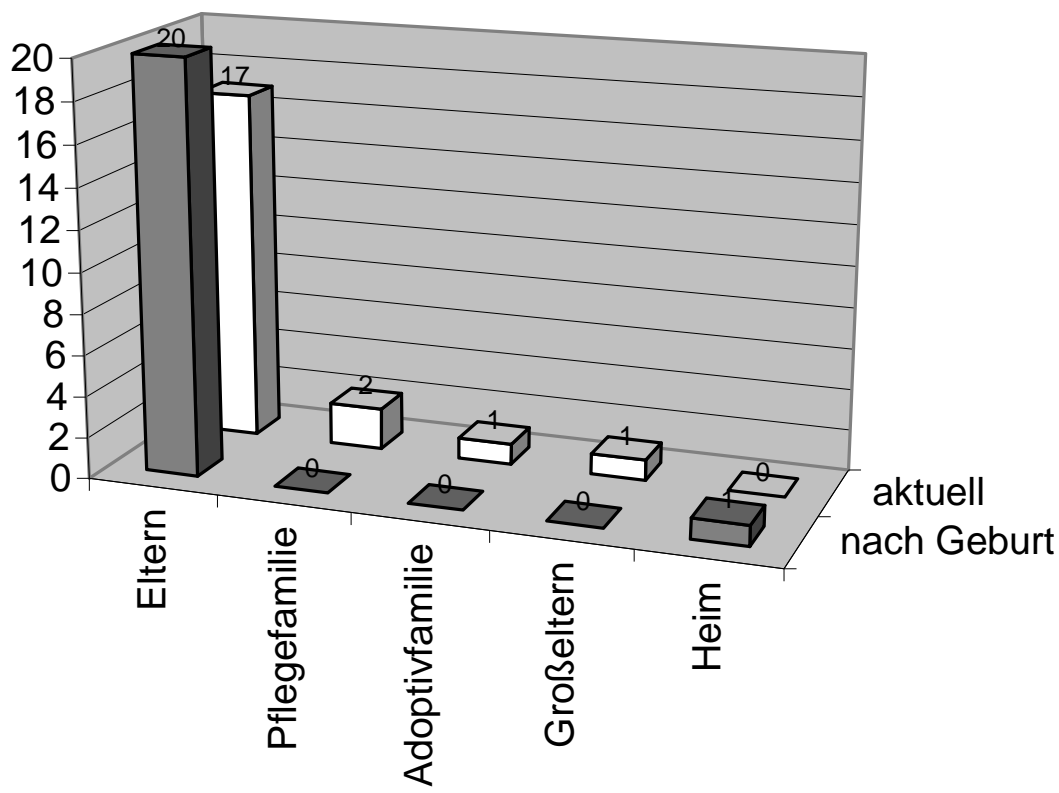

$\square$ nach Geburt $\square$ aktuell

Abb. 8.2 Betreuungsverhältnis in der $\mathrm{KG}(\mathrm{N}=21)$

Anders stellt sich die Situation in der KG dar (Abbildung 8.2). Mit einer Ausnahme konnten alle Kinder (95\%) nach der Geburt zu den Eltern entlassen werden. Entsprechend waren in der Kontrollgruppe von Ziegler 47 von 50 Kindern (94\%) nach Hause entlassen worden. Ein Kind wurde bereits gleich nach der Geburt gemeinsam mit seiner psychisch kranken Mutter zunächst im Mutter-Kind-Heim untergebracht, nach wenigen Monaten kam das Mädchen zu seinen heutigen Adoptiveltern. Die übrigen Betreuungswechsel erfolgten von den Eltern zu den Großeltern und in zwei Fällen zu Pflegeeltern.

Der Anteil der Kinder in der aktuellen Untersuchung, der ursprünglich zu ihren Eltern/Müttern nach Hause entlassen wurde, entspricht sowohl in der EG als auch in der KG dem Anteil der nach Hause entlassenen Kinder in der Ausgangsstichprobe. 


\subsubsection{Betreuungswechsel}

Nach der Gegenüberstellung der ursprünglichen (nach Entlassung aus der Klinik) und heutigen Wohnverhältnisse zeigt Tabelle 8.3 detaillierter, wohin die Betreuungswechsel (BW) erfolgten, bzw. wo die Kinder heute leben.

Tab. 8.3 Betreuungswechsel

\begin{tabular}{|c|c|c|}
\hline Entlassung/aktuelle Verhältnisse & EG & KG \\
\hline Nach Geburt entlassen zu beiden Elternteilen & $31(54 \%)$ & $17(81 \%)$ \\
\hline \multicolumn{3}{|l|}{ Davon leben aktuell ..... } \\
\hline bei beiden leiblichen Eltern & 9 & 9 \\
\hline Bei der Mutter alleine & 3 & 4 \\
\hline Bei den Großeltern/Großmutter & 7 & - \\
\hline Vater und Großmutter & 1 & 2 \\
\hline Vater und neue Partnerin & 2 & - \\
\hline Mutter und neuer Partner & 1 & \\
\hline Bei Pflegeeltern & 8 & 2 \\
\hline Nach Geburt entlassen zur Mutter & $8(14 \%)$ & $3(14 \%)$ \\
\hline \multicolumn{3}{|l|}{ Davon leben aktuell... } \\
\hline Bei der Mutter alleine & 1 & 2 \\
\hline Bei Mutter und neuem Partner & 3 & - \\
\hline Bei den Großeltern/Großmutter & 3 & 1 \\
\hline Bei Adoptiveltern & 1 & - \\
\hline Nach Geburt entlassen ins Kinderheim oder Mutter-Kind-Heim & $7(12 \%)$ & $1(5 \%)$ \\
\hline \multicolumn{3}{|l|}{ Davon leben aktuell... } \\
\hline Bei Pflegeeltern & 3 & 1 \\
\hline Bei Adoptiveltern & 3 & \\
\hline Bei Großmutter und neuem Partner & 1 & \\
\hline Nach Geburt entlassen zu Adoptiveltern & $6(11 \%)$ & \\
\hline \multicolumn{3}{|l|}{ Davon leben aktuell.... } \\
\hline Bei Adoptiveltern & 6 & \\
\hline Nach Geburt entlassen zu Pflegeeltern & $4(7 \%)$ & \\
\hline \multicolumn{3}{|l|}{ Davon leben aktuell.... } \\
\hline Bei Pflegeeltern & 2 & \\
\hline Bei Adoptiveltern & 2 & \\
\hline Nach Geburt entlassen zu Großmutter & $1(2 \%)$ & \\
\hline Lebt aktuell bei Großmutter & 1 & \\
\hline Insgesamt & $57(100 \%)$ & $21(100 \%)$ \\
\hline
\end{tabular}

Die Entlassung zu Adoptiv- oder Pflegeeltern stellt das stabilste Betreuungsverhältnis nach der Geburt dar. Von den zu beiden leiblichen Eltern entlassenen Kindern lebt nur noch ein 
knappes Drittel nach wie vor bei beiden Eltern, der größte Teil dieser Kinder lebt heute bei einem Großelternteil bzw. bei Pflege- oder Adoptiveltern. In nur einem Fall wurde ein Kind direkt nach der Geburt zur Großmutter entlassen.

\section{Gründe für späte Betreuungswechsel}

In der Arbeit von Ziegler wurde deutlich, dass Mütter von nach der Geburt fremdplatzierten Kindern sich von Müttern nicht-fremdplatzierter Kinder deutlich unterscheiden. Erstere gehörten einer niedrigeren sozialen Schicht an, wiesen eine höhere Arbeitslosigkeit und eine deutlich schlechtere Wohnsituation auf. Sie lebten zu einem größeren Prozentsatz ohne festen Partner. Das Fürsorgeverhalten von Müttern fremdplatzierter Kinder war in $75 \%$ der Fälle mangelhaft bzw. kümmerten sich die Mütter überhaupt nicht um ihr Kind, während das Fürsorgeverhalten bei den Müttern nicht-fremdplatzierter Kinder größtenteils gut bis sehr gut war.

Da ein großer Teil der zunächst zu den leiblichen Müttern entlassenen Kinder in der Folge doch fremdplatziert wird, stellt sich die Frage nach den Ursachen für diese erst nach einigen Monaten bis Jahren erfolgten Trennungen. In unserer Stichprobe waren die in Tabelle 8.4 angeführten Umstände und Gründe ausschlaggebend:

Tab. 8.4 Gründe für spätere Fremdplatzierungen

\begin{tabular}{|l|c|}
\hline Umstände bzw. Gründe für spätere Fremdplatzierung & \\
\hline Krankheit der Mutter & 4 \\
\hline Tod der Mutter & 3 \\
\hline Mutter ließ Kind bei Großeltern & 6 \\
\hline Massive Vernachlässigung & 3 \\
\hline Inhaftierung der Mutter & 1 \\
\hline unklar & 3 \\
\hline
\end{tabular}

In vier Fällen war die starke Beeinträchtigung der Mutter durch Krankheit (HIV und Folgeerkrankungen, Hepatitis C) die Hauptursache für die Fremdunterbringung. Drei der Mütter starben (an AIDS bzw. an einer Überdosis) als die Kinder zwischen 1 und 4 Jahren alt waren. 6 der Mütter, deren Kinder ursprünglich zu ihnen nach Hause entlassen worden waren, ließen ihre Kinder bei den Großeltern. Dies geschah manchmal plötzlich und ohne Vorankündigung, manchmal auch schrittweise, in dem die Mütter ihre Kinder immer mehr der Großmutter überließen, bzw. selbst immer weniger nach Hause kamen. Eindeutige Gründe ließen sich rückblickend und aus den Erzählungen der aktuellen 
Hauptbezugspersonen nicht mehr genau rekonstruieren, in allen Fällen spielte aber anhaltender Drogenkonsum und/oder massive Paarkonflikte eine Rolle. Bei 3 der Kinder war Vernachlässigung der Hauptgrund für die Trennung von der Mutter. In zwei dieser Fälle erfolgte eine Anzeige, eine Mutter hinterließ ihre Kinder selbst bei der Sozialstation.

\subsubsection{Gruppeneinteilung nach dem Betreuungsstatus (EG1, EG2, EG3, KG1)}

Wie in Kapitel 7 bei der Formulierung der Hypothesen beschrieben wurde innerhalb der Experimentalgruppe eine Untergruppierung auf der Basis der unterschiedlichen Betreuungsverhältnisse vorgenommen. Die Zuordnung zu den Gruppen richtete sich nach Anzahl und Zeitpunkt der Betreuungswechsel. Bei den Kindern und Jugendlichen, bei denen nur eine Rückmeldung per Fragebogen zur Verfügung stand, konnte aufgrund fehlender Informationen keine Gruppenzuteilung vorgenommen werden. Insgesamt konnten 51 Kinder der EG und 17 Kinder der KG den Gruppen zugeteilt werden.

- EG1 (Eltern): alle Kinder mit pränataler Opiatexposition, die kontinuierlich bei mindestens einem leiblichen Elternteil lebten und aktuell leben.

- EG2 (Fremdbetreuung zu einem frühen Zeitpunkt): alle Kinder mit pränataler Opiatexposition, die bei Pflege- oder Adoptiveltern bzw. bei den Großeltern aufwachsen und bei denen der Wechsel zu den Ersatzeltern meist in den ersten Monaten erfolgte. Die Aufnahme in die neue Familie erfolgte dabei entweder direkt nach der Entlassung aus der Klinik oder nach meist wenigen Monaten bei den Eltern/der Mutter.

- EG3 (Fremdbetreuung zu einem späten Zeitpunkt): alle Kinder mit pränataler Opiatexposition, die bei Pflege-, Adoptiv- oder den Großeltern aufwachsen, deren Aufnahme bei den Ersatzeltern aber erst nach dem ersten Lebensjahr erfolgte und nach meist mehreren vorangegangenen Bezugspersonenwechseln.

- KG1 (Kontrollgruppe Eltern): alle Kinder ohne pränatale Opiatexposition (Kontrollgruppe), die bei mindestens einem leiblichen Elternteil aufwachsen. Um Vergleichbarkeit mit den anderen drei Gruppen zu gewährleisten, wurden innerhalb dieser Gruppe die vier Kinder, die durch Großeltern bzw. Pflegeeltern betreut wurden herausgenommen. Alle verbleibenden Kinder der KG1 sind somit von mindestens einem Elternteil kontinuierlich betreut worden.

In Tabelle 8.5 ist dargestellt, wer die bisherige Hauptbezugsperson bzw. hauptbetreuende Person war. In EG1 und KG1 sind dies definitionsgemäß die leiblichen Eltern bzw. die 
Mütter. In der EG2, der Gruppe mit frühem Betreuungswechsel wird jeweils die Hälfte der Kinder von Pflege/Adoptiveltern bzw. den Großmüttern betreut. Die Zuteilung einer „bisher hauptbetreuenden Person“ gestaltet sich in der EG3 am schwierigsten, da die erlebten Betreuungswechsel wie beschrieben wiederholt und oft erst $\mathrm{zu}$ einem späten Zeitpunkt stattfanden. Bei 3 Kindern der EG3 wurde die Mutter als „bisher hauptbetreuende Person“ definiert: Eines dieser Kinder, ein Mädchen, erlebte bisher 5 Betreuungswechsel, die meiste Zeit ihre Lebens verbrachte sie aber bei ihrer Mutter. Die beiden anderen Kinder waren zum Zeitpunkt der Untersuchung 8 und 10 Jahre alt, der Wechsel zur Pflegefamilie bzw. zur Großmutter erfolgte aber erst mit 5 bzw. 8 Jahren.

Tab. 8.5 Hauptbezugspersonen

\begin{tabular}{|l|c|c|c|c|}
\hline Bisher hauptbetreuende Bezugsperson & EG1 $(\mathrm{N}=18)$ & EG2 $(\mathrm{N}=19)$ & EG3 $(\mathrm{N}=14)$ & KG1 $(\mathrm{N}=17)$ \\
\hline Leibliche Eltern/Mutter/Vater & 18 & & 3 & 17 \\
\hline Pflege-/Adoptiveltern & & 9 & 10 & \\
\hline Großeltern & & 10 & 1 & \\
\hline
\end{tabular}

\section{$\underline{\text { Anzahl der Betreuungswechsel }}$}

Die Anzahl der Betreuungswechsel ist definitionsgemäß in der Gruppe EG3 am höchsten. Tabelle 8.6 zeigt die genaue Aufteilung der Betreuungswechsel für jede Gruppe. Wurden die Kinder in einem Alter von wenigen Wochen nach dem Krankenhausaufenthalt direkt zu den Pflege-/Adoptiveltern entlassen, so wurde dies nicht als Betreuungswechsel gezählt. Sobald eine Zwischenstation, z.B. ein Aufenthalt in einem Kinderheim dazukam, galt dies als Betreuungswechsel. Zusätzlich wird die durchschnittliche Anzahl der Betreuungswechsel angegeben $($ Mittelwert $=$ MW, Standardabweichung $=$ SD $)$.

Tab. 8.6 Anzahl der Betreuungswechsel

\begin{tabular}{|l|c|c|c|c|}
\hline Anzahl Betreuungswechsel & EG1 $(\mathrm{N}=18)$ & EG2 $(\mathrm{N}=19)$ & EG3 $(\mathrm{N}=14)$ & KG1 $(\mathrm{N}=17)$ \\
\hline Kein Wechsel & 18 & 7 & & 16 \\
\hline 1 Wechsel & & 10 & 3 & 1 \\
\hline 2 Wechsel & & 2 & 4 & \\
\hline 3 Wechsel & & & 3 & \\
\hline 4 Wechsel & & & 1 & \\
\hline 5 Wechsel & & & & \\
\hline Durchschnittliche Anzahl & & & & \\
\hline MW (SD) & 0 & $0,74(0,65)$ & $2,64(1,28)$ & $0,058(0,24)$ \\
\hline
\end{tabular}

In der EG2 beträgt das durchschnittliche Alter beim ersten, und meistens auch einzigen Betreuungswechsel 7,4 Monate, wobei in 13 von 19 Fällen der Wechsel vor bzw. mit einem Alter von 6 Monaten erfolgte. In der EG3 beträgt das durchschnittliche Alter beim ersten von 
meist mehreren Wechseln 24,2 Monate, der früheste Wechsel fand mit einem Jahr statt, der späteste mit 8 Jahren.

\section{Anzahl der Betreuungswechsel und Hauptbezugsperson}

Tabelle 8.7 zeigt die Anzahl der Betreuungswechsel in Abhängigkeit des aktuellen Betreuungsverhältnisses.

Tab. 8.7 Betreuungswechsel und Betreuungsverhältnis

\begin{tabular}{|l|c|c|c|c|}
\hline Anzahl Betreuungswechsel & $\begin{array}{c}\text { Leibliche Eltern } \\
(\mathbf{N}=\mathbf{3 7})\end{array}$ & $\begin{array}{c}\text { Pflegeeltern } \\
(\mathbf{N = 1 1})\end{array}$ & $\begin{array}{c}\text { Adoptiveltern } \\
(\mathbf{N}=\mathbf{9})\end{array}$ & $\begin{array}{c}\text { Großeltern } \\
(\mathbf{N}=\mathbf{1 2})\end{array}$ \\
\hline Kein Wechsel & 33 & 1 & 6 & 1 \\
\hline 1 Wechsel & 2 & 3 & 2 & 2 \\
\hline 2 Wechsel & & 3 & 1 & \\
\hline 3 Wechsel & 1 & 2 & & \\
\hline 4 Wechsel & 1 & 2 & & \\
\hline 5 Wechsel & & & & $1,08(0,51)$ \\
\hline Durchschnittliche Anzahl & $0,27(0,96)$ & $2,09(1,30)$ & $0,89(1,36)$ & \\
\hline MW (SD) &
\end{tabular}

Kinder, die bei Pflegefamilien aufwachsen, haben auch die größte Anzahl an Betreuungswechseln erlebt. Kinder die von den Großeltern betreut werden, erleben in der Mehrzahl der Fälle einen Wechsel - die Großeltern übernehmen die Verantwortung von den Eltern bzw. der Mutter.

Eine genaue Auflistung der Betreuungsverläufe in den vier Gruppen mit Anzahl und Zeitpunkt der Wechsel und jeweiliger Hauptbezugsperson befindet sich im Anhang.

\subsubsection{EG1, EG2, EG3, KG1 - Charakteristika der leiblichen Mütter}

In den nachfolgenden Tabellen sind Charakteristika der leiblichen Mütter aufgelistet. Sämtliche Daten zu diesen Auflistungen stammen aus der Dokumentation von Ziegler mit dem Datenerfassungsbogen (KDAM-Bogen), der auf S 59 genauer beschrieben wird. Alter der Mutter bei Entbindung, Parität und sozioökonomischer Status gehörten zu den ursprünglichen Parallelisierungskriterien; weiterhin sind die Nationalität der Mütter, Substitutionsstatus zum Zeitpunkt der Geburt sowie Zuwendungsverhalten und Kooperation auf der Station angeführt. 


\subsubsection{Alter bei Entbindung}

Tabelle 8.8 zeigt das durchschnittliche Alter der leiblichen Mütter zum Zeitpunkt der Entbindung.

Tab. 8.8 Alter der Mutter bei der Entbindung

\begin{tabular}{|l|c|c|c|c|}
\hline Alter & EG1 $(\mathrm{N}=18)$ & EG2 $(\mathrm{N}=19)$ & EG3 $(\mathrm{N}=14)$ & KG1 $(\mathrm{N}=17)$ \\
\hline MW $(\mathrm{SD})$ & $27,6(4,6)$ & $25,3(6,6)$ & $25,7(5,3)$ & $28(4,6)$ \\
\hline
\end{tabular}

Das Alter der Mutter ist in allen vier Gruppen annähernd gleich: $F(3,60)=1,010 ; p=0,395$.

\subsubsection{Parität}

Die Anzahl der ausgetragenen Schwangerschaften war in der ursprünglichen Stichprobe parallelisiert. In Tabelle 8.9 ist die Anzahl der ausgetragenen Schwangerschaften der leiblichen Mütter angeführt.

Tab. 8.9 Anzahl der ausgetragenen Schwangerschaften

\begin{tabular}{|l|c|c|c|c|}
\hline Ausgetragene & EG1 $(\mathrm{N}=18)$ & EG2 $(\mathrm{N}=19)$ & EG3 $(\mathrm{N}=14)$ & KG1 $(\mathrm{N}=17)$ \\
\hline 1 & 7 & 13 & 8 & 7 \\
\hline 2 & 5 & 1 & 4 & 8 \\
\hline 3 & 3 & 2 & 1 & 2 \\
\hline 4 & 1 & 1 & 1 & - \\
\hline Unbekannt & 2 & 2 & - & - \\
\hline Durchschnittliche Anzahl & & & & $1,71(0,69)$ \\
\hline MW (SD) & $1,88(0,96)$ & $1,47(0,94)$ & $1,64(0,93)$ & \\
\hline
\end{tabular}

Die Anzahl der ausgetragenen Schwangerschaften unterscheidet sich in den vier Gruppen nicht signifikant voneinander: $F(3,60)=0,591 ; p=0,623$.

\subsubsection{Sozio-ökonomischer Status}

Die Mütter der ursprünglichen Stichprobe gehörten, wie aus Tabelle 8.10 ersichtlich, fast vollständig der Unterschicht an.

Tab. 8.10 Schichtzugehörigkeit der leiblichen Mütter

\begin{tabular}{|l|c|c|c|c|}
\hline Soziale Schicht & EG1 $(\mathrm{N}=18)$ & EG2 $(\mathrm{N}=19)$ & EG3 $(\mathrm{N}=14)$ & KG1 (N=17) \\
\hline Untere Unterschicht (1) & - & $4(21,1 \%)$ & - & - \\
\hline Mittlere Unterschicht (2) & $12(66,7 \%)$ & $12(63,2 \%)$ & $10(71,4 \%)$ & $7(41,2 \%)$ \\
\hline Obere Unterschicht (3) & $3(16,7 \%)$ & $2(10,5 \%)$ & $4(28,6 \%)$ & $6(35,3 \%)$ \\
\hline Untere Mittelschicht (4) & $2(11,1 \%)$ & - & - & $4(23,5 \%)$ \\
\hline Unbekannt & $1(5,5 \%)$ & $1(5,2 \%)$ & - & - \\
\hline Durchschnittliche soz. Schicht & & & & $2,82(0,81)$ \\
\hline MW (SD) & $2,41(0,71)$ & $1,89(0,58)$ & $2,29(0,47)$ & \\
\hline
\end{tabular}


Bildet man den Mittelwert aus der Schichtzugehörigkeit, zeigt sich, dass die Mütter der Kinder der KG1 zum Zeitpunkt der Geburt den durchschnittlich höchsten SES aufweisen, und zwar signifikant höher als die leiblichen Mütter der EG2, bei denen der SES zum Zeitpunkt der Geburt am niedrigsten war: F $(3,62)=5,89 ; p=0,001$

\subsubsection{Substitution bei Geburt}

Da die Substitution einen Zusammenhang mit der Rate der Fremdplatzierungen aufweist, gibt es, wie aus Tabelle 8.11 erkennbar, erwartungsgemäß große diesbezügliche Unterschiede in den Betreuungsgruppen.

Tab. 8.11 Substitutionsstatus zum Zeitpunkt der Geburt

\begin{tabular}{|l|c|c|c|}
\hline Substitutionsstatus & EG1 $(\mathbf{N}=\mathbf{1 8})$ & EG2 $(\mathbf{N}=\mathbf{1 9})$ & EG3 (N = 14) \\
\hline Substitution & $13(72,2 \%)$ & $4(21,1 \%)$ & $9(64,3 \%)$ \\
\hline Keine Substitution & $4(22,2 \%)$ & $14(73,7 \%)$ & $5(35,7 \%)$ \\
\hline Unbekannt & $1(5,6 \%)$ & $1(5,2 \%)$ & - \\
\hline
\end{tabular}

In der EG1, der Gruppe der bei den leiblichen Eltern verbliebenen Kinder, war die Mehrheit der Mütter zum Zeitpunkt der Geburt substituiert, in der EG2 ist das Verhältnis genau umgekehrt. Es besteht keine Gleichverteilung innerhalb der Gruppen (Pearson Chi-Quadrat $26,69, \mathrm{df}=3, \mathrm{p}<0,001)$.

\subsubsection{Nationalität der Mütter}

Die Nationalitäten der Mütter waren überwiegend deutsch, wobei der Anteil der deutschen Mütter in der KG doch deutlich kleiner ist als in der EG. Insgesamt entsprechen die Raten der deutschen Mütter in der aktuellen Stichprobe auch dem Anteil der deutschen Mütter in der Ursprungsstichprobe von Ziegler (88\% in der EG, 64\% in der $\mathrm{KG}$ ).

Tab. 8.12 Nationalität der leiblichen Mütter

\begin{tabular}{|l|c|c|c|c|}
\hline Nationalität & EG1 $(\mathrm{N}=18)$ & EG2 $(\mathrm{N}=19)$ & EG3 $(\mathrm{N}=14)$ & KG1 $(\mathrm{N}=17)$ \\
\hline Deutschland & $15(83,3 \%)$ & $14(73,7 \%)$ & $14(100 \%)$ & $11(64,7 \%)$ \\
\hline Türkei & & & & $2(11,7 \%)$ \\
\hline Italien & $1(5,6 \%)$ & & & $1(5,9 \%)$ \\
\hline Spanien & & & & $1(5,9 \%)$ \\
\hline Ex-Jugoslawien & & $2(10,5 \%)$ & & $2(11,7 \%)$ \\
\hline Kolumbien & & & & \\
\hline Afrika & & & & \\
\hline Unbekannt & $2(11,1 \%)$ & $3(15,8 \%)$ & & \\
\hline
\end{tabular}




\subsubsection{Zuwendungsverhalten und Kooperation zum Zeitpunkt der Geburt}

Vom Krankenhauspersonal wurde das beobachtete Zuwendungs- und Besuchsverhalten der Mütter auf der Station dokumentiert.

Tab. 8.13 Zuwendungsverhalten und Besuche auf Station

\begin{tabular}{|c|c|c|c|c|}
\hline Zuwendungsverhalten & EG1 $(N=18)$ & EG2 $(N=19)$ & EG3 $(N=14)$ & KG1 $(\mathrm{N}=17)$ \\
\hline Unauffällig & 15 & 8 & 10 & 16 \\
\hline Auffällig & 1 & 3 & 4 & 1 \\
\hline Unbekannt/nicht beurteilbar & 2 & 8 & - & - \\
\hline \multicolumn{5}{|c|}{ Besuch der Eltern auf der Station } \\
\hline Regelmäßig/zuverlässig (1) & 15 & 5 & 8 & 17 \\
\hline $\mathrm{Ab}$ und zu/unregelmäßig (2) & 2 & 2 & 4 & \\
\hline Sehr selten (3) & & 5 & 1 & \\
\hline Überhaupt nicht (4) & & 5 & & \\
\hline Unbekannt/nicht beurteilbar & 1 & 2 & 1 & - \\
\hline \multicolumn{5}{|l|}{ Durchschnittlicher Besuch } \\
\hline MW (SD) & $1,12(0,33)$ & $2,59(1,23)$ & $1,46(0,66)$ & $1(0)$ \\
\hline \multicolumn{5}{|c|}{ Kooperationsbereitschaft der Eltern } \\
\hline Gut (1) & 11 & 3 & 7 & 16 \\
\hline Mäßig gut (2) & 5 & 6 & 2 & 1 \\
\hline Schwach (3) & - & 2 & 3 & - \\
\hline Schlecht (4) & 1 & 1 & 2 & - \\
\hline Unbekannt/nicht beurteilbar & 1 & 7 & - & - \\
\hline \multicolumn{5}{|c|}{ Durchschnittliche Kooperation } \\
\hline MW (SD) & $1,47(0,8)$ & $2,08(0,90)$ & $2(1,18)$ & $1,06(0,24)$ \\
\hline
\end{tabular}

Im Zuwendungsverhalten zeigen sich zwischen den einzelnen Gruppen keine signifikanten Verteilungsunterschiede (Pearson Chi-Quadrat 5,231, df =3, p =0,156). Zur Überprüfung möglicher Unterschiede im Besuchsverhalten wurde der Mittelwert berechnet - je geringer der Wert, desto unauffälliger war das Besuchsverhalten. In der Varianzanalyse zeigte sich, dass die Mütter der Gruppe EG2 ein signifikant auffälligeres Verhalten aufwiesen als die Mütter der drei anderen Gruppen: F $(3,60)=17,193 ; p<0,001)$. Die $p$-Werte der paarweisen Mittelwertsvergleiche (EG2 vs. EG1, EG3, KG1) mittels Scheffé-Prozedur betrugen $\mathrm{p}<=$ 0.001. In der Kooperationsbereitschaft der Eltern zeigten sich ebenfalls signifikante Unterschiede $(\mathrm{F}(3,56)=5,051 ; \mathrm{p}=0,004)-$ die durchschnittliche Kooperation der Mütter/Eltern der EG2 ( $\mathrm{p}=0,018)$ und EG3 $(\mathrm{p}=0,026)$ war signifikant geringer als der KG1. Auch hier sind die Ergebnisse wenig überraschend, denn von den leiblichen Eltern der EG2, die das Sorgerecht verloren haben, wurde durchaus auch ein auffälligeres Besuchsverhalten sowie eine geringere Kooperation erwartet. 


\subsubsection{EG1, EG2, EG3, KG1 - Charakteristika der aktuellen Bezugspersonen}

In den nachfolgenden Tabellen sind Variablen der aktuellen Bezugspersonen aufgelistet. Die Daten für diese Auflistungen stammen aus den Erhebungen der aktuellen Untersuchung. Die Darstellung dient nicht der Überprüfung der Vergleichbarkeit über die vier Gruppen, sondern es soll damit die unterschiedliche Situation bezüglich der psychosozialen Risiken in den vier Gruppen veranschaulicht werden. Teilweise deutliche Unterschiede in diesen Variablen entsprechen dabei durchaus den Erwartungen.

\subsubsection{Schulbildung, Lebensunterhalt und Beziehungsstatus}

Die psychosozialen Charakteristika der Mütter bzw. der betreuenden Personen sind in den nachfolgenden Tabellen veranschaulicht.

Tab. 8.14 Schulbildung

\begin{tabular}{|l|c|c|c|c|}
\hline & EG1 $(\mathbf{N}=\mathbf{1 8})$ & EG2 $(\mathbf{N}=19)$ & EG3 (N= 14) & KG1 (N= 14) \\
\hline Ohne Abschluss & - & - & $1(7,1 \%)$ & $3(17,6 \%)$ \\
\hline Hauptschule & $7(38,9 \%)$ & $6(31,6 \%)$ & $2(14,3 \%)$ & $3(17,6 \%)$ \\
\hline Mittlere Reife & $5(27,8 \%)$ & $4(21,1 \%)$ & $3(21,4 \%)$ & $6(35,3 \%)$ \\
\hline Abitur & $3(16,7 \%)$ & $1(5,3 \%)$ & $2(14,3 \%)$ & $1(5,9 \%)$ \\
\hline Berufsfachschule & $1(5,6 \%)$ & $5(26,3 \%)$ & $1(7,1 \%)$ & $1(5,9 \%)$ \\
\hline Hochschulabschluss & - & $2(10,5 \%)$ & $5(35,7 \%)$ & - \\
\hline Unbekannt & $2(11,1 \%)$ & $1(5,3 \%)$ & & $3(17,6 \%)$ \\
\hline
\end{tabular}

Wie Tabelle 8.14 zeigt, verfügen nur Pflege- und Adoptiveltern aus den Gruppen EG2 und EG3 über einen Hochschulabschluss; überrepräsentiert ist im Vergleich zu den anderen Gruppen auch der Hauptschulabschluss in der Gruppe EG1. Mütter ohne jeden Schulabschluss finden sich nur in den Gruppen EG3 und KG1.

\section{Lebensunterhalt}

Deutliche Unterschiede zeigen sich bezüglich der Hauptquelle des Lebensunterhalts (Tabelle 8.15): Die (ehemals) opiatabhängigen Mütter leben fast zur Hälfte von Sozialhilfe und in der Gruppe EG3 sind es ebenfalls die leiblichen Mütter, welche Sozialhilfeempfang als Lebensunterhalt definieren. Unterstützung durch Partner, Freunde spielt in allen Gruppen eine Rolle, darin ist auch die Hausfrauentätigkeit beinhaltet. Ersatzmütter und Großmütter gehen zu einem größeren Teil einer geregelten Arbeit nach als die leiblichen Mütter der EG und KG. 
Tab. 8.15 Hauptquelle des Lebensunterhalts

\begin{tabular}{|l|c|c|c|c|}
\hline & EG1 (N= 18) & EG2 (N= 19) & EG3 (N= 14) & KG1 (N=14) \\
\hline Erwerbstätigkeit & $4(22 \%)$ & $7(37 \%)$ & $5(36 \%)$ & $3(17.5 \%)$ \\
\hline Arbeitslosengeld bzw. -hilfe & - & - & $1(7 \%)$ & $2(12 \%)$ \\
\hline Sozialhilfe & $8(44 \%)$ & - & $2(14 \%)$ & $2(12 \%)$ \\
\hline Rente & $1(6 \%)$ & $6(32 \%)$ & - & $2(12 \%)$ \\
\hline Unterstützung durch Partner und Freunde & $4(22 \%)$ & $5(26 \%)$ & $6(43 \%)$ & $5(29 \%)$ \\
\hline Andere Einkünfte & - & - & - & $1(5.5 \%)$ \\
\hline Unbekannt & $1(6 \%)$ & $1(5 \%)$ & - & $2(12 \%)$ \\
\hline
\end{tabular}

\section{Beziehungsstatus}

Tabelle 8.16 zeigt die aktuelle Partnerbeziehung der Bezugspersonen. Während in der EG1 80\% der leiblichen Mütter in einer festen Partnerschaft leben sind es bei den leiblichen Eltern der KG1 nur 60\%. In der EG2 und EG3 sind es ausschließlich die Großmütter, die als Beziehungsstatus „alleinstehend“ angeben, alle Adoptiv- und Pflegemütter hingegen leben in einer festen Partnerschaft.

Tab. 8.16 Beziehungsstatus der Mütter und Ersatzmütter

\begin{tabular}{|l|c|c|c|c|}
\hline & EG1 (N = 18) & EG2 $(\mathbf{N}=\mathbf{1 9})$ & EG3 $(\mathbf{N}=\mathbf{1 4})$ & KG1 (N= 14) \\
\hline Alleinstehend & $2(11,1 \%)$ & $2(11 \%)$ & $3(21 \%)$ & $5(29,4 \%)$ \\
\hline Feste Beziehung & $14(77,8 \%)$ & $16(84 \%)$ & $11(79 \%)$ & $9(53 \%)$ \\
\hline Unbekannt & $2(11,1 \%)$ & $1(5 \%)$ & - & $3(17,6 \%)$ \\
\hline
\end{tabular}

\subsubsection{Aktuelle körperliche Verfassung der Bezugspersonen}

Die körperliche Verfassung der Mütter bzw. Ersatzmütter in Bezug auf HIV und Hepatitis C ist in Tabelle 8.17 aufgeführt. Eine der leiblichen Mütter der EG1 leidet an Hepatitis $\mathrm{C}$ und ist zugleich HIV-positiv. Dasselbe gilt für die zwei leiblichen Mütter der EG3, die als Hauptbezugsperson gelten - beide leiden unter beiden Erkrankungen. In der Kontrollgruppe sind 6 Mütter HIV-positiv. Der Unterschied zur in Punkt 8.2.1.3 angegebenen Zahl von 9 HIV-positiven Müttern in der KG kommt dadurch zustande, dass zwei der Kinder bei ihrem Vater leben und ein Kind aufgrund eines Fremdbetreuungsverhältnisses nicht in der KG1 inkludiert ist. In der Gruppe der Adoptiv- und Pflegemütter findet sich kein Fall von Hepatitis C oder HIV. 
Tab. 8.17 Hep. C und HIV-Infektion der Bezugsperson

\begin{tabular}{|l|c|c|c|c|}
\hline & EG1 $(\mathbf{N}=\mathbf{1 8})$ & EG2 $(\mathbf{N}=\mathbf{1 9})$ & EG3 $(\mathbf{N}=\mathbf{1 4})$ & KG1 (N = 17) \\
\hline Hepatitis C & 13 & 0 & 2 & 0 \\
\hline HIV-Infektion & 1 & 0 & 2 & 6 \\
\hline Unbekannt & 1 & - & - & - \\
\hline
\end{tabular}

\subsubsection{Aktueller Alkohol- und Drogenkonsum}

Die Angaben zum aktuellen Alkohol- und Drogenkonsum stützen sich auf die Exploration mittels des Addiction Severity Index (ASI) mit der jeweils hauptbetreuenden Bezugsperson. Der ASI wurde zu Beginn der Erhebung aufgrund eines ursprünglich anders geplanten Vorgehens teilweise mit der Bezugsperson (z.B. mit der Großmutter) über die leibliche Mutter durchgeführt. In diesen Fällen fehlen die Angaben zur Hauptbezugsperson, weshalb die Zahl der Fälle in diesen Gruppen kleiner ist. Der Alkoholkonsum ist definiert als Alkoholkonsum oberhalb der Gefährdungsgrenze, d.h. 5 oder mehr alkoholische Trinkeinheiten pro Tag an mindestens drei Tagen in der Woche oder der Konsum von Alkohol bis zum Rauschzustand an mindestens 2 aufeinanderfolgenden Tagen in der Woche. Eine der leiblichen Mütter konsumiert nach wie vor fast täglich Heroin, fast die Hälfte ist weiterhin methadonsubstituiert. Cannabis wird von 2 Müttern in der EG1 konsumiert, wobei die Häufigkeit mit einmal und fünfzehnmal innerhalb von 30 Tagen angegeben wird. Auch in der Kontrollgruppe gibt eine Mutter an einmal innerhalb eines Monats Cannabis zu konsumieren. In der EG3 stammen die Angaben zum Cannabiskonsum von den beiden leiblichen Müttern, bei denen die Kinder auch den überwiegenden Teil ihres bisherigen Lebens verbracht haben. Alle anderen Bezugspersonen in der EG3 verneinen Alkohol- und Drogenkonsum.

Tab. 8.18 Alkohol- und Drogenkonsum der Bezugspersonen

\begin{tabular}{|l|c|c|c|c|}
\hline & EG1 $(\mathbf{N}=\mathbf{1 6})$ & EG2 $(\mathbf{N}=\mathbf{1 1})$ & EG3 (N = 14) & KG1 (N = 12) \\
\hline Alkohol & $2(12,5 \%)$ & 0 & 0 & 0 \\
\hline Heroin & $1(6,25 \%)$ & 0 & 0 & 0 \\
\hline Methadon & $7(43,75 \%)$ & 0 & 0 & 0 \\
\hline Cannabis & $2(12,5 \%)$ & 0 & $2(14,28 \%)$ & $1(8,33 \%)$ \\
\hline
\end{tabular}




\subsubsection{EG1, EG2, EG3, KG1 - Charakteristika der Kinder}

In den nachfolgenden Tabellen sind demographische und gesundheitliche Charakteristika der Kinder aus der aktuellen Erhebung aufgelistet.

\subsubsection{Geschlecht, Altersverteilung und Schulbesuch}

Tabelle 8.19 zeigt die Verteilung von Alter und Geschlecht der Kinder in den vier Subgruppen.

Tab. 8.19 Alter und Geschlecht der Kinder

\begin{tabular}{|l|c|c|c|c|c|}
\hline Geschlecht & EG1 (N= 18) & EG2 (N=19) & EG3 (N=14) & KG1 (N=17) & N \\
\hline männlich & $7(38,9 \%)$ & $7(36,8 \%)$ & $6(42,9 \%)$ & $8(47 \%)$ & 28 \\
\hline weiblich & $11(61,1 \%)$ & $12(63,2 \%)$ & $8(57,1 \%)$ & $9(53 \%)$ & 40 \\
\hline Alter & $6(33,3 \%)$ & $3(15,8 \%)$ & $2(14,3 \%)$ & $4(23,5 \%)$ & 15 \\
\hline $6-7,11$ & $6(33,3 \%)$ & $7(36,8 \%)$ & $5(35,7 \%)$ & $4(23,5 \%)$ & 22 \\
\hline $8-9,11$ & $4(22,2 \%)$ & - & $4(28,6 \%)$ & $4(23,5 \%)$ & 12 \\
\hline $10-11,11$ & $2(11,1 \%)$ & $8(42,1 \%)$ & $2(14,3 \%)$ & $2(11,8 \%)$ & 14 \\
\hline $12-13,11$ & - & $1(5,3 \%)$ & $1(7,1 \%)$ & $3(17,7 \%)$ & 5 \\
\hline Ab 14
\end{tabular}

Der Anteil der 12 - 13,11jährigen ist in der EG2, also in der Gruppe mit frühem Betreuungswechsel, gegenüber den anderen Gruppen deutlich erhöht, dafür ist keines der Kinder dieser Gruppe zwischen 10 und 11,11 Jahren. Das durchschnittliche Alter beträgt in den vier Gruppen 9 Jahre, 1 Monat (EG1), 10 Jahre, 5 Monate (EG2), 10 Jahre, 2 Monate (EG3) und 10 Jahre, 5 Monate (KG1).

\section{Schulbesuch}

Aus Tabelle 8.20 ist ersichtlich, welche Schule die Kinder der einzelnen Gruppen jeweils besuchten. In allen vier Gruppen bilden die Grundschüler die anteilsmäßig größte Gruppe. Kinder der Gruppen EG2, EG3 und KG1 besuchen weiterführende Schulen, nicht aber die Kinder der EG1, was durch das etwas niedrigere Durchschnittsalter bedingt ist. Ein paar Kinder besuchten aufgrund ihres jungen Alters noch gar keine Schule. 
Tab. 8.20 Besuchte Schulformen

\begin{tabular}{|l|c|c|c|c|}
\hline Schulform & EG1 $(\mathbf{N}=\mathbf{1 8})$ & EG2 $(\mathbf{N}=\mathbf{1 9})$ & EG3 (N= 14) & KG1 ( N = 17) \\
\hline Grundschule & $10(55,6 \%)$ & $10(52,6 \%)$ & $9(64,3 \%)$ & $9(52,9 \%)$ \\
\hline Orientierungsstufe & $2(11,1 \%)$ & - & $1(7,1 \%)$ & $1(5,9 \%)$ \\
\hline Hauptschule & - & $2(10,5 \%)$ & - & $1(5,9 \%)$ \\
\hline Realschule & - & $2(10,5 \%)$ & $1(7,1 \%)$ & $1(5,9 \%)$ \\
\hline Gymnasium & - & $2(10,5 \%)$ & $1(7,1 \%)$ & - \\
\hline Schule für Lernhilfe & $2(11,1 \%)$ & $1(5,3 \%)$ & $2(14,3 \%)$ & $1(5,9 \%)$ \\
\hline Schule für Erziehungshilfe & - & $1(5,3 \%)$ & - & - \\
\hline Schule für Praktisch Bildbare & - & - & - & $1(5,9 \%)$ \\
\hline Sprachheilschule & $1(5,55 \%)$ & - & - & - \\
\hline Vorklasse & $1(5,55 \%)$ & - & - & - \\
\hline Kein Schulbesuch & $1(5,55 \%)$ & $1(5,3 \%)$ & - & $1(5,9 \%)$ \\
\hline unbekannt & $1(5,55 \%)$ & & & $2(11,8 \%)$ \\
\hline
\end{tabular}

\subsubsection{HIV-Status der Kinder}

Die Zahl der HIV-Infektionen der Kinder veranschaulicht Tabelle 8.21. Das Risiko einer Übertragung der HIV-Infektion der Mutter während der Geburt ist mittlerweile sehr gering, so dass trotz der relativ großen Zahl an Infektionen unter den leiblichen Müttern insgesamt nur 4 Kinder HIV-positiv sind.

Tab. 8.21 HIV-Infektion der Kinder

\begin{tabular}{|l|c|c|c|c|}
\hline & EG1 $(\mathrm{N}=18)$ & EG2 $(\mathrm{N}=19)$ & EG3 $(\mathrm{N}=14)$ & KG1 $(\mathrm{N}=17)$ \\
\hline HIV-Infektion des Kindes & 1 & 1 & 0 & 2 \\
\hline
\end{tabular}




\subsection{Untersuchungsinstrumente}

\subsubsection{Der Datenerfassungsbogen}

Der Datenerfassungsbogen (KDAM-Bogen) beruht im Wesentlichen auf der „Basisdokumentation für Kinder und Jugendliche“ der Abteilung für Kinder und Jugendpsychiatrie der Johann-Wolfgang-Goethe-Universität, Frankfurt a. Main (Englert \& Poustka, 1995) und ist bei Ziegler (1998) detailliert dargestellt. Er umfasst Fragen zu Daten von Mutter und Kind, der sozialen Situation, dem Drogenkonsum, Verlauf von Schwangerschaft und Geburt, der Entzugssymptomatik, dem postpartalen Verlauf und dem Fürsorgeverhalten der Familie. Die Daten wurden von Ziegler aus Krankenakten und Aufzeichnungen der Sozialstation retrospektiv erhoben und in den KDAM-Bogen übertragen.

\subsubsection{Interviews und Testverfahren bei Kindern}

- Psychosoziale Belastung der Kinder: Das standardisierte Elterninterview zur Achse V (Aktuelle abnorme psychosoziale Umstände; Poustka. Burk, Bästlein, van Goor-Lambo \& Shermer, 1994; Bästlein \& Poustka, 1993) erfasst die psychosoziale Belastung der Kinder. Die hier verwendete Lifetime-Version wurde aus einem früheren semistrukturierten Elterninterview abgeleitet und dient der Erfassung der psychosozialen Belastungen des Kindes/des Jugendlichen sowohl in früheren Lebensabschnitten als auch in der Gegenwart. Entwickelt wurde das Interview nach dem Glossar zur Achse V des Multiaxialen Klassifikationsschemas für psychiatrische Errankungen des Kindes- und Jugendalters. Seit ihrer Revision im Jahr 1994 gliedert sich die Achse V in neun Hauptkategorien mit insgesamt 39 Subkategorien chronischer inner- und außerfamiliärer, akuter und störungsabhängiger psychosozialer Belastungen. Untersuchungen zeigen, dass bei guter Kenntnis des Glossars und strikter Einhaltung der Kodierungskriterien die verlässliche und valide Erfassung der Belastungsfaktoren möglich ist (Poustka, 1993; Overmeyer, Schmidt \& Blanz, 1993).

- Psychopathologie der Kinder: Die Child Behavior Checklist (CBCL, Döpfner et al., 1994). Dieser international eingesetzte, im Normalfall von den Eltern ausgefüllte Fremdbeurteilungsbogen, besteht aus insgesamt 113 Items, die sich in insgesamt 8 Subskalen gliedern. Diese Skalen können zu einer Skala Externalisierend (Dissoziales und Aggressives Verhalten) und zu einer Skala Internalisierend (Sozialer Rückzug, Körperliche Beschwerden, 
Angst/Depression) zusammengefasst werden. Drei Skalen, nämlich Soziale Probleme, Schizoid/Zwanghaft und Aufmerksamkeitsprobleme, gehören keiner dieser Gruppen an. Ebenfalls berechnet werden kann ein alle acht Subskalen umfassender Gesamtscore.

Neben der CBCL wird zur Erfassung der kindlichen Psychopathologie das ,Schedule for Affective Disorders and Schizophrenia“ (K-SADS) in der deutschen Fassung für Kinder und Jugendliche (Delmo et al., 1998) verwendet. Es handelt sich um ein semistrukturiertes diagnostisches Interview zur Erfassung gegenwärtiger und zurückliegender Episoden psychischer Störungen. Das Interview wird sowohl mit den Eltern als auch mit den Kindern durchgeführt, aus diesen Informationen und dem klinischen Eindruck wird eine zusammenfassende Beurteilung generiert. Das Kiddie-SADS besteht aus dem unstrukturierten Eingangsinterview, dem Screening-Interview und insgesamt fünf diagnostischen Erweiterungsinterviews, die aber im Falle positiver Kodierungen im Screeningteil zum Einsatz kommen. Innerhalb dieses Interviews wird auch ein eventueller Nikotin-, Alkoholund Drogenkonsum erhoben.

- Psychosoziale Anpassung der Kinder: Die Global Assessment of Functioning Scale Globales psychosoziales Funktionsniveau (GAF; APA, 1989) wurde ursprünglich von Shaffer für Kinder und Jugendliche adaptiert (Children's Global Assessment Scale, CGAS) und auf ihre Reliabilität und Validität überprüft (Shaffer et al, 1983).

Die Einschätzung des psychosozialen Funktionsniveaus (Achse VI) soll die psychische, soziale und schulische Leistungsfähigkeit des Kindes/des Jugendlichen widerspiegeln. Die Untersuchungsteilnehmer werden in der hier verwendeten Skala von 0 (Herausragende/gute soziale Funktionen) bis 8 (Tiefe und durchgängige soziale Beeinträchtigung) bewertet. Das Scoringschema befindet sich im Anhang.

- Allgemeine Intelligenz der Kinder: Hamburg-Wechsler-Intelligenztest für Kinder-III (HAWIK-III, Tewes, Rossmann \& Schallberger, 1999). Der HAWIK-III ist einsetzbar für Kinder und Jugendliche von 6,0 bis 16,11 Jahren. Er besteht aus insgesamt 11 Untertests, bzw. wurde der HAWIK-III um zwei optionale Untertests erweitert, die in dieser Untersuchung aber nicht angewendet wurden. Neben den Skalen der Subtests werden die praktische, die verbale und die allgemeine Intelligenz im Sinne des Globalkonzepts von Wechsler erfasst (Tewes et al., 1999). Die Durchführungsdauer beträgt ca. 90 Minuten. 


\subsubsection{Interviews und Testverfahren bei Müttern/Bezugspersonen}

- Psychopathologie der Mütter: Adult Self Report (ASR, Arbeitsgruppe deutsche CBCL, 1997). Dieser international verwendete Selbstbeurteilungsbogen besteht aus insgesamt 137 Items, die sich in insgesamt 8 Subskalen gliedern. Diese Skalen können zu einer Skala Externalisierend (Aggressives Verhalten, Regelverletzendes Verhalten, Intrusives Verhalten) und zu einer Skala Internalisierend (Rückzug, Körperliche Beschwerden, Angst/Depression) zusammengefasst werden. Zwei Skalen, nämlich Schizoid/Zwanghaft und Aufmerksamkeitsprobleme, gehören keiner dieser Gruppen an. Ebenfalls berechnet wird ein alle acht Subskalen umfassender Gesamtscore.

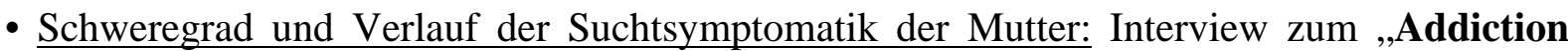
Severity Index“ (ASI, Gsellhofer, Fahrner \& Platt, 1994). Der ASI ist ein innerhalb der Suchtforschung weit verbreitetes semi-standardisiertes Interview, mit dem Informationen über Lebensbereiche abgefragt werden, die in Zusammenhang mit der Substanzabhängigkeit stehen. So beinhaltet der ASI Fragen zum körperlichen Zustand, zu Arbeit/Unterhalt und zu rechtlichen, familiären/sozialen und psychischen Problemen. Der Drogen- und Alkoholgebrauch wird sowohl für die vorangegangenen 30 Tage als auch für die Lebenszeit erfragt. Basierend auf den erfragten Informationen werden für alle Bereiche Schweregradratings vergeben.

\subsection{Untersuchungsdurchführung}

Die Untersuchungen der Mütter (bzw. der Hauptbezugspersonen) und ihrer Kinder erfolgte in der Regel in der Ambulanz der Kinder- und Jugendpsychiatrie des Klinikums der Johann Wolfgang Goethe-Universität Frankfurt a. Main. In einigen Fällen erfolgte auf Wunsch der Familie auch ein Hausbesuch.

Die Unbedenklichkeit der Studie wurde durch die Ethikkommission der Universität Frankfurt abgesichert und die teilnehmenden Eltern bzw. Bezugspersonen wurden vor Beginn der Studie im Sinne eines ,informed consent“ über Ablauf und Ziel der Untersuchung mittels einer Teilnehmerinformation unterrichtet. In einem ersten einleitenden Teil, der mit Eltern und Kind gemeinsam durchgeführt wurde, war oft eine besondere Sensibilität notwendig, da viele Kinder über die Hintergründe der Untersuchung nicht vollständig Bescheid wussten. So gab es einzelne Fälle HIV-positiver Kinder, die über ihre Infektion nicht informiert waren und 
einige der Kinder, die bei Adoptiveltern oder Großeltern aufwuchsen, wussten z.B. nichts über die Drogenerkrankung ihrer leiblichen Mütter oder die Todesumstände eines oder beider Elternteile.

Bis auf wenige Ausnahmen, bei denen ein Abweichen aus verschiedenen Gründen notwendig erschien, wurde die Untersuchung mit einigen „warming-up“ Fragen an das Kind bezüglich Schule, Hobbys, Freunde begonnen. Danach erfolgte der Screening-Teil des Kiddie-SADS und bei positiver Kodierung schlossen sich die entsprechenden Erweiterungsinterviews daran an. Bei den kleineren Kindern wurde das Interview mit den Eltern gemeinsam durchgeführt, bei den älteren Kindern, ab ca. einem Alter von 10 oder 11 Jahren erfolgte das Interview getrennt, wobei zuerst das Kind und dann die Mutter befragt wurde. Nach Abschluss des Kiddie-SADS wurde von der an der Untersuchung mitarbeitenden wissenschaftlichen Hilfskraft der HAWIK-III durchgeführt, während zeitgleich die Eltern anhand des Interviews zur Achse V zu den psychosozialen Umständen befragt wurden. Den nächsten Abschnitt der Untersuchung bildete der Addiction Severity Index. Dieses suchtspezifische Interview wurde innerhalb der EG zunächst nur mit den leiblichen Müttern durchgeführt, bzw. am Anfang mit den Hauptbezugspersonen über die leibliche Mutter. Mit fortschreitender Dauer des Projektes zeichnete sich immer mehr ab, dass der Anteil der Adoptiv- und Pflegeeltern groß sein würde, weshalb im Hinblick auf die Vollständigkeit der Daten das Interview auch mit ihnen durchgeführt wurde. Bei den meisten Pflege- und Adoptiveltern, bei denen das zu Beginn des Projektes versäumt worden war, konnte das Interview telefonisch nachgeholt werden. Wann immer möglich füllten die Bezugspersonen die Fragebögen CBCL und ASR innerhalb der Untersuchung in der Klinik aus. In manchen Fällen wurden die Bögen mit einem Rückantwortkuvert aber auch zum Ausfüllen mit nach Hause gegeben. Von den Kindern wurden die Fragebögen immer in der Klinik ausgefüllt. Nach Beendigung der Untersuchung wurden den Bezugspersonen 100 DM, bzw. ab $200250 €$ Aufwandsentschädigung bar ausbezahlt. Zwei Familien hatten längere Anfahrtswege und eine dieser Familien musste auf Grund der großen Entfernung zum Heimatort auch in Frankfurt übernachten. Die dafür entstandenen Kosten wurden aus Projektmitteln erstattet.

\subsection{Untersuchungsdesign}

Bei der Frage nach der Entwicklung der Kinder soll sowohl der Einfluss der biologischen Vulnerabilität als auch des psychosozialen Risikos berücksichtigt werden. Die bereits beschriebene Stichprobeneinteilung in EG1, EG2, EG3 und KG1 wurde im Hinblick auf die 
postnatalen Erziehungsbedingungen vorgenommen. Das spezifische Risiko der Opiatexposition besteht bei den Gruppen EG1, EG2, EG3. Bei allen vier Gruppen besteht ein unspezifisches Risiko aufgrund medizinischer Komplikationen und Risikofaktoren in Bezug auf Geburtsgewicht und Gestationsalter.

Mit den Kriterien wird die kognitive und die sozial-emotionale Entwicklung der Kinder erfasst.

Die Einflussvariablen gliedern sich in kindliche und mütterliche Variablen sowie eine spezifisch für die Gruppe der opiatexponierten Kinder wirksame Variable.

\subsubsection{Kriterien}

Die Entwicklung der Kinder wird anhand folgender Kriterien (Outcomevariablen) untersucht:

- Kognitive Entwicklung: Gesamtscore, Verbal-IQ und Handlungs-IQ sowie die einzelnen Subskalen des HAWIK-III.

- Psychopathologie der Kinder: Gesamtscore der CBCL, die Skalenwerte Externalisierend und Internalisierend sowie die einzelnen Subskalen (T-Werte).

- Psychosoziale Anpassung: Ausprägungsgrad auf der Global Assessment of Functioning Scale.

\subsubsection{Einflussfaktoren}

Im Folgenden werden alle Variablen aufgelistet, die einen potentiell beeinflussenden bzw. moderierenden Effekt auf die Kriterien haben. Als Einflussvariable des Kindes nicht berücksichtigt wurde aufgrund ihrer sehr kleinen Auftretenszahl (siehe Tabelle 8.21) die HIVInfektion des Kindes.

\section{Einflussvariablen auf Seiten des Kindes sind:}

- Geschlecht des Kindes

- Alter des Kindes in Monaten: (zum Zeitpunkt der Untersuchung)

- Geburtsgewicht des Kindes: (in Gramm)

- Gestationsalter des Kindes: (in Wochen) 
- Psychosoziale Belastung des Kindes (Achse V): Als Maß für die psychosoziale Belastung wurde ein Gesamtscore über alle Belastungssituationen gebildet, der sich aus der ungewichteten Summe der Kodierungen mit „1“ (entsprechende Belastung liegt in klinisch abnormer Weise vor, der Schweregrad entspricht aber nicht dem geforderten Ausmaß) und der Kodierungen mit „2“ (Belastung liegt im spezifizierten Zeitraum mit Sicherheit und den diagnostischen Kriterien eindeutig genügendem Schweregrad vor) in den einzelnen Kodierungsabschnitten (0 - 12 Monate, 1. - 3. Lebensjahr, 4. - 6. Lebensjahr, 7. - 9. Lebensjahr, 10. - 12. Lebensjahr, 13.-15. Lebensjahr und 16. - 18. Lebensjahr) errechnet.

Wenn die psychosoziale Situation als unauffällig, d.h. ohne klinische Relevanz bewertet wurde, erfolgte eine Kodierung mit “ $0 “ ., „ 8 “$ bedeutete, dass die Situation für das jeweilige Kind logisch nicht möglich war (z.B. Fragen zu Geschwistern bei Einzelkindern); eine Kodierung mit „9“ kam zum Tragen, wenn die Informationen zur Einschätzung der Situation nicht ausreichend waren.

- Betreuungswechsel: Die Anzahl der Betreuungswechsel ist eine spezifische Variable der psychosozialen Bedingungen und genauso wie die psychosoziale Belastung an sich definierend für die Gruppenteinteilung.

\section{$\underline{\text { Zu den Einflussvariablen auf Seiten der Mutter gehören: }}$}

- Psychopathologie der Bezugsperson: Als Parameter diente der Gesamtscore (T-Werte) des Adult Self Report (ASR).

- Schulbildung der Bezugsperson: Die Schulbildung der Bezugsperson wurde als kontinuierliche Variable behandelt.

SB1: kein Schulabschluss (1)

SB2: Hauptschule (2)

SB3: Mittlere Reife (3)

SB4: Abitur (4)

SB5: Berufsfachschule, Akademie (5)

SB6: Hochschulabschluss (6)

Diese Einflussvariablen liegen für alle Bezugspersonen vor, sind aber nur in Bezug auf die Gruppen EG1, EG2 und KG1 sinnvoll zu verwenden, nicht aber für die Gruppe EG3, da sich hier aufgrund des späten und teilweise mehrfachen Wechsel keine konstante 
Hauptbezugsperson ermitteln lässt und so die Zeitspanne des Einflusses dieser mütterlichen Variablen sehr stark variiert.

\section{Suchtspezifische Einflussvariablen}

Eine spezifisch für die Suchtgruppe wirksame Variable ist die

- Schwere des Entzugs (Score nach Finnegan): Als Maß für die Schwere der Entzugssymptomatik wurde aus den Daten von Ziegler der Summenscore zur Einschätzung des Abstinenzsyndroms (in Anlehnung an den Therapiescore nach L. Finnegan, 1985) übernommen. Es kann maximal ein Punktwert von 33 erreicht werden.

- Substitution

\subsubsection{Versuchsplan}

1. In einem ersten Schritt werden die oben aufgelisteten Einflussfaktoren für die Gruppen EG1, EG2, EG3 und KG1 beschrieben und es wird überprüft, ob sich die einzelnen Gruppen hinsichtlich dieser Parameter signifikant unterscheiden. Aus den Ergebnissen zur Variable der „psychosozialen Belastung“ erfolgt die Überprüfung der Hypothese I.

2. Die Beziehung zwischen Kriterien und potentiell konfundierenden Variablen wird anhand von korrelationsstatistischen Verfahren überprüft, und zwar getrennt für die Gesamtgruppe der opiatexponierten Kinder EG (EG1, EG2, EG3) und für die KG1. Die Hypothese II, zum Einfluss der Schwere des Abstinenzsyndroms auf die kognitive Entwicklung, lässt sich aus den korrelationsstatistischen Ergebnissen überprüfen.

Für die Einflussvariablen der Bezugspersonen, Psychopathologie und Schulbildung, wird eine für die leiblichen Mütter (EG1 und KG1) getrennte Korrelationstabelle erstellt, da nur bei diesen beiden Gruppen die gleichen Bedingungen bestehen (genetischer Einfluss, Einfluss von Geburt an) und somit eine sinnvolle Interpretation möglich ist.

3. Für die Beantwortung der Hypothesen III und IV erfolgt ein Gruppenvergleich hinsichtlich der Kriterien mittels Kovarianzanalysen. Die Begründung für die Auswahl der Kovariaten wird in Abschnitt 9.2.4 beschrieben, bzw. richtet sich im Fall der körperlichen Einflussvariablen der Kinder nach den Ergebnissen aus den Schritten 1 und 2.

4. Um den Effekt des biologischen Risikos und des psychosozialen Risikos in Bezug auf die einzelnen Kriterien zu untersuchen werden Regressionsanalysen gerechnet. 


\subsubsection{Statistische Verfahren}

Zur Berechung von Verteilungsunterschieden werden ?2-Tests und zur Berechnung von Mittelwertsunterschieden wurden Varianzanalysen, bzw. bei Berücksichtigung potentiell konfundierender Variablen Kovarianzanalysen durchgeführt. Auf die Durchführung des parameterfreien Verfahrens Kruskal-Wallis-Test, der normalerweise eingesetzt wird, wenn die Voraussetzungen für die Varianzanalyse nicht erfüllt sind, nämlich einerseits die Homogenität der Varianzen, andererseits die Normalverteilung innerhalb der Gruppen, wurde verzichtet, da bei unseren Analysen die Stichproben annähernd gleich groß waren und in diesem Fall das Verfahren der Varianzanalyse gegenüber Verletzungen der Voraussetzungen robust ist (Bortz, 1993). Bei einem signifikanten Ergebnis (Signifikanzniveau $\alpha=0,05$ ) wurden die nachfolgenden paarweisen Mittelwertsvergleiche im Fall der Varianzanalyse mit der Scheffé-Prozedur berechnet. Im Fall einer Kovarianzanalyse wurden die nachfolgenden paarweisen Gruppenvergleiche ebenfalls mittels Kovarianzanalyse berechnet. 


\section{ERGEBNISSE}

\subsection{Prädiktoren/Einflussfaktoren}

Im Folgenden sind die Einflussvariabeln für die Gruppen EG1, EG2, EG3 und KG1 aufgeführt. Angegeben sind Mittelwert (MW) und Standardabweichung (SD) sowie gegebenenfalls die Zahl der Fälle oder Prozentsätze.

\subsubsection{Somatische Variablen der Kinder}

Tab. 9.1 Einflussvariablen der Kinder

\begin{tabular}{|c|c|c|c|c|c|c|c|}
\hline & EG1 & EG2 & EG3 & KG1 & $\mathbf{F}$ & Chi & Sig. \\
\hline \multicolumn{8}{|c|}{ Körperliche Variablen der Kinder } \\
\hline Geschlecht & $(\mathrm{N}=18)$ & $(\mathrm{N}=19)$ & $(\mathrm{N}=14)$ & $(\mathrm{N}=17)$ & & & \\
\hline Männlich & 7 & 7 & 6 & 8 & & & \\
\hline Weiblich & 11 & 12 & 8 & 9 & & 0,445 & 0,931 \\
\hline Alter (in Monaten) & $(\mathrm{N}=18)$ & $(\mathrm{N}=19)$ & $(\mathrm{N}=14)$ & $(\mathrm{N}=17)$ & & & \\
\hline $\begin{array}{l}\mathrm{MW} \\
(\mathrm{SD})\end{array}$ & $\begin{array}{c}109,4 \\
(24,03) \\
\end{array}$ & $\begin{array}{c}125 \\
(30,12) \\
\end{array}$ & $\begin{array}{c}122 \\
(24,39) \\
\end{array}$ & $\begin{array}{c}125,3 \\
(38,76) \\
\end{array}$ & 1,107 & & 0,353 \\
\hline Geburtsgewicht (Gramm) & $(\mathrm{N}=16)$ & $(\mathrm{N}=14)$ & $(\mathrm{N}=13)$ & $(\mathrm{N}=15)$ & & & \\
\hline $\begin{array}{l}\text { MW } \\
(\mathrm{SD})\end{array}$ & $\begin{array}{c}2885 \\
(422,25) \\
\end{array}$ & $\begin{array}{c}2649 \\
(493,64) \\
\end{array}$ & $\begin{array}{c}2668 \\
(750,25) \\
\end{array}$ & $\begin{array}{c}2873 \\
(1021,52) \\
\end{array}$ & 0,469 & & 0,705 \\
\hline Gestationsalter (Wochen) & $(\mathrm{N}=15)$ & $(\mathrm{N}=12)$ & $(\mathrm{N}=13)$ & $(\mathrm{N}=15)$ & & & \\
\hline $\begin{array}{l}\text { MW } \\
\text { (SD) }\end{array}$ & $\begin{array}{l}38,80 \\
(1,42)\end{array}$ & $\begin{array}{l}37,17 \\
(1,27)\end{array}$ & $\begin{array}{l}37,69 \\
(3,17)\end{array}$ & $\begin{array}{l}37,27 \\
(3,97)\end{array}$ & 1,059 & & 0,375 \\
\hline
\end{tabular}

Beim Vergleich der Subgruppen bestehen, wie aus Tabelle 9.1 ersichtlich, hinsichtlich Geschlecht, Alter, Geburtsgewicht und Gestationsalter keine Verteilungsunterschiede.

\subsubsection{Psychosoziale Faktoren}

\section{$\underline{\text { Achse V }}$}

Tabelle 9.2 zeigt, dass sich bei der psychosozialen Belastung deutliche Gruppenunterschiede finden. Die Gruppe EG3 weist eine signifikant stärkere Belastung auf als die Gruppen EG1, EG2 und KG1. Deskriptiv betrachtet besteht bei der KG1 die niedrigste Belastung. 
Tab. 9.2 Gesamtscore der psychosozialen Belastung

\begin{tabular}{|c|c|c|c|c|c|c|}
\hline & $\operatorname{EG1}(\mathrm{N}=18)$ & EG2 (N = 19) & EG3 $(N=14)$ & KG1 $(N=17)$ & $\mathbf{F}$ & Sig. \\
\hline \multicolumn{7}{|c|}{ Psychosoziale Belastung Achse V } \\
\hline $\begin{array}{l}\text { MW } \\
\text { (SD) }\end{array}$ & $\begin{array}{c}9,5 \\
(1,9)\end{array}$ & $\begin{array}{c}8,1 \\
(3,9)\end{array}$ & $\begin{array}{l}14,8 \\
(4,0)\end{array}$ & $\begin{array}{c}6,6 \\
(4,6)\end{array}$ & 13,255 & 0,000 \\
\hline
\end{tabular}

In der nachfolgenden Tabelle 9.3 werden die einzelnen Kategorien der Achse $\mathrm{V}$ zur Veranschaulichung der Qualität der psychosozialen Belastung aufgeführt. Das Vorhandensein einer Belastung wurde als gegeben angenommen, wenn die entsprechende Kategorie mit „1“ oder mit „2“ kodiert wurde. Für eine positive Kodierung reichte das Vorhandensein der Belastung zu irgendeinem Zeitpunkt der gesamten Lebenszeit.

Tab. 9.3 Qualität der psychosozialen Belastung

\begin{tabular}{|c|c|c|c|c|}
\hline Variable & $\begin{array}{c}\text { EG1 } \\
(\mathbf{N}=17)\end{array}$ & $\begin{array}{c}\text { EG2 } \\
(\mathbf{N}=19)\end{array}$ & $\begin{array}{c}\text { EG3 } \\
(\mathbf{N}=14)\end{array}$ & $\begin{array}{c}\text { KG1 } \\
(\mathbf{N}=15)\end{array}$ \\
\hline \multicolumn{5}{|l|}{5 Abnorme unmittelbare Umgebung } \\
\hline 5.0 Erziehung in einer Institution & $1(5,8 \%)$ & $2(10,5 \%)$ & $8(57,1 \%)$ & 0 \\
\hline 9.0 Institutionelle Erziehung & 0 & 0 & 0 & 0 \\
\hline 6.1 Bedrohliche Umstände/Fremdunterbringung & $2(11,8 \%)$ & $6(31,6 \%)$ & $6(42,9 \%)$ & $2(13,3 \%)$ \\
\hline 9.1 Fremdunterbringung durch Symptomatik & 0 & 0 & $2(14,3 \%)$ & 0 \\
\hline 5.1.0 Erziehung durch nichtbiologischen Elternteil & $8(47,1 \%)$ & $19(100 \%)$ & $14(100 \%)$ & $7(46,7 \%)$ \\
\hline 5.1.1 Erzieh. abweichende Familienbedingungen & $2(11,8 \%)$ & $3(15,8 \%)$ & $5(35,7 \%)$ & 0 \\
\hline \multicolumn{5}{|l|}{$\begin{array}{l}8 \text { Chronische zwischenmenschliche Belastung/ in } \\
\text { Schule oder Arbeit }\end{array}$} \\
\hline 8.0 Streitbeziehung mit Schülern & $1(5,8 \%)$ & $5(26,3 \%)$ & $5(35,7 \%)$ & $1(6,7 \%)$ \\
\hline 8.1 Negative Auseinandersetzungen mit Lehrern & $3(17,6 \%)$ & $2(10,5 \%)$ & $5(35,7 \%)$ & $2(13,3 \%)$ \\
\hline 8.2 Allgemeine Unruhe in der Schule & $1(5,8 \%)$ & $2(10,5 \%)$ & $1(7,1 \%)$ & $1(6,7 \%)$ \\
\hline 7.1 Migration oder soziale Verpflanzung - akt. & $7(41,2 \%)$ & $6(31,6 \%)$ & $7(50 \%)$ & $5(33,3 \%)$ \\
\hline 5.2 Isolierte Familie & $7(41,2 \%)$ & $1(5,3 \%)$ & $6(42,9 \%)$ & $1(6,7 \%)$ \\
\hline 5.3 Psychosoziale Gefährdung & $14(82,4 \%)$ & $4(21,1 \%)$ & $14(100 \%)$ & $7(46,7 \%)$ \\
\hline \multicolumn{5}{|l|}{6 Akute. Belastende Lebensereignisse } \\
\hline 6.0 Verlust einer liebevollen Beziehung & $12(70,6 \%)$ & $9(47,4 \%)$ & $13(92,9 \%)$ & $10(66,6 \%)$ \\
\hline 6.2 Neg. Veränderung neue Familienmitglieder - akt. & $3(17,6 \%)$ & $3(15,8)$ & $4(28,6 \%)$ & $3(20 \%)$ \\
\hline 6.3 Unabh. Ereignisse - geringe Selbstachtung & $3(17,6 \%)$ & $9(47,4 \%)$ & $8(57,1 \%)$ & $1(6,7 \%)$ \\
\hline 9.2 Abh. Ereignisse - geringe Selbstachtung & $1(5,9 \%)$ & $2(10,5 \%)$ & 0 & $1(6,7 \%)$ \\
\hline \multicolumn{5}{|l|}{7 Gesellschaftliche Belastungsfaktoren } \\
\hline 7.0 Verfolgung oder Diskriminierung & 0 & 0 & $1(7,1 \%)$ & $1(6,7 \%)$ \\
\hline 6.5 Unmittelbare, beängstigende Ereignisse & $1(5,9 \%)$ & $4(21,1 \%)$ & $4(28,6 \%)$ & $2(13,3 \%)$ \\
\hline 6.4 Sexueller Missbrauch außerhalb d. Familie & 0 & 0 & $2(14,3 \%)$ & 0 \\
\hline
\end{tabular}


Tab. 9.3 (Fortsetzung) Qualität der psychosozialen Belastung

\begin{tabular}{|c|c|c|c|c|}
\hline \multicolumn{5}{|l|}{4 Abnorme Erziehungsbedingungen } \\
\hline 4.0 Elterliche Überfürsorge - akt. & $1(5,8 \%)$ & $4(21,1 \%)$ & $2(14,3 \%)$ & $2(13,3 \%)$ \\
\hline 4.1 Unzureichende elterliche Aufsicht & $9(52,9 \%)$ & $6(31,6 \%)$ & $10(71,4 \%)$ & $2(13,3 \%)$ \\
\hline 4.2 Erziehung mit unzureichender Erfahrung & $7(41,2 \%)$ & $5(26,3 \%)$ & $9(75 \%)$ & $2(13,3 \%)$ \\
\hline 4.3 Unangemessene Anforderungen & 0 & $3(15,8 \%)$ & $5(35,7 \%)$ & $2(13,3 \%$ \\
\hline \multicolumn{5}{|l|}{1 Abnorme intrafamiliäre Beziehungen } \\
\hline 1.0 Mangel an Wärme in der Eltern-Kind-Bez. & $2(11,8)$ & $4(21,1 \%)$ & $6(42,9 \%)$ & $2(13,3 \%)$ \\
\hline 1.1 Disharmonie in der Familie zw. Erwachsenen & $14(82,4 \%)$ & $7(36,8 \%)$ & $11(78,6 \%)$ & $9(60 \%)$ \\
\hline 1.2 Feindliche Ablehnung geg. dem Kind & $1(5,8 \%)$ & $3(15,8 \%)$ & $2(14,3 \%)$ & $1(6,7 \%)$ \\
\hline 1.3 Körperliche Kindesmisshandlung - akt. & $1(5,8 \%)$ & $3(15,8 \%)$ & $3(21,4 \%)$ & $1(6,7 \%)$ \\
\hline 1.4 Sexueller Missbrauch in der Familie & 0 & 0 & $2(14,3 \%)$ & 0 \\
\hline \multicolumn{5}{|l|}{3 Inadäquate intrafamiliäre Kommunikation } \\
\hline 3 Inadäquate Kommunikation & $3(17,6 \%)$ & $6(31,6 \%)$ & $4(28,6 \%)$ & $3(20 \%)$ \\
\hline \multicolumn{5}{|l|}{2 Psych. Störung/Behinderung in Familie - akt. } \\
\hline 2.0 Psych. St./abweichendes Verh. d. Eltern & $15(88,2 \%)$ & $8(42,1 \%)$ & $11(78,6 \%)$ & $4(26,7 \%)$ \\
\hline 2.1 Behinderung eines Elternteils & $8(50 \%)$ & $2(10,5 \%)$ & $11(78,6 \%)$ & $4(26,7 \%)$ \\
\hline 2.2 Behinderung der Geschwister. & $3(30 \%)$ & $1(11,1 \%)$ & $1(10 \%)$ & $1(11,1 \%)$ \\
\hline
\end{tabular}

Die insgesamt große Belastung der Gruppe EG3 ist vor allem durch eine hohe Belastung in den Kategorien bedingt, die direkt oder indirekt mit der Zuordnung in die Gruppe EG3 zusammenhängen. Dabei kann es sich sowohl um mögliche Ursachen der Fremdunterbringung (z.B. 4.1 Unzureichende elterliche Aufsicht; 4,2 Erziehung die eine unzureichende Erfahrung vermittelt; 1.1 Disharmonie in der Familie, 5.3 Psychosoziale Gefährdung) als auch um mögliche oder auch unausweichliche Folgen einer Fremdunterbringung handeln (z.B. 5.0 Erziehung in einer Institution; 5.1.0 Erziehung durch nichtbiologischen Elternteil; 6.0 Verlust einer liebevollen Beziehung....). Auch in der EG2 sind diese Kategorien teilweise $\mathrm{zu}$ hohen Prozentsätzen vertreten, allerdings trotzdem insgesamt zu einem geringeren Grad als in der EG3. Spezifische Belastungen in der EG1 im Vergleich zu den ebenfalls durch die leiblichen Eltern versorgten Kinder der KG1 sind als Folge des Aufwachsens in einem suchtspezifischen Milieu und dadurch bedingten erhöhten Risiken zu verstehen: Dazu gehört ebenfalls eine erhöhte psychosoziale Gefährdung (5.3), Disharmonie in der Familie (1.1) oder auch Psychische Störung/abweichendes Verhalten der Eltern (2.0). Der letztgenannte Punkt ist per se durch die Suchterkrankung der Eltern in EG1 deutlich höher als in KG1. 


\section{Anzahl der Betreuungswechsel}

Die Anzahl der Betreuungswechsel ist definitionsgemäß in der Gruppe EG3 am höchsten, und zwar signifikant höher als in allen anderen Gruppen. Durchschnittlich erlebte jedes Kind in der Gruppe EG3 2,64 mal einen Wechsel der Hauptbezugsperson.

Tab. 9.4 Anzahl der Betreuungswechsel

\begin{tabular}{|l|c|c|c|c|c|c|}
\hline & EG1 (N = 18) & EG2 (N = 19) & EG3 (N = 14) & KG1 (N = 17) & F & Sig. \\
\hline \multicolumn{7}{|l|}{ Anzahl der Betreuungswechsel } \\
\hline MW (SD) & 0 & $0,74(0,65)$ & $2,64(1,28)$ & $0,058(0,24)$ & 47,716 & 0,000 \\
\hline
\end{tabular}

\subsubsection{Zusammenhang von Gruppenzugehörigkeit und psychosozialer Belastung (Hypothese I)}

Aus den Ergebnissen des Abschnitts 9.1.2 lässt sich eindeutig feststellen, dass die psychosoziale Belastung in der Gruppe mit den meisten Betreuungswechseln (EG3) am stärksten ist und signifikant größer als in allen anderen Gruppen. Hypothese I ist damit bestätigt.

\subsubsection{Einflussfaktoren der Mütter/Bezugspersonen}

Die Einflussfaktoren der Mütter/Bezugspersonen beziehen sich immer auf die aktuelle Hauptbezugsperson. In den Gruppen EG1 und KG1 sind das die leiblichen Mütter, in den Gruppen EG2 und EG3 Großmütter, Pflege- oder Adoptiveltern.

\subsubsection{Psychopathologie der Mütter/Bezugspersonen}

Aus Tabelle 9.5 sind die durchschnittlichen Skalenwerte (T-Werte) des Adult Self Report (ASR) der Mütter und Bezugspersonen ersichtlich.

Deskriptiv betrachtet weisen die leiblichen Mütter der Experimentalgruppe in allen Skalen die stärkste Belastung auf. In den Skalen Sozialer Rückzug, Aufmerksamkeitsprobleme, Regelverletzendes Verhalten, Externalisierend und Internalisierend, sowie in der ASR Gesamtskala besteht ein statistisch signifikanter Unterschied zu den Müttern der EG2. In der Skala Tabakkonsum unterscheiden sich die Mütter der EG1 signifikant zu allen anderen, in der Skala Substanzkonsum-Gesamt besteht ein signifikanter Unterschied zu den Müttern der Gruppen EG2 und EG3. 
Tab. 9.5 Adult Self Report (ASR)

\begin{tabular}{|c|c|c|c|c|c|c|}
\hline & $\begin{array}{c}\text { EG1 } \\
(\mathbf{N}=17)\end{array}$ & $\begin{array}{c}\text { EG2 } \\
(\mathrm{N}=18)\end{array}$ & $\begin{array}{c}\text { EG3 } \\
(\mathbf{N}=13) \\
\end{array}$ & $\begin{array}{c}\text { KG1 } \\
(\mathrm{N}=12) \\
\end{array}$ & & \\
\hline & $\mathrm{MW}(\mathrm{SD})$ & MW (SD) & MW (SD) & $\mathrm{MW}(\mathrm{SD})$ & F (p) $?^{2}$ & \\
\hline \multicolumn{7}{|c|}{ ASR/18-59 Psychopathologie (T-Werte) } \\
\hline Angst/Depression & $60,0612,4)$ & $52,9(5,8)$ & $54,6(5,9)$ & $54,3(4,7)$ & $2,57(0,063) 0,121$ & \multirow{4}{*}{ EG1 > EG2 } \\
\hline Sozialer Rückzug & $60,8(8,7)$ & $52,7(4,7)$ & $56,1(6,7)$ & $53,8(5,9)$ & $4,76(\mathbf{0 , 0 0 5}) 0,203$ & \\
\hline Körp. Beschwerden & $56,4(6,7)$ & $54(6,5)$ & $53,7(8,7)$ & $53(3,3)$ & $0,77(0,516) 0,040$ & \\
\hline Schizoid/Zwanghaft & $55,7(9,1)$ & $51,1(2,6)$ & $52,9(9,6)$ & $52,1(4,3)$ & $1,376(0,260) 0,069$ & \\
\hline Aufmerksam.probleme & $59,2(11,2)$ & $50,9(2,3)$ & $54,5(5,7)$ & $53,3(4,9)$ & $4,214(\mathbf{0 , 0 0 9}) 0,184$ & \multirow[t]{2}{*}{$\mathrm{EG} 1>\mathrm{EG} 2$} \\
\hline Aggressives Verhalten & $58,2(8,8)$ & $53,4(4,5)$ & $55,2(5,9)$ & $54,8(6,2)$ & $0,601(0,199) 0,079$ & \\
\hline Regelverletzendes V. & $61,9(12,9)$ & $51,8(2,7)$ & $55,2(6,3)$ & $54,3(5,9)$ & $4,897(\mathbf{0 , 0 0 4}) 0,208$ & \multirow[t]{2}{*}{$\mathrm{EG} 1>\mathrm{EG} 2$} \\
\hline Intrusives Verhalten & $53,7(6,9)$ & $50,4(0,9)$ & $52,8(3,1)$ & $52,2(4,6)$ & $1,611(0,197) 0,079$ & \\
\hline Externalisierend & $56,4(16,3)$ & $44,7(8,8)$ & $51,5(10,1)$ & $47,9(11,5)$ & $2,910(\mathbf{0 , 0 4 2}) 0,135$ & EG1 > EG2 \\
\hline Internalisierend & $57,9(14,3)$ & $45,3(11,9)$ & $50,6(10,9)$ & $49,5(9,5)$ & $3,272(\mathbf{0 , 0 2 8}) 0,149$ & EG1 > EG2 \\
\hline ASR Gesamtscore & $53,6(16,2)$ & $40,4(10,4)$ & $47,6(11,3)$ & $45(11,8)$ & $3,238(\mathbf{0 , 0 2 9}) 0,148$ & $\mathrm{EG} 1>\mathrm{EG} 2$ \\
\hline \multicolumn{7}{|c|}{ ASR/18-59 Substanzkonsum (T-Werte) } \\
\hline Tabakkonsum & $63(6,6)$ & $53,3(5,6)$ & $54,2(7,9)$ & $55,1(5,1)$ & $7,397(\mathbf{0 , 0 0 0}) 0,295$ & \multirow[t]{4}{*}{$\mathrm{EG} 1>\mathrm{EG} 2, \mathrm{EG} 3, \mathrm{KG} 1$} \\
\hline Alkoholkonsum & $53,5(8,3)$ & $51,7(5,4)$ & $51,6(4,5)$ & $51(3,2)$ & $0,444(0,723) 0,025$ & \\
\hline Drogenkonsum & $57,4(14,2)$ & $50(0)$ & $52,2(7,5)$ & $50(0)$ & $2,521(0,069) 0,134$ & \\
\hline Substanzk. gesamt & $61(8,9)$ & $53,5(6,2)$ & $53,1(6,1)$ & $53,7(3,9)$ & $4,196(\mathbf{0 , 0 1 0}) 0,208$ & \\
\hline
\end{tabular}

\subsubsection{Schulbildung der Bezugspersonen}

Die Verteilung der Schulbildung ist bereits in Abschnitt 8.2.5.1 aufgelistet, wird aber für die bessere Übersichtlichkeit als potentiell relevanter Einflussfaktor nochmals in Tabelle 9.5 angeführt.

Tab. 9.5 Schulbildung der Bezugspersonen

\begin{tabular}{|c|c|c|c|c|c|c|}
\hline & $\begin{array}{c}\text { EG1 } \\
(\mathrm{N}=17)\end{array}$ & $\begin{array}{c}\text { EG2 } \\
(\mathrm{N}=18)\end{array}$ & $\begin{array}{c}\text { EG3 } \\
(\mathrm{N}=13)\end{array}$ & $\begin{array}{c}\text { KG1 } \\
(N=12)\end{array}$ & $?^{2}$ & Sig. \\
\hline \multicolumn{7}{|l|}{ Schulbildung der Bezugsperson } \\
\hline SB1 (kein Abschluss) & & & 1 & 3 & & \\
\hline SB2 (Hauptschule) & 7 & 6 & 2 & 3 & & \\
\hline SB3 (Mittlere Reife) & 5 & 4 & 3 & 6 & & \\
\hline SB4 (Abitur) & 3 & 1 & 2 & 1 & & \\
\hline SB5 (Berufsfachschule, Akademie) & 1 & 5 & 1 & 1 & & \\
\hline SB6 (Hochschulabschluss) & & 2 & 5 & & 28 & 0,022 \\
\hline SB9 (unbekannt) & 2 & 1 & & & & \\
\hline
\end{tabular}

Die Verteilungsunterschiede über alle vier Gruppen betrachtet sind signifikant, wobei vor allem die KG1 im Vergleich zur EG3 einen durchschnittlich geringeren Schulabschluss aufweist - bei den Bezugspersonen der EG3 ist der Hochschulabschluss überrepräsentiert. Berechnet man einen ?2-Test nur für die Gruppen der leiblichen Mütter (EG1 und KG1) so 
finden sich keine signifikanten Verteilungsunterschiede (Pearson $?^{2}=5,582$; df $=4, p=$ $0,233)$.

\subsubsection{Suchtspezifische Einflussfaktoren}

Der größte Teil der Kinder der Experimentalgruppe wies zumindest ein leichtes Entzugssyndrom auf. Nur 3 Kinder der EG1 und jeweils 1 Kind der EG2 und EG3 waren nach der Geburt ohne Entzugssymptomatik. Die durchschnittliche Entzugssymptomatik zum Zeitpunkt der Geburt war in den Gruppen EG1, EG2 und EG3, wie aus Tabelle 9.6 erkennbar, annähernd gleich groß.

Tab. 9.6 Schwere des Entzugssyndroms

\begin{tabular}{|l|c|c|c|c|c|}
\hline & EG1 $(\mathbf{N}=16)$ & EG2 $(\mathbf{N}=16)$ & EG3 $(\mathbf{N}=13)$ & F & Sig. \\
\hline \multicolumn{2}{|l|}{ Schwere des Entzugs nach Finnegan } \\
\hline MW (SD) & $7,44(4,49)$ & $7,75(5,13)$ & $7,23(4,55)$ & 0,044 & 0,957 \\
\hline
\end{tabular}

Der Anteil der Kinder, deren Mütter zum Zeitpunkt der Geburt substituiert waren, betrug in Gruppe EG1 72,2\%, in Gruppe EG2 21,1\% und in Gruppe EG3 64,3\% (siehe auch Tabelle $8.11)$

\subsection{Korrelationsstatistische Auswertung}

Um die Zusammenhänge zwischen den potentiell konfundierenden Variablen und den Kriterien zu überprüfen sind im Folgenden die Korrelationstabellen für alle Kinder der EG (EG1, EG2 und EG3) sowie für die KG1 erstellt. Die Korrelation der Variablen Psychopathologie und Schulbildung der Mütter mit den Kriterien wird speziell für die Untergruppe der leiblichen Mütter (EG1, KG1) betrachtet, da nur in diesen Gruppen ein kontinuierliches Zusammenleben erfolgt war und nur innerhalb dieser Gruppen Aussagen über genetische Einflüsse möglich sind. 


\subsubsection{Korrelationskoeffizienten für EG1, EG2, EG3}

In Tabelle 9.8 markieren die grau unterlegten Felder die Korrelationen der Kriterien mit den Einflussvariablen.

Tab. 9.8 Korrelationskoeffizienten (r) der Gruppe opiatexponierter Kinder

\begin{tabular}{|c|c|c|c|c|c|c|c|c|c|c|c|c|c|}
\hline & 1 & 2 & 3 & 4 & 5 & 6 & 7 & 8 & 9 & 10 & 11 & 12 & 13 \\
\hline $1 \mathrm{GAF}$ & 1,00 & & & & & & & & & & & & \\
\hline 2 Gesamt-IQ &,$- 463^{\star *}$ & 1,00 & & & & & & & & & & & \\
\hline $3 \mathrm{H}-\mathrm{IQ}$ &,$- 498^{* *}$ &, $853^{* *}$ & 1,00 & & & & & & & & & & \\
\hline 4 V-IQ &,$- 335^{*}$ & $891^{* *}$ &, $533^{* *}$ & 1,00 & & & & & & & & & \\
\hline 5 CBCL Ges. &, $725^{\star *}$ &,$- 304^{*}$ &,- 272 &,- 288 & 1,00 & & & & & & & & \\
\hline 6 CBCL Int. &, $593^{* *}$ &,$- 310^{*}$ &,- 276 &,$- 299^{*}$ &, $850^{* *}$ & 1,00 & & & & & & & \\
\hline 7 CBCL Ext. &, $723^{* *}$ &,$- 300^{*}$ &,- 264 &,$- 292^{*}$ &, $910^{* *}$ &, $673^{* *}$ & 1,00 & & & & & & \\
\hline 8 Alter & ,140 & ,007 & 056 &,- 058 & 201 & ,112 & 0,200 & 1,00 & & & & & \\
\hline 9 Geb.gew. &,- 180 & ,079 &,- 091 & ,087 &,- 138 &,- 069 &,- 025 &,- 219 & 1,00 & & & & \\
\hline 10 Gest.alt. &,- 250 & -137 &, 013 & ,060 &,- 071 & ,003 &,- 137 &,- 205 &, $728^{* *}$ & 1,00 & & & \\
\hline 11 NAS & ,199 &,$- 341^{*}$ &,$- 323^{*}$ &,$- 341^{\star}$ &, 013 &,- 029 & 059 &,- 216 & , 100 & ,076 & 1,00 & & \\
\hline 12 Achse V & '203 &,- 111 &,- 142 &,- 066 & ,355* & ,307* & ,319* & ,314* &,- 009 & ,118 &,- 098 & 1,00 & \\
\hline 13 B-wechsel ${ }^{1}$ & ,239 & ,071 & ,078 & ,115 & 270 & ,140 &, $327^{*}$ & ,280* &,- 171 &,- 123 &,- 031 &, $705^{* *}$ & 1,00 \\
\hline
\end{tabular}

* Signifikanzniveau $<0,05$

** Signifikanzniveau $<0,01$

${ }^{1}$ Anzahl der Betreuungswechsel

Innerhalb der unabhängigen Variablen besteht eine Korrelation zwischen Alter und Achse V und Alter und Anzahl der Betreuungswechsel - mit einem höheren Lebensalter steigt erwartungsgemäß auch die bisher erlebte psychosoziale Belastung bzw. die Wahrscheinlichkeit für einen Wechsel der Bezugsperson. Ebenfalls erwartungsgemäß ist der Zusammenhang zwischen den unabhängigen Variablen Geburtsgewicht und Gestationsalter sowie der Achse V und Anzahl der Betreuungswechsel.

Positive Zusammenhänge zwischen Einflussvariablen und Kriterien bestehen zwischen der Ausprägung der Belastung auf der Achse V und der Psychopathologie der Kinder in allen drei CBCL-Skalen. Speziell die Skala Externalisierend der CBCL ist positiv korreliert mit der Anzahl der erlebten Betreuungswechsel. Zwischen dem Kriterium GAF und den unabhängigen Variablen bestehen keinerlei Zusammenhänge. Keinerlei Zusammenhänge finden sich auch zwischen den Kriterien und den unabhängigen Variablen Alter, Geburtsgewicht und Gestationsalter. 


\subsubsection{Zusammenhang NAS und kognitive Entwicklung (Hypothese II)}

Zwischen der Schwere des NAS und den Gesamtskalen des HAWIK-III besteht wie aus Tabelle 9.8 ersichtlich ein statistisch signifikanter negativer Zusammenhang (Gesamt-IQ: $\mathrm{p}=$ 0,025; Handlungs-IQ: $p=0,039$; Verbal-IQ: $p=0,029$ ), womit Hypothese II bestätigt ist. Je ausgeprägter die Entzugssymptomatik, desto niedriger ist die kognitive Leistung. Der Zusammenhang ist in Abbildung 9.1 graphisch für den Gesamt-IQ dargestellt. Zur besseren Veranschaulichung wurden sowohl Gesamt-IQ als auch die Entzugssymptomatik kategorisiert und auch in Form einer Tabelle (Tab. 9.10) dargestellt. Ebenfalls wurde der durchschnittliche Gesamt-IQ für die Kategorien der unterschiedlichen Schwere der Entzugssymptomatik dargestellt.

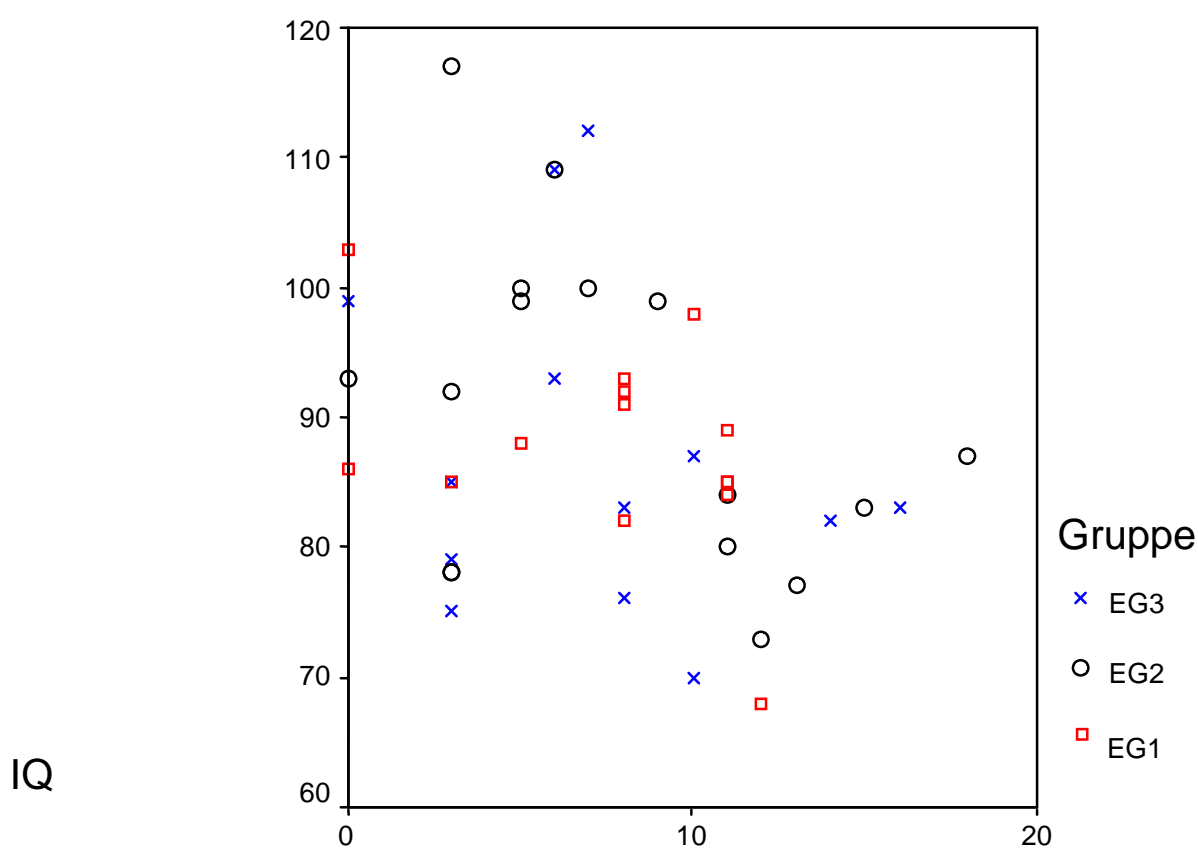

Finnegan Summenscore

Abb. 9.1 Histogramm IQ/Schwere des NAS in Abhängigkeit der Gruppe

Die Grafik und die nachfolgende Tabelle 9.10 illustrieren den negativen Zusammenhang: Bei fehlender Entzugssymptomatik ist die kognitive Leistung durchgängig im Durchschnittsbereich, bei schwer ausgeprägter Symptomatik ist sie vorwiegend unterdurchschnittlich ausgeprägt. Weniger eindeutig ist der Zusammenhang im Bereich des leichten und mittelschweren NAS und die kognitive Leistung in der Gruppe des 
mittelschweren Abstinenzsyndroms ist besser als in der Gruppe der leicht ausgeprägten Entzugssymptomatik.

Tab. 9.10 Verteilung IQ und Schwere des NAS

\begin{tabular}{|c|c|c|c|c|}
\hline & Kein NAS & Leichtes NAS & Mittelschweres NAS & Schweres NAS \\
\hline IQ $<70$ & - & - & - & $1(6,3 \%)$ \\
\hline $70-84$ & - & $4(36,4 \%)$ & $3(25 \%)$ & $956,3 \%)$ \\
\hline $85-100$ & $3(75 \%)$ & $6(54,5 \%)$ & $6(50 \%)$ & $6(37,5 \%)$ \\
\hline $101-115$ & $1(25 \%)$ & - & $3(25 \%)$ & - \\
\hline $116-130$ & - & $1(9,1 \%)$ & - & - \\
\hline MW (SD) & $95,25(7,41)$ & $88,73(12,59)$ & $94,92(11,4)$ & $82,19(7,45)$ \\
\hline
\end{tabular}

Anmerkung: Ein leichter Entzug bedeutet 1-5 Punkte; ein mittelschwerer Entzug 6-9 Punkte, ein schwerer Entzug bedeutete $>=10$ Punkte auf der Skala nach Finnegan.

\section{Substitution, Schwere des NAS und IQ}

Da die Substitution auch mit einer stärkeren Entzugssymptomatik einhergeht, interessierte noch die Verteilung von Intelligenz und Schwere des NAS in Abhängigkeit vom Faktor Substitution. Die Abbildung 9.2 stellt diesen Zusammenhang graphisch dar, Tabelle 9.11 vergleicht den IQ kategorisiert und gemittelt sowie die Schwere des NAS in Abhängigkeit des Faktors Substitution.

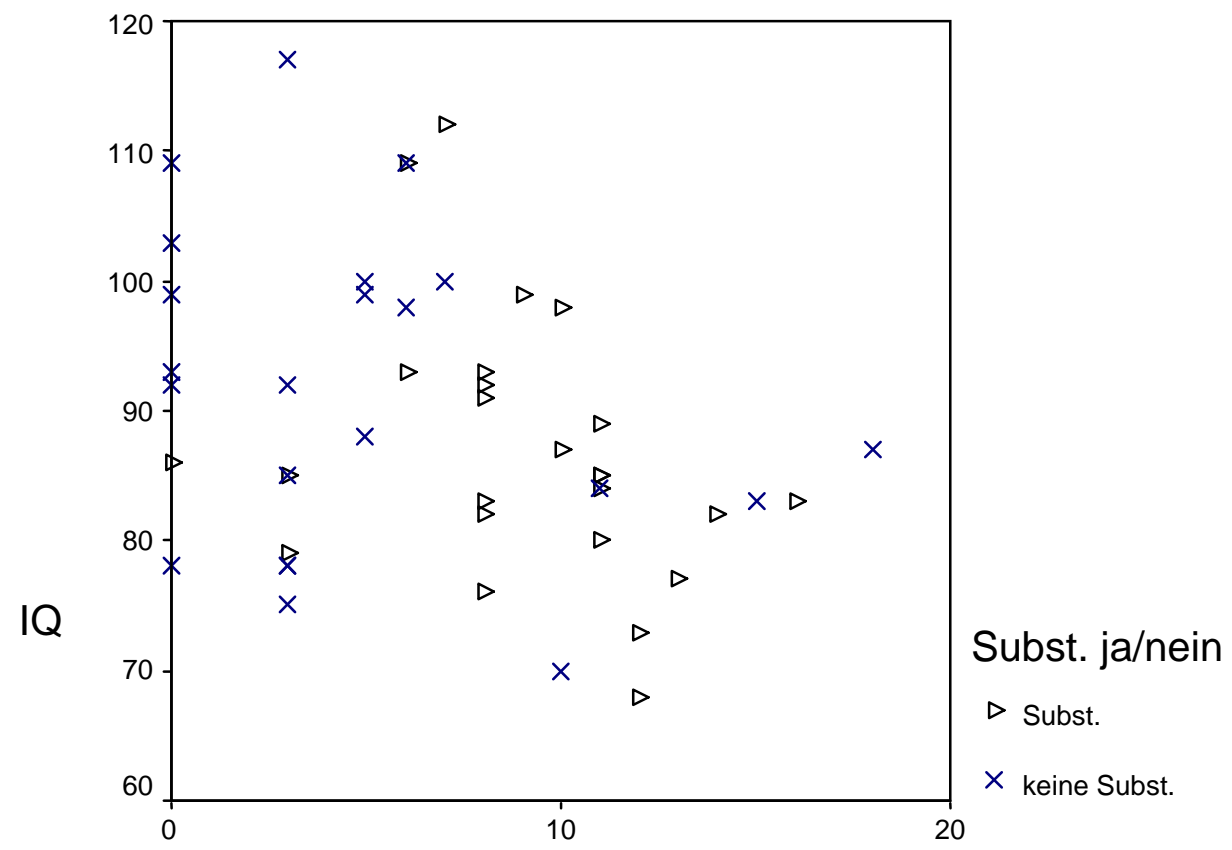

Finnegan Summenscore

Abb. 9.2 Histogramm IQ/Schwere des NAS in Abhängigkeit des Faktors Substitution 
Tab. 9.11 Verteilung IQ und Schwere des NAS in Abhängigkeit vom Faktor Substitution

\begin{tabular}{|c|c|c|}
\hline IQ & Substitution $(\mathbf{N}=\mathbf{2 6})$ & Keine Substitution $(\mathbf{N}=\mathbf{2 1})$ \\
\hline IQ $<70$ & - & $1(3,8 \%)$ \\
\hline $70-84$ & $8(38,1 \%)$ & $10(38,5 \%)$ \\
\hline $85-100$ & $9(42,9 \%)$ & $13(50 \%)$ \\
\hline $101-115$ & $3(14,3 \%)$ & - \\
\hline $116-130$ & $1(4,8 \%)$ & $90,81(12,24)$ \\
\hline MW (SD) & $86,88(9,97)$ & \\
\hline Schwere des Entzugssyndroms & & $5,65(5,20)$ \\
\hline MW (SD) & $8,96(3,60)$ & \\
\hline
\end{tabular}

Anmerkung: Ein leichter Entzug bedeutet 1-5 Punkte; ein mittelschwerer Entzug 6-9 Punkte, ein schwerer Entzug bedeutete $>=10$ Punkte auf der Skala nach Finnegan.

Ein Gruppenvergleich mittels t-Test zeigt, dass es beim Gesamt-IQ keine signifikanten Unterschiede zwischen Kindern substituierter und nicht-substituierter Mütter gibt $(t=1,212$, $\mathrm{df}=45, \mathrm{p}=0,232)$.

Zwischen durchschnittlicher Schwere des Entzugssyndroms von Kindern substituierter vs. nicht substituierter Mütter besteht hingegen ein signifikanter Unterschied, $\mathrm{t}=-2,518, \mathrm{df}=45$, $\mathrm{p}=0,016$.

\subsubsection{Korrelationskoeffizienten für KG1}

In Tabelle 9.12 sind die Korrelationskoeffizienten für die KG1 aufgelistet, grau unterlegte Felder markieren wieder die Korrelationen der Kriterien mit den Einflussvariablen.

Tab. 9.12 Korrelationskoeffizienten der Kinder der Kontrollgruppe

\begin{tabular}{|l|l|l|l|l|l|l|l|l|l|l|l|}
\hline & 1 & 2 & 3 & 4 & 5 & 6 & 7 & 8 & 9 & 10 & 11 \\
\hline 1 GAF & 1,00 & & & & & & & & & & \\
\hline 2 Gesamt-IQ &,- 197 & 1,00 & & & & & & & & & \\
\hline 3 H-IQ &,- 206 &, $927^{* *}$ & 1,00 & & & & & & & & \\
\hline 4 V-IQ &,- 238 &, $945^{* *}$ &, $779^{* *}$ & 1,00 & & & & & & & \\
\hline 5 CBCL Ges. &, $577^{*}$ &,$- 161^{*}$ &,- 108 &,- 250 & 1,00 & & & & & & \\
\hline 6 CBCL Int. &, 508 &,- 237 &,- 189 &,- 306 &, $855^{* *}$ & 1,00 & & & & & \\
\hline 7 CBCL Ext. &, 203 &,- 032 &, 124 &,- 101 &, $755^{* *}$ &, 466 & 1,00 & & & & \\
\hline 8 Alter &, $783^{* *}$ &, 082 &,- 006 &,- 201 &, 478 &, 296 &, 278 & 1,00 & & & \\
\hline 9 Geb.gew. &,- 427 &, 223 &, 259 &, 175 &,- 108 &,- 140 &,- 205 &,- 165 & 1,00 & & \\
\hline 10 Gest.alt. &,- 424 &, 340 &, 313 &, 263 &, 291 &, 281 &,- 042 &,- 070 &, $861^{* *}$ & 1,00 & \\
\hline 11 Achse V &, $601^{*}$ &,- 220 &,- 189 &,- 291 &, $612^{*}$ &, $540^{*}$ &, 204 &, $744^{* *}$ &, 121 &, 327 & 1,00 \\
\hline
\end{tabular}

* Signifikanzniveau $<0,05$

** Signifikanzniveau $<0,01$ 
Auch innerhalb der KG1 bestehen wie bei den Kindern mit Opiatexposition zwischen den unabhängigen Variablen Alter und Achse V sowie Geburtsgewicht und Gestationsalter signifikante Zusammenhänge.

Ein statistisch signifikanter positiver Zusammenhang besteht zwischen dem Alter und dem Ausprägungsgrad der Achse VI, der GAF. Die Achse V korreliert positiv mit den CBCLSkalen „Gesamt“ und „Internalisierend“.

\subsubsection{Korrelationen für mütterliche Psychopathologie und Schulbildung}

Tabelle 9.13 zeigt die Korrelationskoeffizienten speziell für die Untergruppe der leiblichen Mütter (KG1 und EG1) für die Variablen Schulbildung der Mutter und Psychopathologie der Mutter. Die Darstellung ist auf die Korrelationen der Kriterien mit den Einflussvariablen beschränkt. Grau unterlegte Felder markieren signifikante Korrelationen.

Tab. 9.13 Korrelationskoeffizienten für die Gruppen EG1 und KG1

\begin{tabular}{|l|l|l|l|l|l|l|l|}
\hline & GAF & Gesamt-IQ & H-IQ & V-IQ & $\begin{array}{l}\text { CBCL- } \\
\text { Gesamt }\end{array}$ & CBCL Int. & CBCL Ext. \\
\hline 12 ASR Mutter &, 355 &,- 077 &,- 077 &,- 024 & $\mathbf{5 3 6}^{\star \star}$ & $\mathbf{, 4 2 1}^{\boldsymbol{*}}$ & $\mathbf{, 4 8 4}^{\text {** }}$ \\
\hline 13 Schule Mutter &, 157 & $\mathbf{4 3 3 1 ^ { \boldsymbol { * } }}$ &, 354 & $\mathbf{, 4 6 6 ^ { * }}$ &, 142 &,- 051 & $\mathbf{4 1 5}^{\boldsymbol{*}}$ \\
\hline
\end{tabular}

* Signifikanzniveau $<0,05$

** Signifikanzniveau $<0,01$

Positive Zusammenhänge finden sich zwischen der Schulbildung der Mutter und den Intelligenztest-Skalen Gesamt-IQ und Verbal-IQ. Der Ausprägungsgrad der Psychopathologie der Mutter korreliert auch positiv mit allen CBCL-Skalen. Paradoxerweise besteht auch ein positiver Zusammenhang zwischen der Schulbildung der Mutter und der externalisierenden Verhaltenssymptomatik, d.h. je höher die Schulbildung der Mutter, desto höher ist auch die Auffälligkeit des Kindes.

\subsubsection{Auswertung für die Hypothesenüberprüfung III und IV}

Zur Beantwortung der Hypothesen III und IV wird zunächst ein Vergleich zwischen allen vier Gruppen durchgeführt. Die Gruppenzugehörigkeit beinhaltet sowohl eine Aussage über das Vorliegen der Opiatexposition als auch über das Ausmaß der psychosozialen Belastung (Hypothese I). Basierend auf diesem Auswertungsdesign und den in den vorhergehenden Abschnitten ermittelten Ergebnissen wird mit den potentiell konfundierenden Variablen in der Kovarianzanalyse folgendermaßen verfahren: 
- Die potentiellen Einflussfaktoren Geburtsgewicht und Gestationsalter sind unspezifische körperliche Risikovariablen, die bei Kindern mit pränataler Opiatexposition verstärkt auftreten und auch Auswirkungen auf die kognitive und sozial-emotionale Entwicklung haben können. In dieser Untersuchung werden diese beiden Variablen als hinreichend kontrolliert betrachtet, da keine statistisch signifikanten Gruppenunterschiede bestehen und da weder innerhalb der EG noch innerhalb der KG1 ein signifikanter Zusammenhang zwischen Gewicht/ Gestationsalter und den Kriterien existiert.

- Bezüglich der Variablen Alter und Geschlecht wurde in der Literatur immer wieder beschrieben, dass sie Gruppenunterschiede beeinflussen und moderieren können. Die vorliegenden Daten zeigen keinen signifikanten Unterschied zwischen den Gruppen, zumindest in Bezug auf das Kriterium GAF findet sich aber ein hoch signifikanter Einfluss des Alters. Da diese beiden Variablen auch vollständig vorliegen, werden sie in die Analyse miteinbezogen.

- Die psychosoziale Belastung ist zweifellos ein sehr relevanter Einflussfaktor für die kindliche Entwicklung. Die entsprechende Variable, der Gesamtscore auf der Achse $\mathrm{V}$, ist durch die Definierung der Gruppeneinteilung (siehe Hypothese I) aber schon im Faktor Gruppenzugehörigkeit enthalten, so dass diese Variable trotz (definitionsgemäßer) signifikanter Unterschiede in den einzelnen Gruppen und trotz signifikant positiver Korrelationen zwischen der Achse V und dem Kriterium CBCL in den Gruppenvergleichen nicht als Kovariate eingeführt wird. Mit der gleichen Begründung wird auch die Variable Anzahl der Betreuungswechsel nicht in den Gruppenvergleichen berücksichtigt. Aufgrund des hohen Aussagewertes der Achse V wird diese Variable aber als Einflussfaktor in der nachfolgenden Regressionsanalyse definiert.

- Die Einflussvariablen Psychopathologie der Mutter und Schulbildung der Mutter können beim Vergleich der vier Gruppen nicht als Kontrollvariablen berücksichtigt werden, da sich der Effekt dieser Variablen für die Gruppe EG3 auf Grund der teilweise erst sehr kurzen Betreuungszeiten der Bezugspersonen nicht sinnvoll interpretieren lässt. Zusätzlich käme es zu einer Vermischung von einem reinen Umwelteinfluss (Gruppe EG2) und einem Einfluss der sowohl genetisch als auch als Umwelteinfluss wirkt (EG1, KG1). Für potentiell signifikante Gruppenunterschiede zwischen EG1 und KG1 werden aber innerhalb dieser beiden Gruppen zusätzliche 
Kovarianzanalysen unter Einbeziehung der Variabeln Schulbildung und Psychopathologie berechnet.

- Die Einflussvariable NAS bezieht sich nur auf die Gruppe der opiatexponierten Kinder und kann deshalb nicht in den Gesamtgruppenvergleich miteinbezogen werden. Der Einfluss auf die kognitive Entwicklung wurde in Abschnitt 9.2.1.1 genau untersucht. 


\subsection{Kognitive Entwicklung}

Der HAWIK-III konnte bei fast allen Kindern, von denen nicht nur Fragebögen vorlagen, durchgeführt werden. In zwei Fällen (beide Kinder aus EG3) wollte die Mutter die Kinder nicht einer erneuten Leistungsdiagnostik aussetzen, stellte uns aber das kurz zuvor von einer psychiatrischen Ambulanz ermittelte Ergebnis zur Verfügung. Bei einem Kind aus der EG1 $(\mathrm{IQ}=55)$ und einem Kind aus der KG1 (IQ = 42) fiel das Ergebnis des HAWIK III in den Bereich der geistigen Behinderung und wurde bei der Mittelwertsbildung nicht berücksichtigt, um die Ergebnisse nicht zu verzerren. Ein Kind erzielte einen im Grenzbereich der geistigen Behinderung liegenden Gesamt-IQ von 68 (V-IQ 74; H-IQ 65). Dieser Wert stellte allerdings keinen Ausreißer dar, sondern repräsentierte den unteren Bereich der kognitiven Leistung in unserer Probandengruppe. Der Wert wurde deshalb in den weiteren Berechnungen berücksichtigt.

\subsubsection{Gruppeneffekte}

Es wird ein Gruppenvergleich zwischen allen vier Gruppen durchgeführt. Wie in Abschnitt 9.2.4 beschrieben, wurden die Variablen Alter und Geschlecht kontrolliert. Die Ergebnisse der Gesamt- und Subskalen sind aus Tabelle 9.14 ersichtlich. Neben der Prüfgröße F und dem Signifikanzniveau p wird die Effektstärke ?² angegeben.

Tab. 9.14 Intelligenztestergebnisse

\begin{tabular}{|c|c|c|c|c|c|c|}
\hline & $\begin{array}{c}\text { EG1 } \\
(N=16) \\
\end{array}$ & $\begin{array}{c}\text { EG2 } \\
(N=19)\end{array}$ & $\begin{array}{c}\text { EG3 } \\
(\mathrm{N}=14)\end{array}$ & $\begin{array}{c}\text { KG1 } \\
(N=14)\end{array}$ & $\begin{array}{c}\text { EG1 v. EG2 v. } \\
\text { EG3 v. KG1 }\end{array}$ & \\
\hline & MW (SD) & MW (SD) & MW (SD) & MW (SD) & F (p) ? $?^{2}$ & \\
\hline \multicolumn{7}{|l|}{ Gesamtskalen } \\
\hline Verbal-IQ & $\begin{array}{c}93,88 \\
(12,44)\end{array}$ & $\begin{array}{l}93,84 \\
(13,09)\end{array}$ & $\begin{array}{l}95,67 \\
(12,62)\end{array}$ & $\begin{array}{c}97,93 \\
(18,94)\end{array}$ & $0,482(0,696) 0,027$ & \\
\hline Handlungs-IQ & $\begin{array}{l}83,38 \\
(7,81)\end{array}$ & $\begin{array}{l}88,26 \\
(11,17)\end{array}$ & $\begin{array}{c}81,50 \\
(12,76)\end{array}$ & $\begin{array}{c}95,71 \\
(15,42)\end{array}$ & $3,818(0,015) 0,181$ & $E G 1, E G 3<K G 1$ \\
\hline Gesamt-IQ & $\begin{array}{l}87,25 \\
(7,88)\end{array}$ & $\begin{array}{l}90,47 \\
(12,25)\end{array}$ & $\begin{array}{c}86,79 \\
(12,38)\end{array}$ & $\begin{array}{l}96,50 \\
(17,01)\end{array}$ & $2,095(0,112) 0,104$ & \\
\hline \multicolumn{7}{|c|}{ Subskalen (Wertpunkte) } \\
\hline $\begin{array}{l}\text { Allgemeines } \\
\text { Wissen }\end{array}$ & $\begin{array}{l}8,81 \\
(2,40)\end{array}$ & $\begin{array}{l}7,68 \\
(3,22)\end{array}$ & $\begin{array}{c}8,08 \\
(2,39)\end{array}$ & $\begin{array}{l}8,50 \\
(3,18)\end{array}$ & $0,146(0,932) 0,008$ & \\
\hline $\begin{array}{l}\text { Zahlennach- } \\
\text { sprechen* }\end{array}$ & $\begin{array}{l}7,71 \\
(3,04)\end{array}$ & $\begin{array}{l}7,94 \\
(2,38)\end{array}$ & $\begin{array}{l}8,00 \\
(3,95)\end{array}$ & $\begin{array}{l}8,00 \\
(2,58)\end{array}$ & $0,091(0,964) 0,008$ & \\
\hline
\end{tabular}


Tab. 9.14 (Fortsetzung) Intelligenztestergebnisse

\begin{tabular}{|c|c|c|c|c|c|c|}
\hline & $\begin{array}{c}\text { EG1 } \\
(\mathrm{N}=16)\end{array}$ & $\begin{array}{c}\mathrm{EG} 2 \\
(\mathrm{~N}=19)\end{array}$ & $\begin{array}{c}\text { EG3 } \\
(\mathrm{N}=14)\end{array}$ & $\begin{array}{c}\text { KG1 } \\
(\mathrm{N}=14)\end{array}$ & $\begin{array}{c}\text { EG1 v. EG2 v. } \\
\text { EG3 v. KG1 }\end{array}$ & \\
\hline & MW (SD) & MW (SD) & MW (SD) & MW (SD) & $\mathrm{F}(\mathrm{p}) ?^{2}$ & \\
\hline Wortschatz-Test & $\begin{array}{l}8,63 \\
(3,40)\end{array}$ & $\begin{array}{l}9,11 \\
(3,03)\end{array}$ & $\begin{array}{l}9,83 \\
(2,37)\end{array}$ & $\begin{array}{c}8,86 \\
(4,54)\end{array}$ & $0,768(0,517) 0,042$ & \\
\hline $\begin{array}{l}\text { Rechnerisches } \\
\text { Denken }\end{array}$ & $\begin{array}{c}8,88 \\
(3,24)\end{array}$ & $\begin{array}{l}8,32 \\
(2,11)\end{array}$ & $\begin{array}{l}8,75 \\
(2,99)\end{array}$ & $\begin{array}{l}10,36 \\
(4,11)\end{array}$ & $1,283(0,290) 0,069$ & \\
\hline $\begin{array}{l}\text { Allgemeines } \\
\text { Verständnis }\end{array}$ & $\begin{array}{c}9,75 \\
(2,65)\end{array}$ & $\begin{array}{l}9,26 \\
(2,64)\end{array}$ & $\begin{array}{c}8,83 \\
(4,20)\end{array}$ & $\begin{array}{c}9,64 \\
(3,20)\end{array}$ & $0,139(0,936) 0,008$ & \\
\hline $\begin{array}{l}\text { Gemeinsamkeiten } \\
\text { finden }\end{array}$ & $\begin{array}{c}8,75 \\
(3,17)\end{array}$ & $\begin{array}{c}9,47 \\
(2,57)\end{array}$ & $\begin{array}{l}10,33 \\
(1,83)\end{array}$ & $\begin{array}{l}10,00 \\
(4,17)\end{array}$ & $1,307(0,282) 0,070$ & \\
\hline Bilderergänzen & $\begin{array}{l}9,88 \\
(4,79)\end{array}$ & $\begin{array}{l}8,74 \\
(3,11)\end{array}$ & $\begin{array}{c}7,92 \\
(2,27)\end{array}$ & $\begin{array}{l}9,79 \\
(2,39)\end{array}$ & $0,912(0,442) 0,050$ & \\
\hline Bilderordnen & $\begin{array}{l}7,19 \\
(2,71)\end{array}$ & $\begin{array}{l}8,26 \\
(2,92)\end{array}$ & $\begin{array}{c}7,67 \\
(2,99)\end{array}$ & $\begin{array}{c}9,36 \\
(3,69)\end{array}$ & $1,580(0,205) 0,084$ & \\
\hline Mosaiktest & $\begin{array}{c}6,13 \\
(2,66)\end{array}$ & $\begin{array}{l}7,63 \\
(3,09)\end{array}$ & $\begin{array}{l}5,67 \\
(3,73)\end{array}$ & $\begin{array}{l}7,93 \\
(3,65)\end{array}$ & $1,715(0,175) 0,090$ & \\
\hline Figurenlegen & $\begin{array}{c}5,69 \\
(2,21)\end{array}$ & $\begin{array}{c}7,42 \\
(1,84)\end{array}$ & $\begin{array}{l}6,42 \\
(2,39)\end{array}$ & $\begin{array}{c}8,93 \\
(3,12)\end{array}$ & $4,169(0,010) 0,194$ & $\begin{array}{c}\text { EG1, EG3 <KG1 } \\
\text { Trend }(p=0,096) \\
E G 2<K G 1\end{array}$ \\
\hline $\begin{array}{l}\text { Zahlen-Symbol- } \\
\text { Test }\end{array}$ & $\begin{array}{l}7,94 \\
(3,26)\end{array}$ & $\begin{array}{l}8,37 \\
(2,50)\end{array}$ & $\begin{array}{l}6,33 \\
(2,42)\end{array}$ & $\begin{array}{l}10,07 \\
(2,89)\end{array}$ & $3,755(0,016) 0,178$ & $\begin{array}{c}E G 1, E G 3<K G 1 \\
E G 3<E G 2 \\
\text { Trend }(p=0,099) \\
E G 2<K G 1\end{array}$ \\
\hline
\end{tabular}

* Beim Zahlennachsprechen entsprach die Zahl der Kinder pro Gruppe nicht dem oben angegebenen N, sondern betrug: $\mathrm{EG} 1=7, \mathrm{EG} 2=17, \mathrm{EG} 3=11, \mathrm{KG} 1=10$

Bei drei Skalen bestehen signifikante Unterschiede zwischen den Gruppen. Im Handlungsteil erzielen die Kinder der EG3 und der EG1 ein schlechteres Ergebnis als die KG1, zwischen EG2 und KG1 bestehen keine signifikanten Unterschiede. In den zwei Subskalen ZahlenSymbol-Test und Figurenlegen schneiden die EG1 und EG3 ebenfalls signifikant schlechter ab als die KG1. Bei beiden Kriterien besteht ein Trend hinsichtlich eines niedrigeren Ergebnisses der EG2 im Vergleich zur KG1. Beim Zahlen-Symbol-Test besteht auch ein signifikanter Unterschied zwischen EG2 und EG3. Geschlecht und Alter haben in keinem Fall einen signifikanten Einfluss.

\section{Einfluss der mütterlichen Schulbildung}

Da die mütterliche Schulbildung eine hohe Korrelation zum Gesamt- und Verbal-IQ aufwies (siehe Tabelle 9.13), wurde auch eine Kovarianzanalyse für die Gruppe der bei den leiblichen Mütter lebenden Kinder (EG1 und KG1) berechnet. Zusätzlich zu Alter und Geschlecht wurde die Schulbildung der Mutter kontrolliert. Die Gruppenunterschiede zwischen EG1 und KG1 blieben auch nach Kontrolle der Variable mütterliche Schulbildung signifikant, ein 
signifikanter Einfluss der Schulbildung bestand nur bei der Variable Handlungs-IQ ( $\mathrm{p}=$ $0,003)$.

\subsubsection{Verteilung innerhalb der Gruppen}

Zur besseren Veranschaulichung sind die IQ-Verteilungen innerhalb der vier Gruppen tabellarisch dargestellt, und zwar für den Gesamt-IQ (Tabelle 9.15), den Verbal-IQ (Tabelle 9.16) und den Handlungs-IQ (Tabelle 9.17).

\section{Verteilung Gesamt-IQ}

Tabelle 9.15 Verteilung des Gesamt-IQ in den vier Gruppen

\begin{tabular}{|l|c|c|c|c|}
\hline \multicolumn{2}{|c|}{ EG 1 $(\mathbf{N}=\mathbf{1 6})$} & EG 2 $(\mathbf{N}=\mathbf{1 9})$ & EG 3 (N = 14) & KG 1 (N = 14) \\
\hline Hawik-III Gesamt-IQ & \multicolumn{3}{|c|}{-} \\
\hline$<\mathbf{7 0}$ & $1(6,3 \%)$ & - & - & $4(28,6 \%)$ \\
\hline $\mathbf{7 0}-\mathbf{8 4}$ & $3(18,8 \%)$ & $8(42,1 \%)$ & $8(57,1 \%)$ & $5(35,7 \%)$ \\
\hline $\mathbf{8 5}-\mathbf{1 0 0}$ & $11(68,8 \%)$ & $8(42,1 \%)$ & $4(28,6 \%)$ & $4(28,6 \%)$ \\
\hline $\mathbf{1 0 1}-\mathbf{1 1 5}$ & $1(6,3 \%)$ & $2(10,5 \%)$ & $2(14,3 \%)$ & - \\
\hline $\mathbf{1 1 6}-\mathbf{1 3 0}$ & - & $1(5,3 \%)$ & - & $1(7,1 \%)$ \\
\hline$>\mathbf{1 3 0}$ & - & - & - & - \\
\hline
\end{tabular}

\section{Verteilung Verbal-IQ}

Tab. 9.16 Verteilung des Verbal-IQ in den vier Gruppen

\begin{tabular}{|l|c|c|c|c|}
\hline \multicolumn{2}{|c|}{ EG 1 $(\mathbf{N}=\mathbf{1 6})$} & EG 2 $(\mathbf{N}=\mathbf{1 9})$ & EG 3 $(\mathbf{N}=\mathbf{1 4})$ & KG 1 (N = 14) \\
\hline Hawik-III Verbal-IQ & - & - & - & $1(7,1 \%)$ \\
\hline$<\mathbf{7 0}$ & $4(25 \%)$ & $6(31,6 \%)$ & $2(16,7 \%)$ & $2(14,3 \%)$ \\
\hline $\mathbf{7 0}-\mathbf{8 4}$ & $7(43,8 \%)$ & $8(42,1 \%)$ & $6(50 \%)$ & $6(42,9 \%)$ \\
\hline $\mathbf{8 5}-\mathbf{1 0 0}$ & $5(31,3 \%)$ & $4(21,1 \%)$ & $3(25 \%)$ & $3(21,4 \%)$ \\
\hline $\mathbf{1 0 1}-\mathbf{1 1 5}$ & - & $1(5,3 \%)$ & $1(8,3 \%)$ & $1(7,1 \%)$ \\
\hline $\mathbf{1 1 6}-\mathbf{1 3 0}$ & - & - & - & $1(7,1 \%)$ \\
\hline$>\mathbf{1 3 0}$ & & & & \\
\hline
\end{tabular}

\section{Verteilung Handlungs-IQ}

Tab. 9.17 Verteilung des Handlungs-IQ in den vier Gruppen

\begin{tabular}{|c|c|c|c|c|}
\hline & EG $1(N=16)$ & EG $2(N=19)$ & EG $3(N=14)$ & KG $1(N=14)$ \\
\hline \multicolumn{5}{|c|}{ Hawik-III Handlungs-IQ } \\
\hline$<70$ & $1(6,3 \%)$ & - & $2(16,7 \%)$ & - \\
\hline $70-84$ & $9(56,3 \%)$ & $7(36,8 \%)$ & $6(50 \%)$ & $3(21,4 \% 9$ \\
\hline $85-100$ & $6(37,5 \%)$ & $9(47,4 \%)$ & $3(25 \%)$ & $6(42,9 \%)$ \\
\hline $101-115$ & - & $3(15,8 \%)$ & $1(8,3 \%)$ & $4(28,6 \%)$ \\
\hline$>130$ & - & - & - & $1(7,1 \%)$ \\
\hline
\end{tabular}


In allen drei Gruppen zeigt sich die auch schon bei der Analyse der Mittelwerte erkennbare Verschiebung nach links zu einer niedrigeren kognitiven Leistung, besonders ausgeprägt ist dies im Handlungs-IQ bei EG1 und EG3 - die meisten Kinder erzielen Leistungen in der Kategorie IQ 70-84. Beim Gesamt-IQ der EG3 verhält es sich ebenso, während die Verteilung des Verbal-IQ am ehesten normalverteilt erscheint und die meisten Fälle sich in den mittleren Kategorien IQ 85-100 und 101-115 befinden. 


\subsection{Psychopathologie}

Der Fragebogen CBCL lag von 50 Kindern der EG und 17 Kindern der KG vollständig ausgefüllt vor.

\subsubsection{Gruppeneffekte}

Tabelle 9.18 zeigt die Ergebnisse der Varianzanalysen der einzelnen Gruppenvergleiche. Geschlecht und Alter waren kontrolliert.

Tab. 9.18 Child Behavior Checklist EG1, EG2, EG3 und KG1

\begin{tabular}{|c|c|c|c|c|c|c|}
\hline & $\begin{array}{c}\text { EG1 } \\
(N=16)\end{array}$ & $\begin{array}{c}\text { EG2 } \\
(\mathbf{N}=19)\end{array}$ & $\begin{array}{c}\text { EG3 } \\
(\mathrm{N}=14)\end{array}$ & $\begin{array}{c}\text { KG1 } \\
(\mathrm{N}=14)\end{array}$ & $\begin{array}{l}\text { EG1 v. EG2 v. } \\
\text { EG3 v. KG1 }\end{array}$ & \\
\hline & MW (SD) & MW (SD) & MW (SD) & MW (SD) & F (p) ? & \\
\hline \multicolumn{7}{|l|}{ Gesamtskalen } \\
\hline Internalisierend & $\begin{array}{l}56,94 \\
(9,53)\end{array}$ & $\begin{array}{c}49,53 \\
(10,09)\end{array}$ & $\begin{array}{c}55,79 \\
(10,09)\end{array}$ & $\begin{array}{l}49,50 \\
(6,24)\end{array}$ & $3,604(0,019) 0,167$ & $\begin{array}{c}\text { EG1 > EG2, KG1 } \\
\text { Trend }(\mathrm{p}=0,086 / 0,076): \\
\text { EG3 }>\text { EG2, KG1 }\end{array}$ \\
\hline Externalisierend & $\begin{array}{l}54,56 \\
(7,68)\end{array}$ & $\begin{array}{c}50,63 \\
(11,39)\end{array}$ & $\begin{array}{c}59,14 \\
(16,30)\end{array}$ & $\begin{array}{l}46,14 \\
(6,68)\end{array}$ & $3,628(0,019) 0,168$ & $\begin{array}{c}\text { EG1, EG3 > KG1 } \\
\text { Trend }(\mathrm{p}=0,090 / 0,073): \\
\text { EG2 < EG1, EG3 }\end{array}$ \\
\hline Gesamtskala & $\begin{array}{l}57,44 \\
(9,54)\end{array}$ & $\begin{array}{c}51,53 \\
(10,43)\end{array}$ & $\begin{array}{c}59,07 \\
(14,72)\end{array}$ & $\begin{array}{l}46,57 \\
(7,47)\end{array}$ & $4,788(0,005) 0,210$ & $\begin{array}{c}\text { EG1 > EG2, KG1 } \\
\text { EG3 > KG1 } \\
\text { Trend }(\mathrm{p}=0,087): \\
\text { EG3 > EG2 } \\
\end{array}$ \\
\hline \multicolumn{7}{|c|}{ Subskalen (T-Werte) } \\
\hline Sozialer Rückzug & $\begin{array}{l}57,87 \\
(8,57)\end{array}$ & $\begin{array}{l}54,47 \\
(5,22)\end{array}$ & $\begin{array}{c}58,79 \\
(10,10) \\
\end{array}$ & $\begin{array}{l}52,07 \\
(4,21)\end{array}$ & $2,992(0,039) 0,143$ & $\begin{array}{c}\text { EG1, EG3 > KG1 } \\
\text { Trend }(p=0,072): \\
\text { EG1 > EG2 }\end{array}$ \\
\hline $\begin{array}{l}\text { Körperliche } \\
\text { Beschwerden }\end{array}$ & $\begin{array}{l}56,94 \\
(5,67)\end{array}$ & $\begin{array}{l}53,32 \\
(5,09)\end{array}$ & $\begin{array}{l}56,21 \\
(6,58)\end{array}$ & $\begin{array}{l}55,29 \\
(5,86)\end{array}$ & $1,491(0,227) 0,076$ & \\
\hline Angst/Depression & $\begin{array}{l}55,13 \\
(7,09) \\
\end{array}$ & $\begin{array}{l}53,79 \\
(5,90) \\
\end{array}$ & $\begin{array}{l}55,79 \\
(6,67) \\
\end{array}$ & $\begin{array}{l}52,57 \\
(3,55) \\
\end{array}$ & $0,811(0,493) 0,043$ & \\
\hline Soziale Probleme & $\begin{array}{c}61,44 \\
(12,23) \\
\end{array}$ & $\begin{array}{l}54,11 \\
(5,92) \\
\end{array}$ & $\begin{array}{c}61,93 \\
(14,02) \\
\end{array}$ & $\begin{array}{l}51,43 \\
(3,27) \\
\end{array}$ & $3,929(0,013) 0,179$ & EG1, EG3 > KG1, EG2 \\
\hline $\begin{array}{l}\text { Schizoid/ } \\
\text { Zwanghaft }\end{array}$ & $\begin{array}{l}54,00 \\
(3,76)\end{array}$ & $\begin{array}{l}51,89 \\
(4,01)\end{array}$ & $\begin{array}{l}56,21 \\
(8,42)\end{array}$ & $\begin{array}{l}53,50 \\
(6,00)\end{array}$ & $1,450(0,238) 0,075$ & \\
\hline $\begin{array}{l}\text { Aufmerksamkeits- } \\
\text { probleme }\end{array}$ & $\begin{array}{l}60,81 \\
(9,57)\end{array}$ & $\begin{array}{l}55,79 \\
(6,62)\end{array}$ & $\begin{array}{c}64,86 \\
(11,20)\end{array}$ & $\begin{array}{l}52,00 \\
(3,14)\end{array}$ & $6,611(0,001) 0,269$ & $\begin{array}{c}\text { EG1, EG3 > KG1 } \\
\text { EG3 > EG2 } \\
\text { Trend }(p=0,06 / 0,052) \\
\text { EG1>EG2; EG2 > KG1 } \\
\end{array}$ \\
\hline $\begin{array}{l}\text { Dissoziales } \\
\text { Verhalten }\end{array}$ & $\begin{array}{l}59,63 \\
(9,41)\end{array}$ & $\begin{array}{l}55,79 \\
(7,26)\end{array}$ & $\begin{array}{c}63,14 \\
(13,01)\end{array}$ & $\begin{array}{l}53,93 \\
(5,88)\end{array}$ & $3,163(0,032) 0,149$ & $\begin{array}{c}\text { EG3 > EG2, KG1 } \\
\text { Trend }(\mathrm{p}=0,051) \\
\text { EG1 }>\text { KG1 }\end{array}$ \\
\hline $\begin{array}{l}\text { Aggressives } \\
\text { Verhalten }\end{array}$ & $\begin{array}{l}55,44 \\
(8,05) \\
\end{array}$ & $\begin{array}{l}54,42 \\
(6,47) \\
\end{array}$ & $\begin{array}{c}61,43 \\
(13,62) \\
\end{array}$ & $\begin{array}{l}50,71 \\
(1,64)\end{array}$ & $3,685(0,017) 0,170$ & $\begin{array}{c}\text { EG1, EG2 > KG1 } \\
\text { EG3 > EG2 } \\
\text { EG3 > KG1 }\end{array}$ \\
\hline
\end{tabular}


In insgesamt acht Skalen, den drei Gesamtskalen und fünf von acht Subskalen, finden sich signifikante Unterschiede. Als durchgängigstes Ergebnis zeigt sich dabei eine signifikant stärkere Beeinträchtigung der EG3 im Vergleich zur KG1 (in sieben dieser acht Skalen, in der Skala Internalisierend besteht diesbezüglich nur ein Trend). In nur zwei der Skalen besteht hingegen ein signifikanter Unterschied (bzw. ein Trend mit p =0,052) zwischen EG2 und KG1, und zwar in den Skalen Aufmerksamkeitsprobleme und Aggressives Verhalten, in denen die EG2 eine höhere Belastung aufweist als die KG1.

Betrachtet man den Einfluss der Variablen Alter und Geschlecht so hat das Geschlecht in den Skalen Sozialer Rückzug und Internalisierend einen signifikanten Einfluss ( $p=0,045$ und $0,025)$ und das Alter in der Skala Gesamt $(\mathrm{p}=0,018)$. Es bestehen keine signifikanten Wechselwirkungen. Die Mittelwerte der männlichen Gruppenmitglieder gegenüber den Werten der Mädchen sind sowohl in der Skala Sozialer Rückzug (MW: EG1 60,67 vs. 56,20; EG2 57,86 vs. 52,50; EG3 62 vs. 56,38; KG1 51,83 vs. 52,25) als auch in der Skala Internalisierend (MW: EG1 61,33 vs. 54,30; EG2 56,29 vs. 45,58; EG3 56,17 vs. 55,50; KG1 50,17 vs. 49,00) vor allem in den Gruppen EG1, EG2 und EG3 höher ausgeprägt.

\section{Einfluss der mütterlichen Psychopathologie}

Da der Gesamtscore im ASR eine hohe Korrelation zu den Psychopathologieskalen der CBCL (siehe Tabelle 9.13) aufweist, wurde, um den Beitrag der mütterlichen Psychopathologie besser einschätzen zu können, auch eine Kovarianzanalyse für die Gruppe der bei den leiblichen Müttern lebenden Kinder (EG1 und KG1) berechnet. Zusätzlich zu Alter und Geschlecht wurde die Psychopathologie der Mutter kontrolliert. In zwei der sieben Skalen in denen signifikante Unterschiede bestanden, Sozialer Rückzug und Aggressives Verhalten, konnte nach Kontrolle dieser Variablen kein signifikanter Gruppeneffekt mehr festgestellt werden, die mütterliche Psychopathologie trägt damit zu den Gruppenunterschieden bei. Es bestand aber weiterhin ein Trend ( $p=0,07$ bzw. $p=0,072)$. Ein signifikanter Einfluss der mütterlichen Psychopathologie zeigte sich nur in der Skala CBCL Gesamt $(p=0,035)$.

\subsubsection{Verteilung der klinisch auffälligen Werte}

Da die Streuung der CBCL-Werte vor allem in der EG beträchtlich ist, wurde auch untersucht, wie viele der Kinder in den Gruppen tatsächlich klinisch relevante T-Werte > 65 aufweisen. Tabelle 9.19 zeigt, dass nur ein einziges Kind in der KG1 einen solchen Wert 
aufweist, während z.B. bei den Aufmerksamkeitsproblemen bis zu 57\% der Kinder der EG3 einen T-Wert > 65 erreichen. In der EG3 weisen fast durchgängig mehr Kinder eine Ausprägung T-Wert > 65 als in der EG1 und EG2. Die einzigen Ausnahmen bilden die Skalen Angst/Depression und Internalisierend.

Tab. 9.19 Anzahl der Kinder mit CBCL-Werten > 65

\begin{tabular}{|l|c|c|c|c|}
\hline CBCL-Skalen & $\begin{array}{c}\text { EG 1 } \\
(\mathbf{N = 1 6})\end{array}$ & $\begin{array}{c}\text { EG 2 } \\
(\mathbf{N}=\mathbf{1 9})\end{array}$ & $\begin{array}{c}\text { EG 3 } \\
(\mathbf{N}=\mathbf{1 4})\end{array}$ & $\begin{array}{c}\text { KG 1 } \\
(\mathbf{N}=\mathbf{1 4})\end{array}$ \\
\hline Sozialer Rückzug & $3(19 \%)$ & $1(5 \%)$ & $3(21 \%)$ & 0 \\
\hline Körperliche Beschwerden & $2(12,5 \%)$ & $1(5 \%)$ & $1(7 \%)$ & 0 \\
\hline Angst/Depression & $2(12,5 \%)$ & $1(5 \%)$ & $2(14 \%)$ & 0 \\
\hline Soziale Probleme & $4(25 \%)$ & $2(11 \%)$ & $5(36 \%)$ & 0 \\
\hline Schizoid/Zwanghaft & 0 & 0 & $2(14 \%)$ & 0 \\
\hline Aufmerksamkeitsprobleme & $5(31 \%)$ & $3(16 \%)$ & $8(57 \%)$ & 0 \\
\hline Dissoziales Verhalten & $5(31 \%)$ & $2(11 \%)$ & $6(43 \%)$ & $1(7 \%)$ \\
\hline Aggressives Verhalten & $2(12,5 \%)$ & $2(11 \%)$ & $6(43 \%)$ & 0 \\
\hline Internalisierend & $4(25 \%)$ & $1(5 \%)$ & $3(21 \%)$ & 0 \\
\hline Externalisierend & $2(12,5 \%)$ & $3(16 \%)$ & $6(43 \%)$ & 0 \\
\hline Gesamt & $5(31 \%)$ & $3(16 \%)$ & $5(36 \%)$ & 0 \\
\hline
\end{tabular}

\subsubsection{ICD 10 Diagnosen}

Zusätzlich zur CBCL wurde das Kiddie-SADS durchgeführt. Bei einem großen Teil der Kinder ist bereits im Vorfeld eine Konsultation wegen psychischer Auffälligkeiten erfolgt. Die Kinder wurden in mindestens einer der folgenden Institutionen vorgestellt: Kinder- und Jugendpsychiatrische Ambulanz, niedergelassener Kinder- und Jugendpsychiater oder Psychologe, Sozialpädiatrisches Zentrum, Erziehungsberatungsstellen, Kinderklinik. Aus Tabelle 9.20 ist ersichtlich, dass die Kinder der Gruppe EG3 mit 79\% die höchste Rate an vorangegangenen Vorstellungen aufweisen und auch in der EG2 liegt diese Zahl mit 58\% der Kinder relativ hoch. Die leiblichen Eltern nahmen mit Abstand am seltensten (24\%) professionelle Beratung in Anspruch. Dementsprechend verhält es sich auch mit den bereits an anderen Institutionen gestellten Diagnosen (Vordiagnose).

Anders sieht es hingegen bei den in unserer Untersuchung vergebenen aktuellen Diagnosen aus: Die Hälfte der Kinder mit späten bzw. häufigen Betreuungswechseln erfüllt aktuell die ICD-10-Kriterien für eine psychiatrische Erkrankung. Ca. 10\% weniger sind es in der Gruppe der Kinder mit leiblichen Eltern und in der Gruppe mit frühem Betreuungswechsel. 
Tab. 9.20 Frühere Konsultationen und ICD-10 Diagnosen

\begin{tabular}{|c|c|c|c|c|}
\hline Anzahl der Kinder mit Diagnosen & $\begin{array}{c}\text { EG } 1 \\
(\mathbf{N}=\mathbf{1 7})\end{array}$ & $\begin{array}{c}\text { EG 2 } \\
(\mathbf{N}=\mathbf{1 9})\end{array}$ & $\begin{array}{c}\text { EG 3 } \\
(N=14)\end{array}$ & $\begin{array}{c}\text { KG 1 } \\
(\mathrm{N}=\mathbf{1 7})\end{array}$ \\
\hline Konsultation im Vorfeld & $4(24 \%)$ & $11(58 \%)$ & $11(79 \%)$ & $3(18 \%)$ \\
\hline Vordiagnose & $1(6 \%)$ & $5(26 \%)$ & $5(35 \%)$ & $1(6 \%)$ \\
\hline Aktuelle Diagnose & $7(41 \%)$ & $7(37 \%)$ & $7(50 \%)$ & $3(18 \%)$ \\
\hline \multicolumn{5}{|l|}{ ICD-10 Diagnose } \\
\hline Verdacht auf tiefgreifende Entwicklungsst. F84.x & & 1 & & \\
\hline Hyperkinetische Störung F90.x & 4 & 4 & 3 & \\
\hline Störung des Sozialverhaltens F91.x & 1 & & 2 & \\
\hline SSV und der Emotionen F92.x & & 1 & 1 & \\
\hline Emotionale Störung F93.x & 1 & & & 1 \\
\hline \multicolumn{5}{|l|}{ Enkopresis F98.1 } \\
\hline Enuresis F98.0 & 1 & 1 & & \\
\hline Bindungsstörung F94.1 & & & 1 & \\
\hline Mittelgradige Intelligenzminderung F71 & & & & 1 \\
\hline Soziale Phobie 40.10 & & & & 1 \\
\hline
\end{tabular}

\subsubsection{Missbrauch psychotroper Substanzen}

Bezüglich einer potentiellen Suchtsymptomatik der Jugendlichen zeigte sich folgendes: Bei insgesamt 3 Jugendlichen konnte ein deutlich erhöhter Zigaretten- und Alkoholkonsum festgestellt werden, die klinischen Kriterien für ein Abhängigkeitssyndrom waren aber nicht erfüllt. Es handelte sich um zwei Jugendliche (ein Mädchen, ein Junge) der Gruppe EG3 und einen Jugendlichen der EG2. Das Alter betrug zwischen 13 Jahren und zwei Monaten und 14 Jahren und 8 Monaten. 


\subsection{Globales psychosoziales Funktionsniveau}

Die Einschätzung der GAF, der Beurteilung des psychosozialen Funktionsniveaus lag von insgesamt 49 Kindern der EG und 15 Kindern der KG vor.

\subsubsection{Gruppeneffekte}

Tabelle 9.21 zeigt die Ergebnisse des Gruppenvergleichs. Je höher der Wert, desto höher ist die durchschnittliche soziale Beeinträchtigung (Scoringschema der Achse VI befindet sich im Anhang). Geschlecht und Alter waren wie in den vorangegangenen Analysen kontrolliert.

Tab. 9.21 GAF-Werte

\begin{tabular}{|l|c|c|c|c|c|}
\hline & EG1 & EG2 & EG3 & KG1 & \multirow{2}{*}{ EG1 v. EG2 v. } \\
& $(\mathbf{N}=\mathbf{1 7})$ & $\mathbf{( N = 1 9 )}$ & $(\mathbf{N}=\mathbf{1 3})$ & $\mathbf{( N = 1 5 )}$ & EG3 v. KG1 \\
\hline & MW (SD) & MW (SD) & MW (SD) & MW (SD) & F (p) $\boldsymbol{?}^{\mathbf{2}}$ \\
\hline GAF & $1,47(1,18)$ & $1,26(1,37)$ & $2,15(1,14)$ & $1,00(1,36)$ & $2,497(0,069) 0,120$ \\
\hline
\end{tabular}

Es besteht ein Trend hinsichtlich einer signifikant höheren Belastung der EG3 auf der Achse VI. Alter $(p=0,005)$ und Geschlecht $(p=0,042)$ haben einen signifikanten Einfluss. Die Mittelwerte der männlichen Gruppenmitglieder sind gegenüber den Werten der Mädchen mit Ausnahme der Gruppe EG3 erhöht (MW: EG1 2,14 vs. 1,00; EG2 1,86 vs. 0,92; EG3 2,00 vs. 2,29; KG1 1,29 vs. 0,75).

\subsubsection{Verteilung der GAF-Werte}

In Tabelle 9.22 ist zur besseren Veranschaulichung die Verteilung der einzelnen GAF-Werte dargestellt.

Tab. 9.22 Verteilung der GAF-Werte

\begin{tabular}{|c|c|c|c|c|}
\hline GAF & EG 1 (N = 17) & EG 2 (N = 19) & EG 3 (N = 13) & KG 1 (N = 15) \\
\hline 0 & $3(17,6 \%)$ & $7(36,8 \%)$ & $1(7,7 \%)$ & $7(46,7 \%)$ \\
\hline 1 & $8(47,1 \%)$ & $6(31,6 \%)$ & $2(15,4 \%)$ & $4(26,7 \%)$ \\
\hline 2 & $2(11,8 \%)$ & $2(10,5 \%)$ & $6(46,2 \%)$ & $3(20 \%)$ \\
\hline 3 & $3(17,6 \%)$ & $2(10,5 \%)$ & $2(15,4 \%)$ & - \\
\hline 4 & $1(5,9 \%)$ & $2(10,5 \%)$ & $2(15,4 \%)$ & - \\
\hline 5 & - & - & - & $1(6,7 \%)$ \\
\hline
\end{tabular}


In der EG2 und KG1 finden sich die höchsten Raten einer herausragenden sozialen Anpassung (0). Betrachtet man die Stufen 0 und 1 (mäßige soziale Funktion) gemeinsam, so fällt die Gruppe EG3 mit insgesamt nur 23,1\% in diesen Kategorien gegenüber den Gruppen EG1 $(64,7 \%)$, EG2 $(68,4 \%)$ und KG1 $(73,4 \%)$ deutlich ab. 


\subsection{Regressionsanalyse}

Abschließend werden die korrelativen Zusammenhänge zwischen den Risikofaktoren biologische Vulnerabilität und psychosoziale Belastung auf die kindliche Entwicklung untersucht (Tabelle 9.23).

Es werden multiple schrittweise Regressionsanalysen gerechnet. Als Kriterien werden die Hauptskalen der drei Bereiche kognitive Entwicklung (HAWIK-III), Psychopathologie (CBCL) und pychosoziale Anpassung (Achse VI) definiert. Es werden verwendet: HAWIK Gesamt-IQ, Verbal-IQ, Handlungs-IQ, die CBCL-Skalen Gesamt, Internalisierend, Externalisierend und die GAF. Als Prädiktoren gehen die psychosoziale Belastung (Ausprägung der Achse V) und die biologischen Vulnerabilität (Drogenexposition ja/nein) in die Analyse ein. Alter und Geschlecht werden ebenfalls als Einflussvariablen definiert.

Tab. 9.23 Regressionsanalyse zur Vorhersage der kindlichen Entwicklung

\begin{tabular}{|c|c|c|c|c|}
\hline & B & SE B & B & $t(p)$ \\
\hline \multicolumn{5}{|l|}{ HAWIK-Gesamt-IQ } \\
\hline $\begin{array}{l}\text { Drogenexposition } \\
\text { Multiple } R=0,257 ; \mathrm{R}^{2}=0,07 \\
\mathrm{~F}(1,64)=4,54 ; \mathrm{p}=0,037\end{array}$ & $-7,515$ & 3,526 & $-0,257$ & $-2,131(0,037)$ \\
\hline \multicolumn{5}{|l|}{ HAWIK-Handlungs-IQ } \\
\hline $\begin{array}{l}\text { Drogenexposition } \\
\text { Multiple } \mathrm{R}=0,341 ; \mathrm{R}^{2}=0,12 \\
\mathrm{~F}(1,62)=8,138 ; \mathrm{p}=0,006\end{array}$ & $-9,775$ & 3,426 & $-0,341$ & $-2,853(0,006)$ \\
\hline \multicolumn{5}{|l|}{ CBCL Gesamt Score } \\
\hline $\begin{array}{l}\text { Achse V } \\
\text { Drogenexposition } \\
\text { Multiple } R=0,492 ; R^{2}=0,24 \\
F(2,63)=10,045 ; p=0,000\end{array}$ & $\begin{array}{l}0,856 \\
6,585\end{array}$ & $\begin{array}{l}0,281 \\
3,017\end{array}$ & $\begin{array}{l}0,351 \\
0,252\end{array}$ & $\begin{array}{l}3,044(0,003) \\
2,183(0,033)\end{array}$ \\
\hline \multicolumn{5}{|l|}{ CBCL Externalisierend } \\
\hline $\begin{array}{l}\text { Achse V } \\
\text { Drogenexposition } \\
\text { Multiple R = 0,408; } \mathrm{R}^{2}=0,17 \\
\mathrm{~F}(2,63)=6,298 ; \mathrm{p}=0,003 \\
\end{array}$ & $\begin{array}{l}0,639 \\
6,570\end{array}$ & $\begin{array}{l}0,300 \\
3,223\end{array}$ & $\begin{array}{l}0,258 \\
0,247\end{array}$ & $\begin{array}{l}2,129(0,037) \\
2,038(0,046)\end{array}$ \\
\hline \multicolumn{5}{|l|}{ CBCL Internalisierend } \\
\hline $\begin{array}{l}\text { Achse V } \\
\text { Geschlecht des Kindes } \\
\text { Multiple } R=0,425 ; \mathrm{R}^{2}=0,18 \\
\mathrm{~F}(2,63)=6,934 ; \mathrm{p}=0,002\end{array}$ & $\begin{array}{c}0,626 \\
-5,629\end{array}$ & $\begin{array}{l}0,239 \\
2,316\end{array}$ & $\begin{array}{c}0,299 \\
-0,278\end{array}$ & $\begin{array}{c}2,614(0,011) \\
-2,431(0,018)\end{array}$ \\
\hline \multicolumn{5}{|l|}{$\boldsymbol{G A F}$} \\
\hline $\begin{array}{l}\text { Achse V } \\
\text { Geschlecht des Kindes } \\
\text { Multiple } R=0,406 ; \mathrm{R}^{2}=0,17 \\
\mathrm{~F}(2,64)=6,316 ; \mathrm{p}=0,003\end{array}$ & $\begin{array}{c}0,087 \\
-0,607\end{array}$ & $\begin{array}{l}0,032 \\
0,300\end{array}$ & $\begin{array}{c}0,317 \\
-0,232\end{array}$ & $\begin{array}{c}2,772(0,007) \\
-2,025(0,047)\end{array}$ \\
\hline
\end{tabular}


Zur Vorhersage des Gesamt-IQ und des Handlungs-IQ erweist sich die Drogenexposition mit einer Varianzaufklärung von 7\% bzw. 12 \% als relevantester Prädiktor. Die Drogenexposition ist assoziiert mit einem geringeren Gesamt- und Handlungs-IQ. Beim Verbal-IQ trägt keiner der Prädiktoren signifikant zur Vorhersage bei.

Drogenexposition und eine hohe psychosoziale Belastung sind assoziiert mit einer hohen Ausprägung der CBCL Gesamtskala und der Skala Externalisierend, die beiden Variablen tragen mit 24\% bzw. 17\% zur Varianzaufklärung bei.

Eine hohe Ausprägung auf der Skala Internalisierend und beim GAF lassen sich am besten durch eine hohe Ausprägung auf der Achse V und durch das männliche Geschlecht vorhersagen, die Varianzaufklärung beträgt $18 \%$ bzw. $17 \%$. 


\section{Diskussion}

Diese Studie hatte zum Ziel, die kognitive und sozial-emotionale Entwicklung pränatal opiatexponierter Kinder zu untersuchen. Der Ausgangsdatensatz, aus dem die Rekrutierung der hier verwendeten Stichprobe erfolgte, wurde retrospektiv aus den Jahren 1988-1995 erhoben. Die hier vorgestellte Untersuchung wurde in den Jahren 2000 - 2003 durchgeführt; zum Zeitpunkt der Untersuchung waren die Kinder mindestens 6 Jahre alt, das älteste untersuchte Kind war 15 Jahre alt, und damit deutlich älter als die untersuchten Kinder in einem Großteil anderer Studien zur Auswirkung pränataler Drogenexposition.

Kinder suchtkranker Eltern haben ein hohes Risiko, Beziehungsabbrüche bzw. einen Wechsel der Hauptbezugsperson zu erleben, und auch in der hier untersuchten Stichprobe zeigte sich bald, dass die Rate an Fremdbetreuungen sehr hoch ist. Es wurde deutlich, dass bei einer Nachuntersuchung dieser Kinder der Einfluss der postnatalen Erziehungsbedingungen stark im Fokus stehen musste, da psychosoziale Einflüsse im Allgemeinen und postnatale Erziehungsbedingungen im Besonderen einen der wesentlichen Bestimmungsfaktoren für die weitere Entwicklung der Kinder substanzabhängiger Eltern darstellen (Ornoy et al., 1996; Brown et al., 2004, Phelps et al., 1997).

Die hohe Zahl der nicht mehr bei den leiblichen Eltern lebenden Kinder barg einerseits die Chance in sich, die Bedeutung unterschiedlicher Betreuungsverhältnisse auf die kindliche Entwicklung innerhalb einer Hochrisikopopulation zu untersuchen, trug aber auch wesentlich zur Komplexität der Untersuchung bei. Dies war einerseits dadurch bedingt, dass erhobene Einflussfaktoren teils von den leiblichen Eltern und teils von nichtverwandten Bezugspersonen stammten und diese Einflüsse teilweise schon das ganze Leben, bei einem kürzlich vorangegangenen Bezugspersonenwechsel aber teilweise auch erst seit kurzer Zeit auf das Kind einwirkten, was das methodische Vorgehen und die Interpretation der Ergebnisse zusätzlich erschwerte. Andererseits führte die hohe Fremdbetreuungsrate aber auch zu nicht unerheblichen Rekrutierungsschwierigkeiten, da viele der ursprünglich erfassten Kinder bei Personen anderen Namens lebten bzw. selbst anders hießen.

Das höhere Alter der Kinder zum Zeitpunkt der Nachuntersuchung hilft, eine Lücke innerhalb der Forschung zu füllen, da sich die meisten Studien zur Entwicklung von Kindern substanzkonsumierender Mütter auf somatische Variablen zu einem Zeitpunkt kurz nach der Geburt bzw. im Kleinkindalter konzentrieren. Abgesehen von wichtigen Erkenntnissen über 
die Entwicklung der Kinder im Schulalter, zeichnet die vorliegende Studie aber auch ein anschauliches Bild über die Lebensverhältnisse dieser Kinder in Bezug auf ihre Betreuungssituation über den Zeitpunkt kurz nach der Geburt hinaus.

\subsection{Betreuungssituation}

Die Rate der bei den leiblichen Eltern/Müttern lebenden Kinder zum Zeitpunkt der Entlassung betrug innerhalb der Experimentalgruppe ursprünglich $68 \%$ und entspricht gut derjenigen einer anderen deutschen Untersuchung (Bunikowski et al., 1998) sowie einer österreichischen Studie (Berger und Elstner, 2002) mit Raten von 64,7\%, respektive 67,4\%. In unserer Stichprobe lebten zum Zeitpunkt der Untersuchung noch 35\% der Kinder opiatabhängiger Mütter bei zumindest einem leiblichen Elternteil, davon lebten nur noch $45 \%$ bei beiden leiblichen Eltern. Aufgrund des sehr heterogenen Alters unserer Stichprobe zum Zeitpunkt der Nachuntersuchung gestaltet sich ein Vergleich mit der Literatur als schwierig. In den beiden oben genannten Untersuchungen betrug der Anteil der bei den Eltern lebenden Kindern 35,3\% im Alter von einem Jahr (Bunikowski et al., 1998) bzw. 51,2\% in einem Alter von 6 Jahren (Berger et al., 2002), wobei der hohe Prozentsatz in der österreichischen Untersuchung sicherlich auf die positiven Effekte eines im Rahmen der Studie durchgeführten intensiven Betreuungsprogramms zurückzuführen ist. Stellt man sich die Frage nach den Gründen für einen späten Abbruch der Betreuung durch die Eltern, so war zu eruieren, dass einerseits Krankheit und Tod der Mutter eine Rolle spielen, bei einem mindestens ebenso großen Teil der Mütter scheinen aber durch eigene Probleme die inneren Ressourcen zur Betreuung eines Kindes nicht auszureichen und es kommt zur Vernachlässigung und zur freiwilligen Abgabe der Verantwortung, zunächst meistens an die Großmütter; was oft nur eine erste Notlösung ist und worauf noch ein oder mehrere weitere Betreuungswechsel folgen. Die Arbeit von Ziegler (1998) konnte zeigen, dass Mütter von nach der Geburt fremdplatzierten Kindern sich von Müttern nicht fremdplatzierter Kinder deutlich unterscheiden: Der sozioökonomische Status war geringer und das im Krankenhaus dokumentierte Fürsorgeverhalten dieser Mütter war größtenteils mangelhaft. Geht man der Frage nach, ob sich rückwirkend Unterschiede bei den Müttern der verschiedenen Betreuungsgruppen finden lassen und so ein Scheitern in der Erziehungsverantwortung schon absehbar sein könnte, zeigt sich beim Vergleich der Charakteristika der leiblichen Mütter, dass die Mütter der früh fremdplatzierten Kinder erwartungsgemäß die auffälligsten Werte aufwiesen. Die leiblichen Mütter spät fremdplatzierter Kinder (EG3) zeigten eine vergleichbar geringe Kooperation wie die Mütter 
der früh fremdplatzierten Kinder, das Besuchsverhalten war hingegen signifikant besser. Keine signifikanten Unterschiede zeigten sich beim SES und bei der Parität. Nair et al. (1997, 2003) hatten ein jüngeres Alter der Mutter als Risikofaktor für eine Fremdunterbringung identifiziert und auch in der vorliegenden Studie waren die Mütter der EG2 und EG3 qualitativ betrachtet jünger als die Mütter der EG1, der Unterschied war aber nicht signifikant. Als anerkannte Tatsache gilt, dass der Faktor Substitution während der Schwangerschaft die Wahrscheinlichkeit einer Entlassung des Kindes zur leiblichen Mutter deutlich erhöht. In unserer Stichprobe waren demzufolge auch $72 \%$ der Mütter in der EG1 während der Schwangerschaft substituiert vs. nur 21\% der Mütter in der EG2. Die vorliegende Studie zeigt aber auch, dass Substitution nicht vor einem Verlust des Sorgerechts zu einem späteren Zeitpunkt schützt, denn mit 64\% war die Substitutionsrate in der EG3 annähernd so hoch wie in der EG1.

\subsection{Biologische Vulnerabilität und psychosoziale Belastung}

Die ursprüngliche von Ziegler rekrutierte Kontrollgruppe war zur ursprünglichen Experimentalgruppe nach den Kriterien Alter der Mutter bei Geburt, Parität und sozioökonomischer Status parallelisiert. Außerdem handelte es sich um eine medizinische Hochrisikogruppe mit zur Experimentalgruppe vergleichbaren somatischen Komplikationen und hoher Frühgeburtlichkeit. Die hier untersuchte Stichprobe beinhaltet ungefähr die Hälfte dieser Stichprobe von Ziegler und ist sowohl bezüglich der neonatologischen Daten als auch bezüglich der somatischen Risikofaktoren für die ursprüngliche Stichprobe repräsentativ, weshalb auch in unserer Stichprobe die gute Vergleichbarkeit zwischen EG und KG hinsichtlich neonatologischer und körperlicher Variablen gegeben ist. Diese gute Vergleichbarkeit der Kontrollgruppe ist ein entscheidender Vorteil der vorliegenden Studie, denn auch in vielen neueren Studien sind die Kontrollgruppen weder nach psychosozialen noch nach körperlichen Variablen ausreichend gut parallelisiert. Gerade Kinder mit pränataler Drogenexposition haben aber eine erhöhte Wahrscheinlichkeit für Frühgeburtlichkeit und daraus folgend, für ein niedriges Geburtsgewicht, Faktoren, die einen generellen Risikofaktor für Defizite in der weiteren kindlichen Entwicklung darstellen (z.B. Howard et al., 1995; McGauhey, Starfield, Alexander \& Ensminger, 1991; Messinger et al. 2004, Hans \& Jeremy, 2001, Brown, Bakeman, Coles, Sexson \& Demi, 1998; Frank et al., 2002). Fehlt eine diesbezüglich vergleichbare Kontrollgruppe, so sind die potentiellen Auswirkungen unspezifischer körperlicher Risiken nicht von denen drogenspezifischer Risiken zu trennen. 
Auch nach Aufteilung der Stichprobe in vier Gruppen unterschiedlicher Betreuungsverhältnisse zeigten sich bei Geburtsgewicht und Gestationsalter keine Unterschiede. Damit war eine wichtige Voraussetzung erfüllt, um die Auswirkungen der unterschiedlichen postnatalen Erziehungsbedingungen und psychosozialen Risiken auf die Entwicklung von Kindern einer Hochrisikogruppe mit und ohne spezifisches Risiko der pränatalen Opiatexposition zu untersuchen.

Um das Ausmaß des psychosozialen Risikos in den Gruppen zu erfassen, wurde das Interview zur Achse V durchgeführt, wobei sich die Gruppe der Kinder mit spätem Betreuungswechsel gemäß unserer Basisannahme (Hypothese I) eindeutig als die am stärksten belastete Gruppe erwies. Zwischen den anderen Gruppen besteht kein signifikanter Unterschied, deskriptiv betrachtet ist die EG1 nach der EG3 am stärksten belastet, gefolgt von der EG2 und der KG1.

Es entspricht durchaus den Erwartungen, dass die Gruppe der EG1 die zweitstärkste Belastung aufweist. Innerhalb dieser Gruppe gab es zwar keine Bezugspersonenwechsel, dafür wurden aber die suchtmilieuspezifischen psychosozialen Risiken wirksam, die zu einer insgesamt größeren Belastung dieser Kinder führen als für Kinder aus anderen Familien mit niedrigem sozioökonomischen Status (Jacobson \& Jacobson, 2001; Hogan, 1998; Klein, 2003; Englert \& Ziegler, 2001). In dieser Gruppe lebte fast die Hälfte der Mütter von Sozialhilfe, war weiterhin methadonsubstituiert und wies fast durchgängig eine Hepatitis-C Infektion auf. Anhaltender Konsum illegaler Drogen spielte eine kleinere Rolle, allerdings kann davon ausgegangen werden, dass die tatsächliche Rate höher ist als die angegebene. Im Achse V Interview wurden bei einem Großteil der Kinder die Belastungen „Disharmonie in der Familie“ sowie „Psychische Störung/abweichendes Verhalten der Eltern“ identifiziert. Die elterliche Psychopathologie in einer Drogenpopulation gilt als erhöht (Hogan, 1998; Klein, 2003), was auch in der vorliegenden Stichprobe untermauert werden konnte. Deskriptiv betrachtet wiesen die leiblichen Mütter der EG1 im Selbstbeurteilungsfragebogen ASR die stärkste Psychopathologie auf, wobei vor allem die Skalen Sozialer Rückzug, Aufmerksamkeitsprobleme, Regelverletzendes Verhalten, Tabakkonsum und Substanzkonsum allgemein erhöht waren und auch signifikant stärker ausgeprägt als bei den Bezugspersonen der EG2.

Die EG2 ist definiert durch einen frühen Wechsel und ein darauf folgendes konstantes Fremdbetreuungsverhältnis außerhalb eines suchtspezifischen Milieus, womit für diese Kinder innerhalb der Experimentalgruppe sicher die optimalsten Entwicklungsmöglichkeiten vorausgesetzt werden können. Als Einschränkung muss allerdings genannt werden, dass die 
Hälfte der Kinder in dieser Gruppe von den Großmüttern bzw. Großeltern betreut wurde und es zumindest in amerikanischen Studien ein bisher relativ einheitlicher Befund ist, dass bei Kindern drogenabhängiger Mütter verwandtschaftliche Betreuungsverhältnisse den nichtverwandtschaftlichen unterlegen sind (Tyler et al. 1997, Frank et al., 2002. Brown et al., 2004). Inwieweit diese Ergebnisse auf den deutschen Sprachraum übertragbar sind, bleibt fraglich. Die staatlichen und sozialen Unterstützungssysteme sind sicher nicht vergleichbar, andererseits ist aber gut vorstellbar, dass Großeltern aufgrund ihres höheren Lebensalters und ihrer Sorge um das eigene drogensüchtige Kind in ihrer Belastbarkeit und damit in ihrer Erziehungsfähigkeit eingeschränkt sind.

In der EG3 fand der erste von mehreren Betreuungswechseln mit durchschnittlich 24,2 Monaten statt. Über die Umstände der Betreuung bei den Eltern ist in den meisten Fällen wenig bekannt. Aufgrund der Tatsache, dass die Mütter/Eltern die Sorge um ihr Kind abgegeben haben bzw. abgeben mussten, lässt sich allerdings schließen, dass diese Eltern wenig Ressourcen für die Betreuung ihres Kindes hatten und die psychosozialen Umstände vor der Fremdunterbringung wahrscheinlich schlechter waren als bei den Eltern der EG1, die ihre Kinder nach wie vor versorgen.

Die KG1 ist ebenfalls durch ein konstantes Betreuungsverhältnis charakterisiert, die sozioökonomischen Verhältnisse können aber auf Grund der ursprünglichen Parallelisierung als niedrig angenommen werden und waren zum Zeitpunkt der Geburt mit denen der Mütter in der Gruppe EG1 und EG3 vergleichbar. Ein Viertel der Mütter in der KG1 lebt von Arbeitslosengeld oder Sozialhilfe, die HIV-Rate der Mütter in der KG1 war am höchsten.

\subsection{Die Entwicklung der Kinder}

Bezüglich der kognitiven und sozial-emotionalen Entwicklung der Kinder wurde erwartet, dass die Gruppe EG3 aufgrund des Zusammentreffens von biologischer Vulnerabilität und größtem psychosozialen Risiko die stärkste Beeinträchtigung aufweist, während die Kinder der EG2, die bereits kurz nach der Geburt fremdbetreut wurden, trotz biologischer Vulnerabilität eine mit den Kinder der Kontrollgruppe vergleichbare Entwicklung aufweisen. Diese Annahmen wurden gemäß des transaktionalen Modells aufgestellt, wonach die drogenspezifische biologische Vulnerabilität nur einen von vielen Risikofaktoren darstellt und potentiell negative Auswirkungen dieser biologischen Vulnerabilität durch eine fördernde Umwelt und Erziehung kompensiert werden können. Im Rahmen des transaktionalen Modells 
ist es auch möglich, die teilweise sehr widersprüchlichen Forschungsergebnisse $\mathrm{zu}$ integrieren. Es geht weniger um die Frage nach kausalen Wirkzusammenhängen, als vielmehr um die Interaktion verschiedener Einflüsse und deren Beitrag $\mathrm{zu}$ potentiellen Entwicklungsdefiziten.

In dieser Untersuchung bestehen bezüglich der mit dem HAWIK-III gemessenen kognitiven Entwicklung in einer der drei Hauptskalen und in zwei der 11 Subskalen signifikante Unterschiede. Der Handlungs-IQ der EG1 und der EG3 ist annähernd vergleichbar und fällt signifikant geringer aus als der Handlungs-IQ der Kontrollgruppe. Auf Subtestebene finden sich die größten Unterschiede in den beiden zum Handlungs-IQ zählenden Skalen Figurenlegen und Zahlen-Symbol-Test: Alle drei Gruppen mit Opiatexposition erzielen in diesen Skalen geringere Werte als die Kontrollgruppe, der Unterschied von EG1 und EG3 zur Kontrollgruppe ist signifikant, bei der EG2 besteht ein Trend.

Die in Hypothese III angenommene stärkste Beeinträchtigung der EG3 aufgrund des Zusammentreffens von biologischer Vulnerabilität und größtem psychosozialen Risiko lässt sich somit nur teilweise bestätigen, denn die EG1 zeigt zumindest im Bereich der HandlungsIntelligenz ähnlich große Defizite wie die EG3. Auch die EG2 erzielt in den drei relevanten Skalen deutlich niedrigere Werte als die Kontrollgruppe, wodurch die in Hypothese IV vorausgesagte vergleichbar gute Entwicklung von EG2 und KG1 in Bezug auf die kognitive Entwicklung nicht durchgängig bestätigt werden kann. Geht man davon aus, dass in der Gruppe EG2 relativ gute und zur KG1 mindestens vergleichbare postnatale Erziehungsbedingungen herrschen, diese Gruppe aber speziell bei Aufgaben der Handlungsintelligenz - also Anforderungen die durch Umwelteinflüsse deutlich weniger beeinflusst werden können als die Verbalintelligenz - geringere Werte aufweisen als die Gruppe KG1, so kann dies als Hinweis auf die Auswirkung einer biologischen Vulnerabilität interpretiert werden, die durch verbesserte Umweltbedingungen nicht vollständig kompensiert werden kann. Ergebnisse, die ähnlich interpretiert werden können, stammen in Bezug auf Opiatkonsum von Moe \& Slinning (2001); für Kokainkonsum von Koren et al. (1998) und Nulman et al (1994). Genauso gibt es aber auch eine Reihe von Untersuchungen, die in Bezug auf die kognitive Entwicklung keinerlei Unterschiede zwischen adoptieren Kindern und bei ihren Eltern lebenden Kindern ausmachen konnten (z.B. Ornoy et al., 1996). Möglicherweise spielt hier eine Rolle, dass in der Gruppe EG2 anders als in der Studie von Ornoy et al. die Übermittlung in die Fremdbetreuung oft nicht sofort nach der Geburt erfolgte, wodurch die 
Umweltbedingungen insgesamt nicht ganz so optimal sind wie bei einer Übermittlung direkt nach der Geburt. Unsere Ergebnisse unterstützen nicht weiter oben berichtete Ergebnisse, dass verwandtschaftliche Pflegeverhältnisse gegenüber nichtverwandtschaftlichen schlechtere Entwicklungsbedingungen für die Kinder bedeuten. Bei einem nachträglich durchgeführten (nicht im Ergebnisteil dargestellten) Gruppenvergleich innerhalb der Gruppe EG2 zwischen den gleich großen Gruppen der Großeltern einerseits und den Pflege-/Adoptiveltern andererseits unterschieden sich die Kinder in der kognitiven Entwicklung nicht signifikant voneinander; qualitativ betrachtet erzielten die von den Großeltern betreuten Kinder sogar bessere Ergebnisse.

Gut untermauert werden können hingegen Ergebnisse (z.B. Chasnoff et al., 1998; Griffith et al., 1994; Tronick \& Beeghly, 1999, Messinger et al., 2004), wonach die durchschnittliche Leistung an den unteren Rand des Normbereichs verschoben war. In der vorliegenden Studie sind die durchschnittlichen IQ-Werte für die Gruppen EG1 und EG3 im Handlungs-IQ und Gesamt-IQ um ca. eine Standardabweichung nach unten verschoben. Diese Verschiebung nach unten zeigt sich auch in der Verteilung der IQ-Werte, näherungsweise eine Normalverteilung findet sich nur in der KG1.

Innerhalb der Gruppe der leiblichen Mütter zeigte sich ein positiver Zusammenhang zwischen höherer Schulbildung der Mutter und besserer Intelligenztestleistungen der Kinder. Da sich die Schulbildung der Mütter der KG1 und EG1 aber nicht voneinander unterschieden und eine Kovarianzanalyse mit der Schulbildung als Kovariate nichts an der Signifikanz der Ergebnisse änderte, kann ausgeschlossen werden, dass das schlechtere Abschneiden der Kinder mit Opiatexposition in Skalen der Handlungskompetenz in potentiell niedrigeren kognitiven Fähigkeiten ihrer leiblichen Mütter und damit durch hauptsächlich genetische Effekte bedingt sein könnte.

Ebenfalls kann ausgeschlossen werden, dass die Unterschiede durch Ausreißereffekte bedingt sind. Die Ergebnisse blieben auch bei einer Wiederholung der Kovarianzanalyse ohne Einschluss des IQ-Wertes über 130 in der KG1 signifikant.

Die Annahme (Hypothese II) eines Zusammenhangs zwischen der Schwere des neonatalen Abstinenzsyndroms und der kognitiven Entwicklung (Berger \& Elstner, 2002) wurde bestätigt: Je ausgeprägter die Entzugssymptomatik ist, desto niedriger ist die kognitive Leistung. Ebenfalls bestätigt wurde, wie in der Literatur oft berichtet, dass die Substitution in der Schwangerschaft ein stärkeres Entzugssyndrom nach sich zieht. Der Unterschied in unserer Stichprobe war signifikant. Die Schwere des NAS hatte aber keinen moderierenden 
Einfluss auf die Unterschiede zwischen den Gruppen EG1, EG2 und EG3, da das NAS in allen drei Gruppen durchschnittlich gleich stark ausgeprägt war. Es zeigten sich auch keine signifikanten Unterschiede zwischen der Intelligenztestleistung der Kinder substituierter und nicht-substituierter Mütter. Qualitativ betrachtet erzielten die nicht-substituierten Kinder aber einen etwas höheren Gesamt-IQ als die substituierten Kinder. Nicht unterstützt wird diese Tendenz von den Ergebnissen von Bunikowski et al. (1998), in deren Studie kein Zusammenhang zwischen der Dauer der Phenobarbitalgabe, die mit der Schwere des NAS korreliert war, und dem durchschnittlichen Entwicklungsquotienten in den Griffiths Skalen gefunden wurde und die substituierte Gruppe in der Subskala Leistungen besser abschnitt als die nicht-substituierte Gruppe. Einschränkend muss gesagt werden, dass die hier verwendete Stichprobengröße klein ist, die Studie nicht auf die Erforschung dieser Zusammenhänge ausgelegt war und die gefundenen Effekte natürlich auch stark durch in diesem Zusammenhang nicht näher kontrollierte, psychosoziale Einflüsse überlagert sind.

Erstaunlicherweise fanden sich weder in der Gruppe der Kinder mit pränataler Opiatexposition noch in der Kontrollgruppe statistisch relevante Zusammenhänge zwischen Geburtsgewicht und kognitiver Entwicklung.

Bei der sozial-emotionalen Entwicklung (CBCL) weist die EG3 im Großteil der CBCLPsychopathologie-Skalen eine stärkere Beeinträchtigung auf als EG2 und KG1. Anders als in der Hypothese III formuliert und vergleichbar mit den Ergebnissen zur kognitiven Entwicklung, ist die EG3 aber nicht schwerer belastet als die EG1 und der Unterschied von EG1 zu EG2 und KG1 ist teilweise ebenfalls signifikant. Besonders groß sind die Unterschiede von EG1 und EG3 zu KG1 (ca. 1 Standardabweichung) in den Skalen Gesamt, Externalisierend, Soziale Probleme und Aufmerksamkeitsprobleme. Aufgrund der angenommenen ungünstigeren postnatalen Erziehungsbedingungen in den Gruppen EG1 und EG3 ist dies vor allem als Effekt einer wenig förderlichen Umwelt zu interpretieren. Besonders negativ sind in diesem Zusammenhang auch wiederholte Bezugspersonenwechsel und die Anzahl der Betreuungswechsel ist zur CBCL-Skala Externalisierend positiv korreliert. Die Hypothese IV, wonach pränatal opiatexponierte Kinder, die bereits kurz nach der Geburt fremdbetreut werden, im Vergleich zu Kindern der Kontrollgruppe eine vergleichbare Entwicklung nehmen, konnte mit zwei Ausnahmen durchgängig bestätigt werden: Bei der Aufmerksamkeitsproblematik besteht ein Trend hinsichtlich einer stärkeren Auffälligkeit der EG2 gegenüber der KG1 und beim Aggressiven Verhalten ist die EG2 signifikant auffälliger 
als die KG1. Diese Ergebnisse lassen sich durch bisherige Ergebnisse aus der Literatur gut erklären, wonach Kinder aus Drogenfamilien erhöhte Psychopathologiewerte haben, insbesondere auch in den Bereichen Externalisierend und Aufmerksamkeitsproblematik (Wilens et al. 1995; Griffith et al., 1994; Phelps et al., 1997; Richardson, 1998), bzw. Kinder auch bei Aufmerksamkeitsanforderungen schlechter abschneiden (Mayes et. al., 1998). Es ist auch bekannt, dass innerhalb der Suchtpopulation die ADHD-Raten stark erhöht sind (z.B. Rounsaville et al., 1991, Horner et al., 1997) und Drogen oft im Sinne einer Selbstmedikation verwendet werden - eine erbliche Komponente spielt hier mit großer Wahrscheinlichkeit eine Rolle.

Die größere internalisierende Symptomatik bei den Jungen ist ein überraschendes Ergebnis und eine größere Anfälligkeit von Jungen wurde bisher eher in Bezug auf kognitive Fähigkeiten berichtet (Moe \& Slinning, 2001).

Eine erhöhte Psychopathologie der Eltern gilt als Risikofaktor für die Entwicklung der Kinder (z.B. Howard, 1995; Jacobson \& Jacobson, 2001) und für die untersuchte Stichprobe konnten diese Ergebnisse gut untermauert werden: In der Gruppe der leiblichen Eltern (EG1 und KG1), in der ein kontinuierliches Betreuungsverhältnis bestand, zeigte sich ein positiver Zusammenhang zwischen der Psychopathologie der Mutter im ASR und den CBCL-Skalen Gesamt, Internalisierend und Externalisierend. In einer Kovarianzanalyse innerhalb der Gruppe der leiblichen Eltern trug die mütterliche Psychopathologie in zumindest zwei Skalen wesentlich zu den Gruppenunterschieden bei.

Bei den bisherigen Konsultationen aufgrund psychischer Probleme nehmen die Adoptiv- bzw. Pflegeeltern in den Gruppen EG2 und EG3 die Spitzenposition ein, was nicht mit der in der CBCL beschriebenen geringeren Auffälligkeit der EG2 übereinstimmt. Eine Erklärung dafür ist das allgemein bekannte Phänomen (Warren, 1992), dass Adoptiv- und Pflegeeltern eine höhere Wachsamkeit und Bereitschaft zeigen, bei sich abzeichnenden emotionalen Problemen professionelle Hilfe in Anspruch zu nehmen; Mütter mit einer Drogenproblematik zeigen diesbezüglich hingegen scheinbar eine große Zurückhaltung. Von der Scheu offizielle Stellen zu konsultieren ist die Bereitschaft zur Beschreibung der Verhaltensauffälligkeiten aber unabhängig (Simmel et al., 2001), und auch in unserer Studie berichteten die Eltern der EG1, die nur zu einem sehr geringen Teil professionelle Hilfe in Anspruch genommen hatten, über deutlich mehr Verhaltensauffälligkeiten als die EG2. 
Beim GAF, als Maß der psychosozialen Anpassung, gibt es gemäß unserer hypothetischen Annahmen keine Unterschiede zwischen EG2 und KG1 und es besteht ein Trend hinsichtlich eines signifikant schlechteren psychosozialen Funktionsniveaus der EG3 gegenüber allen anderen Gruppen.

Der Summenwert der Achse V war bestimmt zur Absicherung unserer in Hypothese I getroffenen Annahme der hohen Belastung der EG3 und wurde in den Varianzanalysen nicht als Kovariate berücksichtigt, da die Existenz eines hohen psychosozialen Risikos bereits in der Definition der Gruppe EG3 enthalten war - hätten bei Miteinbeziehung als Kovariate signifikante Ergebnisse ihre Signifikanz verloren, hätte das zu einem Zirkelschluss geführt. Trotzdem sollte auf den Aussagewert einer kontinuierlichen Variable nicht verzichtet werden, weshalb zur besseren Veranschaulichung noch eine abschließende Regressionsanalyse berechnet wurde. Statt des Faktors Gruppe dienten (neben Alter und Geschlecht) der Summenwert der Achse V und die Variable ,pränatale Drogenexposition ja/nein“ als Prädiktoren. Hervorzuheben ist, dass der Faktor „pränatale Drogenexposition ja/nein“ natürlich nicht mit dem Vorhanden- oder Nichtvorhandensein eines biologischen Risikos gleichgesetzt werden darf, da in diesem Faktor natürlich auch mögliche ungünstige psychosoziale Begleitumstände enthalten sind. Variablen, die sich in den vorangegangenen Analysen als relevant erwiesen haben, wie die Schwere des Entzugssyndroms und die mütterliche Psychopathologie, konnten, da sie immer nur für Subgruppen vorhanden waren, in der Gesamtanalyse leider nicht einbezogen werden. Das Ergebnis der Regression fasst die Ergebnisse der Gruppenvergleiche quasi in einem Bild zusammen: Während bei den kognitiven Fähigkeiten die Zugehörigkeit zur Gruppe mit Drogenexposition der relevanteste Prädiktor ist, leisten bei der CBCL und beim GAF die Ausprägung der psychosozialen Risiken (Achse V) den jeweils wichtigsten Beitrag. Die externalisierende Symptomatik ist zusätzlich auch durch den Faktor Drogenexposition, die internalisierende Symptomatik auch durch das Geschlecht des Kindes bestimmt.

Zur Schwere der psychosozialen Belastung in den Gruppen ist abschließend zu sagen, dass die Gruppe EG3 in der Achse V zwar die stärkste Belastung aufweist, in keinem einzigen Bereich resultiert dies aber in einer entsprechend stärkeren Beeinträchtigung in der Entwicklung als in der EG1. Die Gruppe EG1 hat zwar keine Traumatisierungen aufgrund mehrmaliger Bezugspersonenwechsel erlebt, der maligne Einfluss durch das Drogenmilieu, 
wie z.B. die erhöhte Psychopathologie der Mutter oder deren anhaltender Drogenkonsum sind aber in ihren Auswirkungen den Risiken der EG3 vergleichbar.

Die vorliegende Arbeit ist eine von ganz wenigen Studien zu den Auswirkungen des pränatalen Opiatkonsums bei Kindern jenseits des Kleinkindalters und unter Verwendung einer gut parallelisierten Kontrollgruppe. Trotzdem gibt es auch bei dieser Untersuchung eine Reihe von Limitierungen:

Die Größe der Stichprobe ist relativ klein.

Daten der leiblichen Eltern sind nur für die Gruppen EG1 bzw. KG1 (z.B. mütterliche Psychopathologie) oder gar nicht (z.B. mütterliche Intelligenz) vorhanden, so dass auf die genauere Untersuchung eines möglichen genetischen Effekts verzichtet, bzw. auf Kompromisslösungen zurückgegriffen werden musste, wie dies z.B. durch die Kontrolle eines potentiell genetischen Einflusses auf die kindliche kognitive Entwicklung durch die mütterliche Schulbildung von EG1 und KG1 geschehen ist.

Weder das Ausmaß des Opiatkonsums noch das Ausmaß des sehr wahrscheinlichen Beikonsums anderer Substanzen wie Alkohol, Benzodiazepine, Barbiturate und vor allem Nikotin konnten aufgrund der retrospektiven Stichprobenrekrutierung erfasst werden, so dass über die Wirkung unterschiedlicher Dosierungen, das Ausmaß des Beikonsums und möglicher synergistischer Effekte auf die Entwicklung keine Aussagen gemacht werden können.

Nur die Untersucherin, die den HAWIK-III durchführte, war dem jeweiligen Gruppenstatus gegenüber blind, bei allen anderen Interviews verfügte die Untersucherin aber über diese Information, so dass, auch wenn eine unter diesen Umständen größtmögliche Objektivität angestrebt wurde, Versuchsleitereffekte nicht gänzlich auszuschließen sind.

Das Interview zur Achse $\mathrm{V}$ hat sich in dieser Studie als sehr praktikables Instrument erwiesen, das trotz heikler Fragen bei den befragten Personen auf eine durchgängig große Akzeptanz traf, und durch dessen Anwendung ein umfassendes Bild über die psychosoziale Gefährdung erstellt werden konnte. Trotzdem waren aufgrund der Gegebenheiten dieser Studie damit auch Schwierigkeiten verknüpft:

- Die Befragung erfolgte retrospektiv für den gesamten Lebenszeitraum, was bedeutet, dass die Angaben in der Gruppe EG3 für frühere Lebensabschnitte zum großen Teil auf Wissen aus zweiter Hand beruhen, da die Bezugsperson zum Untersuchungszeitpunkt in seltenen Fällen die gleiche war wie in früheren 
Lebensabschnitten. Über- und Unterschätzungen der Belastung auf der Achse V können die Folge sein.

- Trotz guter Akzeptanz des Interviews besteht aufgrund der Erkenntnisse einer Vielzahl von Studien, die eine hohe Rate von Kindesmisshandlung und -vernachlässigung bei drogenkonsumierenden Eltern berichten, die begründete Annahme, dass die tatsächliche Rate von Kindesmisshandlung besonders in der EG1 größer ist, als die berichtete.

Der Fokus dieser Arbeit lag auf den Auswirkungen unterschiedlicher Betreuungsverhältnisse, womit die Frage nach möglichen Empfehlungen für das Vorgehen der Jugendhilfe, insbesondere in Bezug auf die Initiierung von Fremdunterbringungen, auf der Hand liegt. Aufgrund der deutlichen Risiken, die mit einem Verbleib der Kinder bei ihren leiblichen Müttern verbunden sind, kann sicher geraten werden, bei Vorhandensein guter Gründe bei der Frage nach einer Fremdunterbringung nicht unnötig zögerlich zu sein. Die Empfehlung für quasi prophylaktische Fremdunterbringungen bedeutet dies aber natürlich nicht, denn die Entwicklungsverläufe sind zu individuell. Ob es zu einem günstigen oder ungünstigen Verlauf kommt, lässt sich nicht absehen und natürlich ist der Verbleib der Kinder bei ihren Eltern unter ,vertretbaren“ Umständen zu fördern. Um diese Umstände zu verbessern, zeichnet sich aber die Notwendigkeit ab, sowohl den betroffenen Eltern, vor allem aber den betroffenen Kindern, frühzeitig und lange bevor eine mögliche Fremdunterbringung im Raum steht, Hilfe zukommen zu lassen, da, als Fazit dieser Untersuchung, die psychosozialen und suchtmilieuspezifischen Risiken in ihren Auswirkungen vor allem die sozial-emotionale Entwicklung der Kinder deutlich beeinträchtigen können. 


\section{Zusammenfassung}

Die vorliegende Studie hatte zum Ziel die kognitive und sozial-emotionale Entwicklung von Kindern substanzabhängiger Mütter zu untersuchen. Dies geschah unter Berücksichtigung verschiedener medizinischer und psychosozialer Einflussfaktoren und unter dem speziellen Aspekt unterschiedlicher Betreuungsbedingungen. Die hier untersuchte Stichprobe wurde aus einem retrospektiv erhobenen Datensatz von Ziegler (1998) rekrutiert. Dieser Ausgangsdatensatz umfasste alle Fälle von Neugeborenen, bei denen in den Jahren 1988 1995 in der Abteilung für Neonatologie des Universitätsklinikums der Johann Wolfgang Goethe-Universität, Frankfurt am Main, eine pränatale Opiatexposition, d.h. Heroin oder Methadon, festgestellt worden war. Zusätzlich war eine nach Alter der Mutter, Parität und sozioökonomischen Status parallelisierte Kontrollgruppe rekrutiert worden, die auch hinsichtlich der somatischen Risiken (Frühgeburtlichkeit, postnatale Komplikationen) zur Gruppe der pränatal opiatexponierten Kinder vergleichbar war.

Von diesen insgesamt 151 Kindern (101 Opiat-, bzw. Experimentalgruppe, 50 Kontrollgruppe) konnte mit insgesamt 57 Kindern der Experimentalgruppe und 21 Kindern der Kontrollgruppe Kontakt aufgenommen werden, von 50 bzw. 18 Kindern sind vollständige Datensätze vorhanden. Unter dem Aspekt der unterschiedlichen Betreuungsbedingungen wurden diese Kinder in insgesamt vier Gruppen aufgeteilt: Die Gruppe EG1 (Eltern) umfasste alle Kinder mit pränataler Opiatexposition, die kontinuierlich bei mindestens einem leiblichen Elternteil lebten oder aktuell leben, die Gruppe EG2 (Fremdbetreuung zu einem frühen Zeitpunkt) umfasste alle Kinder mit pränataler Opiatexposition, die schon früh zu Pflege/Adoptiveltern oder den Großeltern überstellt wurden, die Gruppe EG3 (Fremdbetreuung zu einem späten Zeitpunkt) beinhaltete alle Kinder mit pränataler Opiatexposition, die erst nach dem ersten Lebensjahr in eine Fremdbetreuung überstellt worden waren, bzw. mehrere Bezugspersonenwechsel erlebt hatten und die Gruppe KG1 (Kontrollgruppe Eltern) umfasste alle Kinder der Kontrollgruppe ohne pränatale Opiatexposition, die bei mindestens einem leiblichen Elternteil aufwachsen.

Diese vier Gruppen wurden hinsichtlich der Kriterien Intelligenz (Hamburg-WechslerIntelligenz-Test HAWIK-III) und der sozial-emotionalen Entwicklung (Child Behavior Checklist CBCL) und psychosozialem Funktionsniveau (Global Assessment Functioning 
Scale GAF) miteinander verglichen. Relevante Einflussfaktoren wurden dabei kontrolliert. Als Ergebnisse dieser Gruppenvergleiche kann festgehalten werden:

Im HAWIK-III erzielen die Gruppen EG1 und EG3 im Handlungs-IQ und in zwei Subskalen des Handlungsteils, nämlich Figurenlegen und Zahlen-Symbol-Test, signifikant schlechtere Ergebnisse als die KG1. Bei der EG2 besteht ein Trend hinsichtlich eines signifikant schlechteren Abschneidens gegenüber der KG1 in den beiden Subskalen. Vor allem die Ergebnisse des Handlungs-IQ sind in den Gruppen EG1, EG2 und EG3 um durchschnittlich eine Standardabweichung niedriger als es der Norm entspricht.

In der CBCL sind die Gruppen EG1 und EG3 am stärksten belastet, in vielen der Skalen sind die Unterschiede zur Gruppe KG1 und teilweise auch zur EG2 signifikant. In fast allen Skalen ist die psychopathologische Auffälligkeit von EG2 und KG1 vergleichbar, eine Ausnahme bilden dabei aber die Skalen Aufmerksamkeitsproblematik und Aggressives Verhalten, in denen die EG2 auch auffälligere Werte als die KG1 zeigt.

Im GAF besteht ein Trend hinsichtlich eines signifikant schlechteren psychosozialen Funktionsniveaus der EG3 gegenüber allen anderen Gruppen.

Insgesamt können die Ergebnisse im Sinne des transaktionalen Modells interpretiert werden, wonach die pränatale Drogenexposition als ein Risikofaktor innerhalb der Entwicklung gesehen wird, der durch andere Risiko- und Schutzfaktoren beeinflussbar ist. Die Unterschiede in den Gruppen sind durch nachfolgende Umwelteinflüsse maßgeblich determiniert und in der EG1 und EG3, in denen das höchste psychosoziale Risiko besteht, finden sich auch die größten Entwicklungsbeeinträchtigungen. Durch fördernde Umweltbedingungen wie in der EG2 ist die Entwicklung hingegen mit der KG1 vergleichbar. Speziell für die Entwicklung von Aufmerksamkeitsproblemen und aggressivem Verhalten, bzw. bei Anforderungen manuell-visueller Handlungskompetenz, finden sich Hinweise auf eine erhöhte Vulnerabilität bei Kindern mit pränataler Opiatexposition, die auch durch fördernde Umweltbedingungen nicht vollständig kompensiert werden kann. $\mathrm{Ob}$ diese spezifische Vulnerabilität in Auswirkungen der Opiate an sich oder in genetischen Einflüssen begründet ist, lässt sich allerdings nicht beantworten.

Diese Studie zeichnet ein umfassendes Bild über die Situation von Kindern opiatabhängiger Mütter, einerseits hinsichtlich der Betreuungssituation, andererseits hinsichtlich der kognitiven und sozial-emotionalen Entwicklung. Viele Fragen bleiben aber offen und eine 
follow-up Untersuchung dieses Kollektivs könnte Erkenntnisse über die Interaktion der relevanten Einflussfaktoren auf die kindliche Entwicklung bringen. Ein Fokus sollte hierbei vor allem auf der Aufmerksamkeit, aggressivem und impulsivem Verhalten sowie auf einer potentiellen Suchtentwicklung der Kinder und Jugendlichen selbst liegen. 


\section{Summary}

The objective of this study is to assess the cognitive and social-emotional development of children born to opiate-addicted mothers. Different medical and psychosocial confounding variables are considered, especially the aspect of the postnatal care giving environment. The children in the present study are part of a retrospective sample survey of Ziegler (1998). This initial sample contained all cases of newborn children in the University of Frankfurt, Germany, with prenatal opiate-exposure, which could be Heroine or Methadone. Also a control group was recruited, whose participants were comparable on maternal age, parity and socioeconomical status.

Of these initially 150 children (101 children with opiate-exposure, 50 children in the control group) 57 of the opiate-exposed (experimental group) and 21 of the control group could be contacted. A full set of data exists for 50 respectively 18 children of these two groups. Focusing on the importance of the postnatal care giving environment the children were divided into four groups: Group EG1 (parents) contained all children with prenatal opiateexposure who continuously lived or still live with at least one biological parent. Group EG2 (early non-parental care) comprises all children with prenatal opiate-exposure who became cared for by foster parents, adoptive parents or grandparents at a very early point of time in their life. Group EG3 (late non-parental care) comprises all children with prenatal opiateexposure who were transferred into non-parental care after their first birthday or who experienced several changes of their primary caregiver. The fourth group (KG1, control group) contained all children without prenatal opiate exposure who continuously grew up with at least one biological parent.

The children of those four groups were assessed on cognitive functioning (HAWIK-III), social-emotional development (Child Behavior Checklist, CBCL) and level of psychosocial functioning (Global Assessment Scale, GAF). Relevant confounding variables were controlled.

On the HAWIK-III Performance-IQ and two subscales of the Performance Scale, Coding and Object Assembly, children of group EG1 and EG3 scored significantly lower than group KG1. EG2 showed a trend toward lower results in the two subscales than group KG1. In the groups EG1, EG2 and EG3 scores of the performance-IQ are on average one standard deviation below the normative standards. On the CBCL groups EG1 and EG3 showed higher 
scores on the psychopathology scales than the groups KG1 and EG2, with reaching significance in many of the subscales.

In almost all subscales KG1 and EG2 did not differ, except Attention Problems and Aggressive Behaviours, in which children of EG2 were more likely to score in the clinically significant range.

On the GAF group EG3 showed a trend toward a significant lower level of psychosocial functioning than the three other groups.

The presented results are in line with a transactional risk model, in which prenatal drugexposure is seen as one risk factor within child development, which is influenced by other risk as well as protective mechanisms. Group differences are determined through subsequent environmental influences. Therefore children of groups EG1 and EG3, who experienced the highest psychosocial stress, also showed the most developmental deficits. Through optimization of postnatal conditions as in group EG2 development was comparable to group KG1. Especially for the risk of developing attention problems, aggressive behaviour and difficulties in perceptual-motor performance, there seems to be a higher vulnerability for children with prenatal drug-exposure, that can not be fully compensated for by an optimized care-giving environment.

The results of the presented study add important detail about the situation of children of opiate abusing mothers, about care-giving conditions and cognitive and social-emotional development. Still many questions remain open and a follow-up study could help to better understand the interaction of potential risk and protective/promotive factors on child development. Future research should especially emphasize on attention problems, aggressive behaviour and impulsivity as well as on a potential development of substance abuse of the children themselves. 


\section{Literaturverzeichnis}

Alessandri, S.M., Bendersky, M., \& Lewis, M. (1998). Cognitive Functioning in 8- to 18month old drug-exposed infants. Developmental Psychology, 34, 565-573

Alessandri, S.M., Sullvian, M.W., Imaizumi, S. \& Lewis, M. (1993). Learning and emotional responsivity in cocaine-exposed infants. Developmental Psychology. 29, 989-997.

Arbeitsgruppe deutsche Child Behavior Checklist (1997). Adult Self Report (ASR). Klinik für Kinder- und Jugendpsychiatrie der Universität zu Köln: Arbeitsgemeinschaft Kinder-, Jugendlichen- und Familiendiagnostik (KJFD)

Arendt, R.E., Short, E.J., Singer, L.T., Minnes, S., Hewitt, J., Flynn, S., Carlson, L., Min, M.O., Klein, N. \& Flannery, D. (2004). Children prenatally exposed to cocaine: developmental outcomes and environmental risks at seven years of age. Developmental and Behavioural Pediatrics, 25, 83-90.

Arnold, T., Feldmeier-Thon, J., Fritsch, R. \& Simmedinger, R. (1995). Wem hilft Methadon? Daten, Fakten, Analysen. Frankfurt: Institut für Sozialarbeit und Sozialpädagogik (ISSReferat 1/1995)

Bada, H.S., Verter, J., Shankaran, C.R., Lester, B., Wright, L.L., Smeriglio, V.L., Finnegan, L.P. \& Maza, P.L. (1996). Maternal lifestyle study (MSL): Intrauterine growth of infants exposed to cocaine/opiates $(\mathrm{C} / \mathrm{O})$ in utero. Pediatric Research, 35, 256A

Bandstra, E.S., Morrow, C.E., Vogel, A.L., Fifer, R.C., Ofir, A.Y., Dausa, A.T., Xue, L. \& Anthony, J.C. (2002). Longitudinal influence of prenatal cocaine exposure on child language functioning. Neurotoxicology and Teratolgoy, 24, $297-308$.

Bauman, P.S. \& Levine, S.A. (1986). The development of children of drug addicts. The International Journal of the Addictions, 18, 291-302

Bästlein, M. \& Poustka, F. (1993). Anwendbarkeit und Reliabilität der psychosozialen Achse der WHO. In: F. Poustka \& U. Lehmkuhl (Hrsg.): Gefährdung der kindlichen Entwicklung. Quintessenz, München.

Beeghly, M., Frank, D.A., Rose-Jacobs, R., Cabral, H. \& Tronick, E. (2003). Level of prenatal exposure and infant-caregiver attachment behaviour. Neurotoxicology and Teratology, 25, 23-38. 
Belsky, J. \& Pasco Fearon, R.M. (2002). Infant-mother attachment security, contextual risk, and early development: A moderational analysis, Developmental and Psychopathology, 14, 293-310.

Bendersky, M, \& Lewis, M. (1998). Arousal modulation in cocaine-exposed infants. Developmental Psychology, 34, 555-564.

Berger, E. \& Elstner, T: Entwicklung von Kindern substanzabhängiger Mütter. Endbericht im Mai 2002. Kurzfassung. In http://www.univie.ac.at/kjnp-rehab-integra/projekt/kinder _substanzabhaengiger_muetter_kurz.htm (5.12.2004)

Bortz, J. (1993). Statistik. Für Sozialwissenschaftler. Berlin: Springer-Verlag.

Bernstein, V.J., Jeremy, R.J. \& Marcus, J. (1986). Mother-infant interaction in multiproblem families: Finding those at risk. Journal of the American Academy of Child Psychiatry, 25, $631-640$

Berrick, J.D., Barth, R.P. \& Needell, B. (1994). A comparison of kinship foster homes and foster family homes: Implications for kinship foster care as family preservation. Children and Youth Services Review, 16, 33-63.

Besinger, B., Garland, A.F., Litrownik, A.J. \& Landsverk, J.A. (1999). Caregiver substance abuse among maltreated children placed in out-of-home care. Child Welfare, 78, 221-239.

Bunikowski, R., Grimmer, I., Heiser, A., Metze, B., Schäfer, A. \& Obladen, M. (1998). Neurodevelopmental outcome after prenatal exposure to opiates. European Journal of Pediatrics, 157, 724-730.

Brown, J.V., Bakeman, R., Coles, C.D., Demi, A.S. \& Sexson, W.R. (1994). Neonatal outcome in infants exposed to cocaine: Are preterms more severely affected than fullterms? Pediatric Research, 35, 27A

Brown, J.V. Bakeman, R., Coles, C.D., Sexson, W.R \& Demi, A.S. (1998). Maternal drug use during pregnancy: are preterm and full-term infants affected differently? Developmental Psychology, 34, 540-554.

Brown, J.V., Bakeman, R., Coles, C.D., Platzman, K.A. \& Lynch, E.M. (2004). Prenatal cocaine exposure: a comparison of 2-year-old children in parental and nonparental care. Child Development, 75, 1282-1295. 
Chaffin, M., Kelleher, K. \& Hollenberg, J. (1996). Onset of physical abuse and neglect: psychiatric, substance abuse, and social risk factors from prospective community data. Child Abuse \& Neglect, 20, 191-203.

Charvoz, L., Bodenmann, G., \& Hermann, E. (2002). Streß- und Risikoforschung. Die Bedeutung der Familie für den Konsum psychotroper Substanzen bei Jugendlichen. Kindheit und Entwicklung, 11, 14-20.

Chasnoff, I.J., Anson, A., Hatcher, R., Stenson, H., Iaukea, K. \& Randolph, L.A. (1998). Prenatal exposure to cocaine and other drugs. Outcome at four to six years. In: Harvey, J.A. \& Kosofsky, B.E. (Hrsg): Cocaine. Effects on the developing brain (S 314-328) (Annals of the NYAS Vol 846). New York, NY: The New York Academy of Sciences.

Colten, M.E. (1980). A comparison of heroin-addicted and non-addicted mothers: Their attitudes, beliefs and parenting experiences. NIDA, Services Research Report: Heroin addicted parents and their children. National Institute on drug abuse, U.S. Departement of Health and Human Services (Pub. No. 81-1028). Washington, D.C.: US Government Printing Office.

Davis, S.K. (1990). Chemical dependency in women: A description of its effects and outcome on adequate parenting. Journal of Substance Abuse Treatment, 7, 225-232.

De Cubas, M.M. \& Field, T. (1993). Children of methadone-dependent women: developmental outcomes. American Journal of Orthopsychiatry, 63, 266-276

Delaney-Black, V., Covington, C., Templini, T., Ager, J., Nordstrom-Klee, B., Martier, S., Leddick, L., Czerwinski, R.H. \& Sokol, R.J. (2000). Teacher-assessed behavior of children prenatally exposed to cocaine. Pediatrics, 106, 782- 791.

Delmo, C., Weiffenbach, O., Gabriel, M., Bölte, S., Marchio, E. \& Poustka, F. (1998). Kiddie-SADS-Present and Lifetime Version (K-SADS-PL). 2. Auflage der deutschen Forschungsversion. Frankfurt: Eigendruck.

Deutsches Bundesministeriums für Gesundheit (2005). Drogen- und Suchtbericht 2005

Döpfner, M., Melchers, P., Fegert, J., Lehmkuhl, G., Lehmkuhl, U., Schmeck, K., Steinhausen, H.-CH. \& Poustka, F. (1994). Deutschsprachige Konsensus-Versionen der Child Behavior Checklist (CBCL/4-18), der Teacher Report Form (TRF) und der Youth Self Report Form (YSR). Kindheit und Entwicklung. 3, 54-59. 
Dubowitz, H., Feigelman, S., Harrington, D., Starr, R., Zuravin, S. \& Sawyer, R. (1994). Children in kinship care: How do they fare? Children and Youth Services Review, 16, 85-106. Englert, E. \& Poustka, F. (1995). Das Frankfurter Kinder- und Jugendpsychiatrische Dokumentationssystem - Entwicklung und methodische Grundlagen unter dem Aspekt der klinischen Qualitätssicherung. Praxis der Kinderpsychologie und Kinderpsychiatrie, 44, 158167.

Englert E. \& Ziegler, M. (2001). Kinder opiatabhängiger Mütter - Ein Überblick. Suchttherapie, 2, 143-151.

Egami, Y., Ford, D.E., Greenfield, S.F. \& Crum, R.M. (1996). Psychiatric profile of sociodemographic characteristics of adults who report physically abusing or neglecting children. American Journal of Psychiatry, 153, 921-928.

Fanaroff, A.A. \& Martin, R.J. (2002). Neonatal-perinatal medicine. Diseases of the Fetus and Infant. Philadelphia, S 661.

Fanshel, D. (1975). Parental failure and consequences for children. The drug-abusing mother whose children are in foster care. American Journal of Diseases in Children, 65, 604-612.

Finnegan, L.P. (1985). Neonatal abstinence. In Nelson, N.M. (Hrsg). Current therapy in neonatal-perinatal medicine (2ed), 262-270.

Finnegan, L.P. (2000). Women, pregnancy and methadone. Heroin Addiction \& Related Clinical Problems, 2, 1-8.

Finnegan, L.P. \& Ehrlich, S.M. (1990). Maternal drug abuse during pregnancy: Evaluation and pharmacotherapy for neonatal abstinence. Modern methods in pharmacology, 6, 225 263.

Finnegan, L.P \& Kandall, S.R. (1997). Maternal and neonatal effects of alcohol and drugs. In Lowinson, J.H., Ruiz, P., Millman, R.B. \& Langrod, J.G. (Hrsg.). Substance Abuse - A Comprehensive Textbook. Section VII. Management of Medical Conditions Associated with Substance Abuse, (S 513-534). Baltimore, Williams \& Wilkins.

Fitzgerald, E., Kaltenbach, K. \& Finnegan, L. (1990). Patterns of interaction among drug dependent women and their infants. Pediatric Research, 24, 10A.

Frank, D.A., Jacobs, R.R., Beeghly, M., Augustyn, M., Bellinger, D., Cabral, H. \& Heeren, T. (2002). Level of prenatal cocaine exposure and scores on the Bayley Scales of Infant 
Development: modifiying effects of caregiver, early intervention, and birth weight. Pediatrics, 110, 1143-1152.

Griffith, D.R., Azuma, S.D. \& Chasnoff, I.J. (1994). Three-year outcome of children exposed prenatally to drugs. Journal of the American Academy of Child and Adolescence Psychiatry, $33,20-27$.

Gsellhofer, B., Fahrner, E.-M. \& Platt, J. (1994). European Addiction Severity Index (deutsche Fassung). München: Institut für Therapieforschung (IFT).

Hans, S.L. (2002). Studies of prenatal exposure to drugs focusing on parental care of children. Neurotoxicology \& Teratology, 24, 329-337.

Hans, S.L. \& Jeremy, R.J. (2001). Postneonatal mental and motor development of infants exposed in utero to opioid drugs. Infant Mental Health Journal, 22, 300-315.

Hogan, D.M. (1998). Annotation: the psychological development and welfare of children of opiate and cocaine users: Review and research needs. Journal of Child Psychology and Psychiatry, 39, 609-620.

Howard, J., Beckwith, L., Espinosa, M. \& Tyler, R. (1995). Development of infants born to cocaine-abusing women: biologic/maternal influences. Neurotoxicology and Teratology, 17, 403-411.

Horner, B.R. \& Scheibe, K.E. (1997). Prevalence and implications of Attention-Deficit Hyperactivity Disorder among adolescents in treatment for substance abuse. Journal of the American Academy of Adolescence Psychiatry, 36, 30-36.

Huestis, M.A. \& Choo, R.E. (2002). Drug abuse's smallest victims: in utero drug exposure. Forensic Science International, 128, 20-30.

Hurt, H., Malmud, E., Betancourt, L., Braitman, L.E., Brodsky, N.L. \& Giannetta, J. (1997). Children with in utero cocaine exposure do not differ from control subjects on intelligence testing. Archives of Pediatrics \& Adolescent Medicine, 151, 1237-1241.

Jacobson, S.W. \& Jacobson, J.L. (2001). Alcohol and drug-related effects on development: a new emphasis on contextual factors. Infant Mental Health Journal, 22, 416-430.

Jeremy, R.J. \& Bernstein, V.J. (1984). Dyads at risk: Methadone-maintained women and their 4-month-old-infants. Child Development, 55, 1141-1154. 
Johnson DE (2002). Adoption and the effect on children's development. Early Human Development, 68, 39-54.

Kaltenbach, K.A. (1996). Exposure to opiates: Behavioural outcomes in preschool and school-age children. NIDA Research Monographs, 164, 230-241.

Kaltenbach K.A., \& Finnegan L.P. (1987). Perinatal and developmental outcome of infants exposed to methadone in-utero. Neurotoxicology and Teratology, 9, 311-313.

Kandall, S.R., Gaines, J., Habel, L., Davidson, G. \& Jessop, D. (2000). Relationship of maternal substance abuse to subsequent sudden infant death syndrome in offspring: Journal of Pediatrics, 123, 120-126.

Kandel, D.B. (1984). Marijuana users in young adulthood. Archives of General Psychiatry, 41, 200-209.

Kandel, D.B. (1990). Parenting styles, drug use, and children's adjustment in families of young adults. Journal of Marriage and the Family, 52, 183-196

Kandel, D.B, Murphy, D. \& Karus, D. (1985). Cocaine use in young adulthood: Patterns of use and psychosocial correlates. In Kozel, N. und Adams, A. (Hrsg.). Cocaine use in America: Epidemiologic and clinical perspectives. Rockville, MD: National Institute on Drug Abuse.

Kelley, S.J, (2003). Cumulative environmental risk in substance abusing women: early intervention, parenting stress, child abuse potential and child development. Child Abuse \& Neglect, 27, 993-995.

Kienberger Jaudes, P., Ekwo, E. \& van Voorhis, J. (1995). Association of drug abuse and child abuse. Child Abuse \& Neglect, 19, 1065-1075.

Klein, M. (2001). Lebensqualität der Kinder von Opiatabhängigen: Fiktion, Tabu und Realität. In: Westerman, B., Jellinek, C. \& Bellmann, G.U. (Hrsg.). Substitution: Zwischen Leben und Sterben. Weinheim: Deutscher Studienverlag, S. 61-80.

Klein, M. (2002). Die besondere Gefährdung für Kinder aus Suchtfamilien - Präventive Ansätze. In: Gesamtverband für Suchtkrankenhilfe im Diakonischen Werk der EKD (Hrsg.). Handbuch für die Suchtkrankenhilfe. Wuppertal: Blaukreuz. Kapitel 7.2.5, S. 1-6.

Klein, M. (2003). Kinder drogenabhängiger Eltern. Fakten, Hintergründe, Perspektiven. Report Psychologie, 6, 358-371. 
Kochanska, G. (2001). Emotional development in children with different attachment histories: The first three years, Child Development, 72, 474-490.

Koren, G., Nulman, I., Rovet, J., Greenbaum, R.,. Loebstein, M. \& Einarson, T. (1998). Longterm neurodevelopmental risks in children exposed in utero to cocaine. In: Harvey, J.A. \& Kosofsky, B.E. (Hrsg): Cocaine. Effects on the developing brain (S 306-313) (Annals of the NYAS Vol 846). New York, NY: The New York Academy of Sciences.

Martin, C.S., Earleywine, M., Blackson, T.C., Vanyukow, M.M., Moss, H.B. \& Tarter, R.E. (1994). Aggressivity, Inattention, Hyperactivity, and Impulsivity in boys at high and low risk for substance abuse. Journal of Abnormal Child Psychology, 22, 177-203.

Mayes, L.C. (1996). Exposure to cocaine: Behavioural outcomes in preschool and school-age children. NIDA Research Monographs, 164, 211-229.

Mayes, L.C. (2003). Genetics of Childhood Disorders: LV. Prenatal Drug Exposure. Journal of the American Academy of Child and Adolescent Psychiatry, 42, 1258-1261.

Mayes, L.C., Cicchetti, D., Acharyya, S. \& Zhang, H. (2003). Developmental trajectories of cocaine- and-other-drug-exposed and non-cocaine-exposed children. Developmental and Behavioral Pediatrics, 24, 323-335.

Mayes, L.C., Crillon, C., Granger, R. \& Schottenfeld, R. (1998). Regulation of arousal and attention in preschool children exposed to cocaine prenatally. Annals of the New York Academy of Sciences, 846, 126-143.

Mayes, L.C., Feldman, R., Granger, R.H., Haynes, O.M., Bornstein, M.H. \& Schottenfeld, R. (1997). The effects of polydrug use with and without cocaine on mother-infant interacton at 3 and 6 months. Infant Behavior \& Development, 20, 489-502.

McGauhey, P.J., Starfield, B.; Alexander, C. \& Ensminger, M.E.(1991). Social environment and vulnerability of low birth weight children: A social-epidemiological perspective. Pediatrics, 88, 943-953.

Messinger, D.S., Bauer, C.R., Das, A., Seifer, R., Lester, B.M., Lagasse, L.L., Wright, L.L., Shankaran, S., Bada, H.S., Smeriglio, V.L., Langer, J.C., Beeghly, M. \& Poole, W.K. (2004). The maternal lifestyle study: cognitive, motor, and behavioral outcomes of cocaine-exposed and opiate-exposed infants through three years of age. Pediatrics, 113, 1677-1685. 
Moe, V. \& Slinning, K. (2001). Children prenatally exposed to substances: gender-related differences in outcome from infancy to 3 years of age. Infant Mental Health Journal, 22, 334350.

Moe, V. \& Slinning, K. (2002). Prenatal drug exposure and the conceptualiszation of longterm effects. Scandinavian Journal of Psychology, 43, 41-47.

Murphy, J.M, Jellinek, M., Quinn, D., Smith, G., Poitrast, F.G. \& Goshko, M. (1991). Substance abuse and serious child mistreatment: Prevalence, risk, and outcome in a court sample. Child Abuse \& Neglect, 15, 197-211.

Nair, P., Black, M.M., Schuler, M., Keane, V., Snow, L. \& Rigney, B.A. (1997). Risk factors for disruption in primary caregiving among infants of substance abusing women. Child Abuse \& Neglect, 21, 1039-1051.

Nair, P., Schuler, M.E., Black, M.M., Kettinger, L. \& Harrington, D. (2003). Cumulative environmental risk in substance abusing women: early intervention, parenting stress, child abuse potential and child development. Child Abuse \& Neglect, 27, 997-1017.

Nichtern, S. (1973). The children of drug users. Journal of the American Academy of Child Psychiatry, 12, 24-31.

Nulman, I., Rovet, J., Altmann, D., Bradley, C., Einarson, T. \& Koren, G. (1994). Neurodevelopment of adopted children exposed in utero to cocaine. Canadian Medical Association Journal, 151, 1591-1597.

Ornoy, A. \& Arnon, J. (1993). Clinical teratology. Western Journal of Medicine, 139, 382390.

Ornoy, A., Michailevskaya, V., Lukashov, I., Bar-Hamburger, R. \& Harel, S. (1996). The developmental outcomes of children born to heroin-dependent mothers, raised at home or adopted. Child Abuse \& Neglect, 20, 385-396.

Overmeyer, S., Schmidt, M.H. \& Blanz, B. (1993). Die Diagnostik belastender Lebensereignisse in der klinischen Routine mittels eines halbstandardisierten Interviews. Zeitschrift für Kinder- und Jugendpsychiatrie, 21, 5-13.

Perez-Bescos, L., Arrate-Zugazabeitia, J.K., Fortea-Jimeno, E., Echaniz-Urcelay, I, SaituaIturriaga, G. \& Delgado-Rubio, A. (1993). The clinical and epidemiological aspects of the neonatal abstinence syndrome. Boletin Medico Hospital Infantil de Mexico; 50, 843-848. 
Phelps, L., Wallace, N.V. \& Bontrager, A. (1997). Risk factors in early child development: is prenatal cocaine/polydrug exposure a key variable? Psychology in the Schools, 43, 245-252.

Poustka, F. (1993). Stand der Forschung zur 5. Achse der ICD 10 - Vergleich der Untersuchungen mit Unterstützung der WHO und der DFG. Zeitschrift für Kinder- und Jugendpsychiatrie und Psychotherapie, 21, Supplement 1. 44-44.

Poustka, F., Burk, B., Bästelein, M., van Goor-Lambo, G., Schermer, D. (1994). Elterninterview zur Achse V: Assoziierte aktuelle abnorme psychosoziale Umstände des Multiaxialen Klassifikationsschemas für psychiatrische Erkrankungen im Kindes- und Jugendalter (ICD.10) (Lifetime-Version) (nach dem Glossar des WHO-Dokuments: MNH/PRO/86.1 1.Rev. Genf, 1988, revidierte Fassung vom Aug. 1991/Nov. 1992). Frankfurt, Swets.

Powis, B., Gossop, M., Bury, C., Payne, K. \& Griffiths, P. (2000). Drug-using mothers: social, psychological and substance use problems of women opiate users with children. Drug and Alcohol Review, 19, 171-180.

Richardson, G.A. (1998). Prenatal cocaine exposure: A longitudinal study of development. Annals of the New York Academy of Sciences, 846, 144-152.

Richter, K.P. \& Bammer, G. (2000). A hierarchy of strategies heroin-using mothers employ to reduce harm to their children. Journal of Substance Abuse Treatment, 19, 403-413.

Rounsaville, B., Anton, S., Carrol, K., Budde, D., Prushoff, B. \& Gawin, F. (1991). Psychiatric diagnoses of treatment-seeking cocaine abusers. Archives of General Psychiatry $48,43-50$.

Rutter, M. (1987). Psychosocial resilience and protective mechanisms. American Journal of Orthopsychiatry, 57, 316-331.

Rutter, M. \& The ERA study team (1998). Developmental catch-up, and deficit, following adoption after severe global early privation. Journal of Child Psychology and Psychiatry, 39, 465-476.

Schneider, C., Fischer, G., Diamant, K., Hauk, R., Pezawas, L., Lenzinger, E. \& Kasper, S. (1996). Pregnancy and drug dependence. Wiener Klinische Wochenschrift, 108, 611 - 614.

Schulze, A. (Internet) http://bfg.rodiac.net/pdf/neonat._pflegetag/infos/Gefaehrdungen_des_ Kindes.pdf (3.3.2006) 
Shaffer, D., Gould, M.S, Brasic, P., Ambrosini, P., Fisher, P., Bird, H. \& Aluwahlia, S. (1983). A children’s global assessment scale (CGAS). Archives of General Psychiatry, 40, $1228-1231$

Simmel, C., Brooks, D., Barth, R.P. \& Hinshaw, S.P. (2001). Externalizing symptomatology among adoptive youth: Prevalence and preadoption risk factors. Journal of Abnormal Child Psychology, 29, 57-69.

Soeptami, S. (1994). Developmental outcomes of children of mothers dependent on heroin or heroin/methadone during pregnancy. Acta Paediatrica Supplement, 404, 36-39.

Tewes, U., Rossmann, P. \& Schallberger, U. (Hrsg.) (1999). Hamburg-WechslerIntelligenztest für Kinder (HAWIK-III). Huber, Bern.

Topitz, A., Ortner. R. \& Fischer, G. (2001). Substanzabhängigkeit in der Schwangerschaft eine Übersicht. In Riecher-Rössler, A. \& Rohde, A. (Hrsg.): Psychische Erkrankungen bei Frauen. Basel, Karger, S 220-226.

Tronick, E.Z. \& Beeghly, M. (1999). Prenatal cocaine exposure, child development, and the compromising effects of cumulative risk. Clinics in Perinatology, 26, 151-171

Tyler, R., Howard, J., Espinosa, M. \& Doakes, S.S. (1997). Placement with substance-abusing mothers vs. placement with other relatives: Infant outcomes. Child Abuse \& Neglect, 21, 337349.

Ukeje, I., Bendersky, M. \& Lewis, M. (2001). Mother-infant interaction at 12 months in prenatally cocaine-exposed children. American Journal of Drug and Alcohol Abuse, 27, 203224.

Urban, J., Carlson, E., Egeland, B. \& Sroufe, L.A. (1992). Patterns of individual adaptation across childhood. Development and Psychopathology, 3, 445-460.

Vaillant, G.E. \& Molfosky, E.S. (1982). The etiology of alcoholism: A prespective viewpoint. American Psychologist 37, 494-503.

Van Baar, A. \& de Graaff, B.M.T. (1994). Cognitive development at preschool-age of infants of drug-dependent mothers. Developmental Medicine and Child Neurology, 36, 1063-1075

Vathy, I. (2002). Prenatal opiate exposure: long-term CNS consequences in the stress system of the offspring. Psychoneuroendocrinology, 27, 273-283. 
Velez, M.L., Jansson, L.M., Montoya, I.D., Schweitzer, W., Golden, A. \& Svikis, D. (2004). Parenting knowledge among substance abusing women in treatment. Journal of Substance Abuse Treatment, 27, 215-222.

Walsh, C., MacMillan, H.L. \& Jamieson, E. (2003). The relationship between parental substance abuse and child maltreatment: Findings from the Ontario Health Supplement. Child Abuse \& Neglect, 27, 1409-1425.

Warren, S.B. (1992). Lower threshold for referral for psychiatric treatment for adopted adolescents. Journal of the American Academy of Child and Adolescent Psychiatry, 31, 512527.

Wilens, T.E., Biederman, J., Kiely, K., Bredin, E. \& Spencer, T.J. (1995). Pilot study of behavioral and emotional disturbances in the high-risk children of parents with opioid dependence. Journal of the American Academy of Child and Adolescence Psychiatry, 34, 779785 .

Wilson, G.S., McGreary, R., Kean, J. \& Baxter, J.C. (1979). The development of preschool children of heroin-addicted mothers: a controlled study. Pediatrics, 63, 135-141.

Yarrow, L.J. (1964). Separation from parents in early childhood. In M.L. Hoffman \& L. W. Hoffman (Hrsg.). Review of child development research (Vol. 1) (S 122). Chicago, IL: University of Chicago Press.

Ziegler, M. (1998). Biologische und psychosoziale Risikofaktoren für spätere kinder- und jugendpsychiatrische Auffälligkeiten bei Kindern mit neonatalem Abstinenzsyndrom. Med. Diss., Universität Frankfurt/M.

Ziegler, M., Poustka, F., v. Loewenich, V. \& Englert, E. (2000). Postpartale Risikofaktoren in der Entwicklung von Kindern opiatabhängiger Mütter. Nervenarzt, 71, 730-736. 
14 ANHANG 


\section{Anhang A - Gruppeneinteilung nach dem Betreuungsstatus}

\section{EG1 (Eltern):}

J1001: lebt bei Mutter und Stiefvater, keine Betreuungswechsel J1003: lebt bei Vater und Oma, Trennung der Eltern als 5 Monate J1007: lebt bei Mutter alleine

K1001: lebt bei Mutter und Stiefvater K1002: lebt bei Mutter und neuem Partner K1003: lebt bei beiden leiblichen Eltern K1005: lebt bei beiden leiblichen Eltern K1008: lebt bei beiden leiblichen Eltern K1014: lebt bei Mutter K1016: lebt bei beiden leiblichen Eltern K1017: lebt bei Mutter, viel Kontakt zu Vater K1018: lebt bei beiden leiblichen Eltern K1020: lebt bei beiden leiblichen Eltern K1023: lebt bei beiden leiblichen Eltern K1036: lebt bei beiden leiblichen Eltern K1045: lebt bei Vater und neuer Partnerin K1046: lebt bei beiden leiblichen Eltern J1028: lebt bei Mutter

\section{EG2 (Fremdbetreuung zu einem frühen Zeitpunkt)}

J1002: zu Adoptiveltern mit 3 Monaten, keine weiteren Betreuungswechsel (BW) J1004: zu Großmutter entlassen, keine weiteren Betreuungswechsel J1006: mit 12 Monaten von Eltern zu Großeltern, mit 11 Jahren wieder zu Vater zurück J1009: mit 6 Monaten von Mutter zu Großmutter, keine weiteren BW J1012: mit 3 Monaten von Mutter-Kind-Heim zu Pflegeeltern, keine weiteren BW J1014: mit 4 Monaten von Mutter-Kind-Heim zu Pflegeeltern, keine weiteren BW J1016: mit 6 Monaten von Eltern zu Großeltern, keine weiteren BW J1018: mit 6 Monaten ging Mutter weg, Kind blieb bei Großeltern, keine weitern BW J1021: mit 8 Monaten von Mutter zu Oma, keine weitern BW K1006: mit 18 Monaten ging Mutter von Großeltern weg, Kind blieb dort, keine weiteren BW K1024: Kind wird überwiegend von Großeltern aufgezogen, Mutter ist sporadisch da K1025: mit 3 Monaten zu Pflegeeltern, keine weiteren BW K1027: mit 3 Wochen zu Adoptiveltern, keine weiteren BW K1032: mit 6 Wochen zu Adoptiveltern, keine weiteren BW K1033: mit 4 Wochen zu Adoptiveltern, keine weiteren BW K1034: mit 6 Monaten von Pflegestelle zu Adoptiveltern, keine weiteren BW K1039: mit 2 Monaten zu Großmutter und Stiefgroßvater, keine weitern BW K1040: mit 9 Monaten von Mutter zu Großmutter und Stiefgroßvater, keine weiteren BW K1043: mit 1 Monat zu Adoptiveltern, keine weiteren BW 


\section{EG3 (Fremdbetreuung zu einem späten Zeitpunkt)}

J1013: mit 3,5 Jahren in Bereitschaftspflege, mit 4 Jahren zu Pflegeeltern, 2 BW

J1015: mit 4 Monaten von Mutter zu Pflegefamilie, mit 6 Monaten zu Tante, mit 2 Jahren zu Mutter, danach noch mal Wohngruppenaufenthalt, $5 \mathrm{BW}$

J1019: entlassen ins Heim, mit 9 Monaten zu Pflegefamilie, mit 5 Jahren KJP, mit 6 Jahren Übergangspflege, mit 6,5 Adoptivfamilie, 4 BW

K1013: mit 4 Jahren von Mutter zu Großmutter, keine weiteren BW

K1021: mit 8 Jahren von Mutter zu Großmutter, keine weiteren BW

K1026: mit 3 Jahren für 3 Monate zum getrennt lebenden Vater (Mutter Gefängnis), mit 5 Jahren von Mutter zu Pflegefamilie, 3 BW

K1028:langer Heimaufenthalt, mit 6 Monaten zu Eltern, wieder ins Heim, mit 15 Monaten zu Pflegeeltern, 3 BW

K1029: s.o.

K1030: mit 1 Jahr von Eltern zu Oma, mit 2 Jahren zu Pflegeeltern, 2 BW

K1031: mit 6 Monaten von Eltern zu Oma, mit 5 Jahren von Oma zu Pflegeeltern, 2 BW

K1037: mit 2 Jahren von Eltern zu Pflegeeltern, keine weiteren BW

K1038: mit 18 Monaten von Mutter zu Großvater, mit 19 Monaten zu Verwandten, mit knapp 8 zu Übergangspflege, mit 8 zu Pflegeltern, 4 BW

K1041: mit 21 Monaten von Eltern zu Oma, mit 3 Jahren zurück zu Eltern, mit 4 Jahren ins Kinderheim, 4 Monate später zu Pflegeeltern, 4 BW

K1042: mit 12 Monaten von Mutter ins Kinderheim, mit 18 Monaten zu Adoptiveltern, 2 BW

$$
\mathrm{N}=14
$$

\section{KG1 (Kontrollgruppe Eltern)}

J1005: lebt bei Eltern

J1008: lebt bei Mutter alleine

J1010: lebt bei Eltern

J1011: lebt bei Eltern

J1020: lebt bei Eltern

J1022: lebt bei Mutter alleine

K1004: lebt bei Eltern

K1007: lebt bei Mutter alleine

K1009: lebt bei Mutter alleine

K1010: lebt bei Vater und Großmutter, Mutter ging weg als 4 Jahre

K1012: lebt bei Eltern

K1015: lebt bei Eltern

K1022: lebt bei Mutter alleine

K1035: lebt bei Eltern

K1044: lebt bei Mutter alleine

J1025: lebt bei Vater

J1026: lebt bei Eltern 


\section{Anhang B - Achse VI: Stufen der Ausprägung}

0 Herausragende/gute soziale Funktionen: Herausragende/gute soziale Funktionen in allen sozialen Bereichen. Gute zwischenmenschliche Beziehungen mit Familie, Gleichaltrigen und Erwachsenen außerhalb der Familie; kann sich mit allen üblichen sozialen Situationen effektiv auseinandersetzen und verfügt über ein gutes Spektrum an Freizeitaktivitäten und Interessen.

1 Mäßige soziale Funktion: Insgesamt mäßige soziale Funktion, aber mit vorübergehenden oder geringeren Schwierigkeiten in nur ein oder zwei Bereichen (das Funktionsniveau kann - aber muss nicht - in ein oder zwei anderen Bereichen hervorragend sein).

2 Leichte soziale Beeinträchtigung: Adäquates Funktionsniveau in den meisten Bereichen aber leichte Schwierigkeiten in mindestens ein oder zwei Bereichen (wie z.B. Schwierigkeiten mit Freundschaften, gehemmte soziale Aktivitäten/Interessen, Schwierigkeiten mit innerfamiliären Beziehungen, wenig effektive soziale Coping-Mechanismen oder Schwierigkeiten in den Beziehungen zu Erwachsenen außerhalb der Familie).

3 Mäßige soziale Beeinträchtigung: Mäßige Beeinträchtigung in mindestens ein oder zwei Bereichen.

4 Ernsthafte soziale Beeinträchtigung: Ernsthafte Beeinträchtigung in mindestens ein oder zwei Bereichen (wie z.B. erheblicher Mangel an Freunden, Unfähigkeit, mit neuen sozialen Situationen zurecht zu kommen oder Schulbesuch nicht mehr möglich).

5 Ernsthafte und durchgängige soziale Beeinträchtigung: Ernsthafte Beeinträchtigung in den meisten Bereichen.

6 Funktionsunfähig in den meisten Bereichen: Benötigt ständige Aufsicht oder Betreuung zur basalen Alltagsbewältigung, ist nicht in der Lage, für sich selbst zu sorgen.

7 Schwere und durchgängige soziale Beeinträchtigung: manchmal unfähig für eine minimale Körperhygiene $\mathrm{zu}$ sorgen, oder braucht zeitweise strenge Beaufsichtigung um Gefahrensituationen für sich selbst oder andere zu verhüten, oder schwere Beeinträchtigung in allen Bereichen der Kommunikation.

8 Tiefe und durchgängige soziale Beeinträchtigung: Ständige Unfähigkeit für die eigenen Körperhygiene zu sorgen, oder ständige Gefahr, sich selbst oder Andere zu verletzen oder völliges Fehlen von Kommunikation.

$9 \quad$ Nicht zutreffend/nicht einschätzbar 


\section{Anhang C - Danksagung}

\section{Danken möchte ich...}

Herrn Prof. Dr. med. F. Poustka für die Ermöglichung dieser Promotion und sein stetiges Interesse am Fortgang dieser Arbeit.

Herrn Priv.-Doz. Dr. S. Bölte für die wertvolle fachliche Betreuung und abschließende Korrektur.

Frau Dipl.-Psych. P. Lang, die mir dieses Themas nach anfänglicher eigener Bearbeitung überlassen hat und von deren bis dahin erfolgter Bearbeitung ich profitieren konnte.

Tanja Kock für ihre Unterstützung im Rahmen ihrer Tätigkeit als wissenschaftliche Hilfskraft.

Den vielen hilfreichen Personen und Institutionen, durch die das Auffinden vieler Untersuchungsteilnehmer erst möglich wurde: Die Frankfurter Sozialrathäuser sowie der Besondere Dienst Jugendhilfe, stellvertretend für viele seien Frau Mikuszeit und Herr ReuterSpanier genannt, Frau Schwarz vom „Projekt Lichtblick“ der Integrativen Drogenhilfe, Frau Schutz von der Familienkasse der Bundesanstalt für Arbeit Hessen, OA Dr. Schlösser von der Station 32.7 der Neonatologie und Frau Dr. Domek.

Meinen Eltern, die mich trotz großer räumlicher Entfernung auf vielfältige Weise unterstützen.

Vor allem meinem Mann und meinen Kindern, die mich sowohl moralisch als auch praktisch unterstützt haben und auf Grund vieler „Arbeitswochenenden“ immer wieder zurückstehen mussten. 


\section{Anhang D - Curriculum vitae}

\section{Persönliche Daten}

geboren am

Geburtsname

Geburtsort

Familienstand

Kinder

Staatsangehörigkeit

Anschrift

$9 / 79-6 / 83$

$9 / 83-5 / 91$

$5 / 91$

WS 92/93 - SS 93

WS 93/94

$10 / 95$

$10 / 98$

$4 / 2000$

$11 / 2000$

seit $5 / 2001$

$8 / 91-9 / 92$

$8 / 96-6 / 97$
22.5.1973

Bachleitner

Wien

verheiratet

Tochter Anna Kristin (geb. am 11.12.1997)

Sohn Ben Elias (geb. am 24.07.2004)

Österreichisch

Am Rödergraben 8, 64342 Seeheim-Jugenheim

\section{Schulausbildung}

Grundschule Wien X

Bundesrealgymnasium Wien IV

Matura (Abitur)

\section{Studium}

Architekturstudium an der Technischen Universität Wien

Beginn des Diplomstudiums Psychologie an der Universität Wien Erste Diplomprüfung (Vordiplom)

Beginn der Diplomarbeit im Fachbereich Psychologische Diagnostik an der Universität Wien unter der Leitung von Prof. Dr.

K. Kubinger über das Thema „Psychologische Diagnostik bei Simulationsverdacht"

Zweite Diplomprüfung mit Auszeichnung

\section{Beruf}

Hospitanz an der Klinik für Psychiatrie und Psychotherapie des Kindes- und Jugendalters, Uniklinik Frankfurt/Main (EUStipendium)

Mitarbeiterin der Klinik für Psychiatrie und Psychotherapie des Kindes- und Jugendalters, Uniklinik Frankfurt/Main

\section{Auslandsaufenthalte}

Au-pair Aufenthalt in New Jersey (USA)

Studienaufenthalt in den USA an der University of California, Santa Cruz, durch das Joint-Study-Program der Universität Wien 


\section{Anhang E - Ehrenwörtliche Erklärung}

Ich erkläre, dass ich die dem Fachbereich Medizin der Johann Wolfgang Goethe-Universität Frankfurt am Main zur Promotionsprüfung eingereichte Dissertation mit dem Titel

\section{„Kognitive und emotionale Entwicklung von Kindern mit pränataler Opiatexposition in} Abhängigkeit der postnatalen Betreuungsbedingungen““

im Zentrum der Psychiatrie, Klinik für Psychiatrie und Psychotherapie des Kindes- und Jugendalters des Klinikums der Johann Wolfgang Goethe-Universität Frankfurt am Main unter Betreuung und Anleitung von Prof. Dr. med. F. Poustka und mit Unterstützung von Priv.- Doz. Dr. S. Bölte ohne sonstige Hilfe selbst durchgeführt und bei der Abfassung der Arbeit keine anderen als die in der Dissertation angeführten Hilfsmittel benutzt habe.

Ich habe bisher an keiner in- oder ausländischen Universität ein Gesuch um Zulassung zur Promotion eingereicht, noch die vorliegende Arbeit als Dissertation vorgelegt.

Frankfurt am Main, 5. April 2007

Silke Schiemann 